\title{
Design concepts for the Cherenkov Telescope Array CTA: an advanced facility for ground-based high-energy gamma-ray astronomy
}

\author{
The CTA Consortium
}

Received: 26 November 2010 / Accepted: 21 July 2011 / Published online: 23 November 2011 (C) The Author(s) 2011. This article is published with open access at Springerlink.com

\begin{abstract}
Ground-based gamma-ray astronomy has had a major breakthrough with the impressive results obtained using systems of imaging atmospheric Cherenkov telescopes. Ground-based gamma-ray astronomy has a huge potential in astrophysics, particle physics and cosmology. CTA is an international initiative to build the next generation instrument, with a factor of 5-10 improvement in sensitivity in the $100 \mathrm{GeV}-10 \mathrm{TeV}$ range and the extension to energies well below $100 \mathrm{GeV}$ and above $100 \mathrm{TeV}$. CTA will consist of two arrays (one in the north, one in the south) for full sky coverage and will be operated as open observatory. The design of CTA is based on currently available technology. This document reports on the status and presents the major design concepts of CTA.
\end{abstract}

Keywords Ground based gamma ray astronomy •

Next generation Cherenkov telescopes $\cdot$ Design concepts

\section{Executive summary}

The present generation of imaging atmospheric Cherenkov telescopes (H.E.S.S., MAGIC and VERITAS) has in recent years opened the realm of

Contact: W. Hofmann (Werner.Hofmann@mpi-hd.mpg.de), M. Martínez (martinez@ifae.es),

CTA Project Office, Landessternwarte, Universität Heidelberg, Königstuhl,

69117 Heidelberg, Germany.

The full author list and affiliations are given at the end of the paper.

The CTA Consortium

URL: http://www.cta-observatory.org

e-mail: J.Knapp@leeds.ac.uk 
ground-based gamma ray astronomy for energies above a few tens of $\mathrm{GeV}$. The Cherenkov Telescope Array (CTA) will explore in depth our Universe in very high energy gamma-rays and investigate cosmic processes leading to relativistic particles, in close cooperation with observatories of other wavelength ranges of the electromagnetic spectrum, and those using cosmic rays and neutrinos.

Besides guaranteed high-energy astrophysics results, CTA will have a large discovery potential in key areas of astronomy, astrophysics and fundamental physics research. These include the study of the origin of cosmic rays and their impact on the constituents of the Universe through the investigation of galactic particle accelerators, the exploration of the nature and variety of black hole particle accelerators through the study of the production and propagation of extragalactic gamma rays, and the examination of the ultimate nature of matter and of physics beyond the Standard Model through searches for dark matter and the effects of quantum gravity.

With the joining of the US groups of the Advanced Gamma-ray Imaging System (AGIS) project, and of the Brazilian and Indian groups in Spring 2010, and with the strong Japanese participation, CTA represents a genuinely worldwide effort, extending well beyond its European roots.

CTA will consist of two arrays of Cherenkov telescopes, which aim to: (a) increase sensitivity by another order of magnitude for deep observations around $1 \mathrm{TeV}$, (b) boost significantly the detection area and hence detection rates, particularly important for transient phenomena and at the highest energies, (c) increase the angular resolution and hence the ability to resolve the morphology of extended sources, (d) provide uniform energy coverage for photons from some tens of $\mathrm{GeV}$ to beyond $100 \mathrm{TeV}$, and (e) enhance the sky survey capability, monitoring capability and flexibility of operation. CTA will be operated as a proposal-driven open observatory, with a Science Data Centre providing transparent access to data, analysis tools and user training.

To view the whole sky, two CTA sites are foreseen. The main site will be in the southern hemisphere, given the wealth of sources in the central region of our Galaxy and the richness of their morphological features. A second complementary northern site will be primarily devoted to the study of Active Galactic Nuclei (AGN) and cosmological galaxy and star formation and evolution. The performance and scientific potential of arrays of Cherenkov telescopes have been studied in significant detail, showing that the performance goals can be reached. What remains to be decided is the exact layout of the telescope array. Ample experience exists in constructing and operating telescopes of the 12-m class (H.E.S.S., VERITAS). Telescopes of the 17-m class are operating (MAGIC) and one 28-m class telescope is under construction (H.E.S.S. II). These telescopes will serve as prototypes for CTA. The structural and optical properties of such telescopes are well understood, as many have been built for applications from radio astronomy to solar power installations. The fast electronics needed in gamma ray astronomy to capture the nanosecond-scale Cherenkov pulses have long been mastered, well before such electronics became commonplace with the Gigahertz transmission and processing used today in telephony, internet, television, and computing. 
The extensive experience of members of the consortium in the area of conventional photomultiplier tubes (PMTs) provides a solid foundation for the design of cameras with an optimal cost/performance ratio. Consequently, the base-line design relies on conventional PMTs. Advanced photon detectors with improved quantum efficiency are under development and test and may well be available when the array is constructed. In short, all the technical solutions needed to carry out this project exist today. The main challenge lies in the industrialisation of all aspects of the production and the exploitation of economies of scale.

Given the large amounts of data recorded by the instrument and produced by computer simulations of the experiment, substantial efforts in e-science and grid computing are envisaged to enable efficient data processing. Some of the laboratories involved in CTA are Tier 1 and 2 centres on the LHC computing grid and the Cosmogrid. Simulation and analysis packages for CTA are developed for the grid. The consortium has set up a CTA-Virtual Organisation within the EGEE project (Enabling Grids for E-sciencE; funded by the European Union) for use of grid infrastructure and the sharing of computing resources, which will facilitate worldwide collaboration for simulations and the processing and analysis of scientific data.

Unlike current ground-based gamma-ray instruments, CTA will be an open observatory, with a Science Data Centre (SDC) which provides preprocessed data to the user, as well as the tools necessary for the most common analyses. The software tools will provide an easy-to-use and well-defined access to data from this unique observatory. CTA data will be accessible through the Virtual Observatory, with varying interfaces matched to different levels of expertise. The required toolkit is being developed by partners with experience in SDC management from, for example, the INTEGRAL space mission.

Experiments in astroparticle physics have proven to be an excellent training ground for young scientists, providing a highly interdisciplinary work environment with ample opportunities to acquire not only physics skills but also to learn data processing and data mining techniques, programming of complex control and monitoring systems and design of electronics. Further, the environment of the large multi-national CTA Collaboration, working across international borders, ensures that presentation skills, communication ability and management and leadership proficiency are enhanced. Young scientists frequently participate in outreach activities and, thus, hone also their skills in this increasingly important area. With its training and mobility opportunities for young scientists, CTA will have a major impact on society.

Outreach activities will be an important part of the CTA operation. Lectures and demonstrations augmented by web-based non-expert tools for viewing CTA data will be offered to pupils and lay audiences. Particularly interesting objects will be featured on the CTA web pages, along the lines of the "Source of the Month" pages of the H.E.S.S. collaboration. CTA is expected to make highly visible contributions towards popularising science and generating 


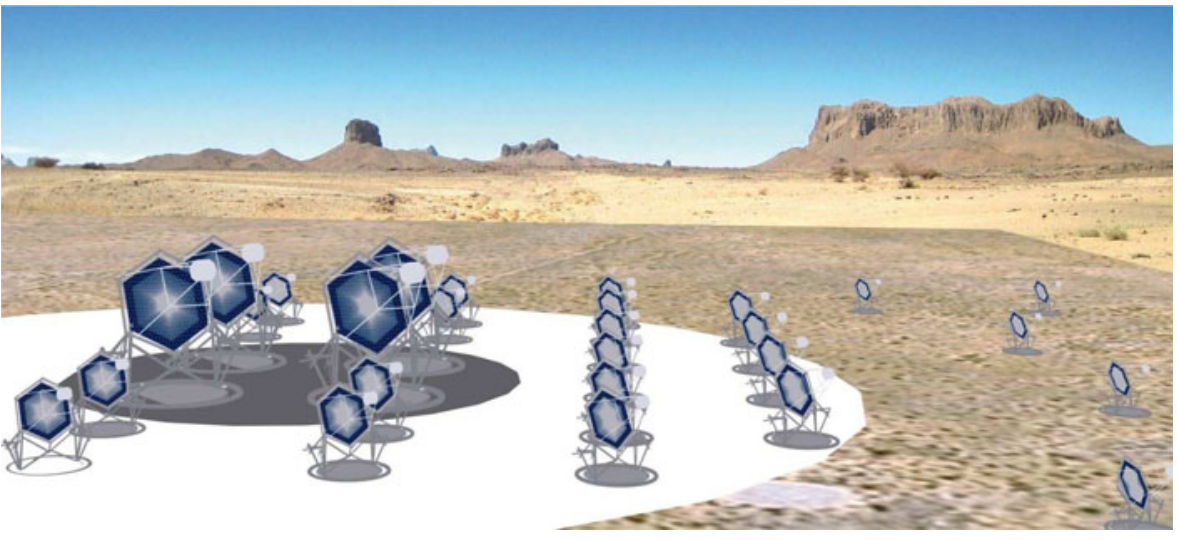

Fig. 1 Conceptual layout of a possible Cherenkov Telescope Array (not to scale)

enthusiasm for research at the cosmic frontier and to create interest in the technologies applied in this field.

\section{CTA, a new science infrastructure}

In the field of very high energy gamma-ray astronomy (VHE, energies $>100 \mathrm{GeV}^{1}$ ), the instruments H.E.S.S. (http://www.mpi-hd.mpg.de/hfm/ HESS), MAGIC (http://magic.mppmu.mpg.de) and VERITAS (http://veritas. sao.arizona.edu) have been driving the development in recent years. The spectacular astrophysics results from the current Cherenkov instruments have generated considerable interest in both the astrophysics and particle physics communities and have created the desire for a next-generation, more sensitive and more flexible facility, able to serve a larger community of users. The proposed $\mathrm{CTA}^{2}$ (http://www.cta-observatory.org) is a large array of Cherenkov telescopes of different sizes, based on proven technology and deployed on an unprecedented scale (Fig. 1). It will allow significant extension of our current knowledge in high-energy astrophysics. CTA is a new facility, with capabilities well beyond those of conceivable upgrades of existing instruments such as H.E.S.S., MAGIC or VERITAS. The CTA project unites the main research groups in this field in a common strategy, resulting in an unprecedented convergence of efforts, human resources, and know-how. Interest in and support for the project is coming from scientists in Europe, America, Asia and Africa, all of whom wish to use such a facility for their research and are willing to contribute to its design and construction. CTA will offer worldwide unique opportunities to users with varied scientific interests. The number of

\footnotetext{
${ }^{1} 1 \mathrm{GeV}=10^{9} \mathrm{eV} ; 1 \mathrm{TeV}=10^{12} \mathrm{eV} ; 1 \mathrm{PeV}=10^{15} \mathrm{eV}$.

${ }^{2}$ CTA was first publicly presented to an ESFRI panel in Autumn 2005.
} 
in particular young scientists working in the still evolving field of gamma-ray astronomy is growing at a steady rate, drawing from other fields such as nuclear and particle physics. In addition, there is increased interest by other parts of the astrophysical community, ranging from radio to X-ray and satellite-based gamma-ray astronomers. CTA will, for the first time in this field, provide open access via targeted observation proposals and generate large amounts of public data, accessible using Virtual Observatory tools. CTA aims to become a cornerstone in a networked multi-wavelength, multi-messenger exploration of the high-energy non-thermal universe.

\section{The science case for CTA}

\subsection{Science motivation in a nutshell}

\subsubsection{Why observing in gamma-rays?}

Radiation at gamma-ray energies differs fundamentally from that detected at lower energies and hence longer wavelengths: $\mathrm{GeV}$ to $\mathrm{TeV}$ gamma-rays cannot conceivably be generated by thermal emission from hot celestial objects. The energy of thermal radiation reflects the temperature of the emitting body, and apart from the Big Bang there is and has been nothing hot enough to emit such gamma-rays in the known Universe. Instead, we find that highenergy gamma-rays probe a non-thermal Universe, where other mechanisms allow the concentration of large amounts of energy onto a single quantum of radiation. In a bottom-up fashion, gamma-rays can be generated when highly relativistic particles-accelerated for example in the gigantic shock waves of stellar explosions - collide with ambient gas, or interact with photons and magnetic fields. The flux and energy spectrum of the gamma-rays reflects the flux and spectrum of the high-energy particles. They can therefore be used to trace these cosmic rays and electrons in distant regions of our own Galaxy or even in other galaxies. High-energy gamma-rays can also be produced in a top-down fashion by decays of heavy particles such as hypothetical dark matter particles or cosmic strings, both of which might be relics of the Big Bang. Gamma-rays therefore provide a window on the discovery of the nature and constituents of dark matter.

High-energy gamma-rays, as argued above, can be used to trace the populations of high-energy particles in distant regions of our own or in other galaxies. Meandering in interstellar magnetic fields, cosmic rays will usually not reach Earth and thus cannot be observed directly. Those which do arrive have lost all directional information and cannot be used to pinpoint their sources, except for cosmic-rays of extreme energy $>10^{18} \mathrm{eV}$. However, such high-energy particle populations are an important aspect of the dynamics of galaxies. Typically, the energy content in cosmic rays equals the energies in magnetic fields or in thermal radiation. The pressure generated by high-energy particles drives galactic outflows and helps balance the gravitational collapse 
of galactic disks. Astronomy with high-energy gamma-rays is so far the only way to directly probe and image the cosmic particle accelerators responsible for these particle populations, in conjunction with studies of the synchrotron radiation resulting form relativistic electrons moving in magnetic fields and giving rise to non-thermal radio and $\mathrm{X}$-ray emission.

\subsubsection{A first glimpse of the astrophysical sources of gamma-rays}

The first images of the Milky Way in VHE gamma-rays have been obtained in the last few years. These reveal a chain of gamma-ray emitters situated along the Galactic equator (see Fig. 2), demonstrating that sources of highenergy radiation are ubiquitous in our Galaxy. Sources of this radiation include supernova shock waves, where presumably atomic nuclei are accelerated and generate the observed gamma-rays. Another important class of objects are "nebulae" surrounding pulsars, where giant rotating magnetic fields give rise to a steady flow of high-energy particles. Additionally, some of the objects discovered to emit at such energies are binary systems, where a black hole or a pulsar orbits a massive star. Along the elliptical orbit, the conditions for particle acceleration vary and hence the intensity of the radiation is modulated with the orbital period. These systems are particularly interesting in that they enable the study of how particle acceleration processes respond
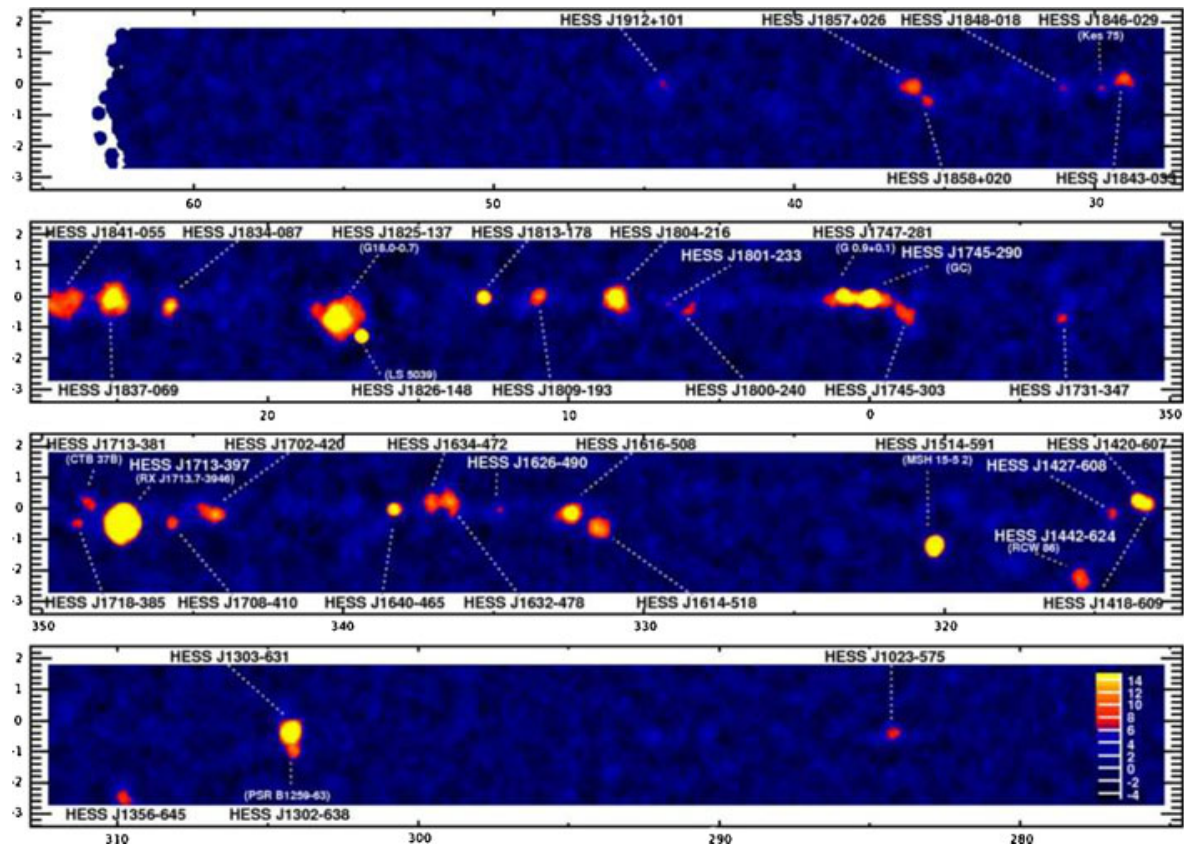

Fig. 2 The Milky Way viewed in VHE gamma-rays, in four bands of Galactic longitude [1] 
to varying ambient conditions. One of several surprises was the discovery of "dark sources", objects which emit VHE gamma rays, but have no obvious counterpart in other wavelength regimes. In other words, there are objects in the Galaxy which might in fact be only detectable in high-energy gamma-rays. Beyond our Galaxy, many extragalactic sources of high-energy radiation have been discovered, located in active galaxies, where a super-massive black hole at the centre of the galaxy is fed by a steady stream of gas and is releasing enormous amounts of energy. Gamma-rays are believed to be emitted from the vicinity of these black holes, allowing the study of the processes occurring in this violent and as yet poorly understood environment.

\subsubsection{Cherenkov telescopes}

The recent breakthroughs in VHE gamma-ray astronomy were achieved with ground-based Cherenkov telescopes. When a VHE gamma-ray enters the atmosphere, it interacts with atmospheric nuclei and generates a shower of secondary electrons, positrons and photons. Moving through the atmosphere at speeds higher than the speed of light in air, these electrons and positrons emit a beam of bluish light, the Cherenkov light. For near vertical showers this Cherenkov light illuminates a circle with a diameter of about $250 \mathrm{~m}$ on the ground. For large zenith angles the area can increase considerably. This light can be captured with optical elements and be used to image the shower, which vaguely resembles a shooting star. Reconstructing the shower axis in space and tracing it back onto the sky allows the celestial origin of the gamma-ray to be determined. Measuring many gamma-rays enables an image of the gamma-ray sky, such as that shown in Fig. 2, to be created. Large optical reflectors with areas in the $100 \mathrm{~m}^{2}$ range and beyond are required to collect enough light, and the instruments can only be operated in dark nights at clear sites. With Cherenkov telescopes, the effective area of the detector is about the size of the Cherenkov pool at ground. As this is a circle with 250-m diameter this is about $10^{5} \times$ larger than the size that can be achieved with satellite-based detectors. Therefore much lower fluxes at higher energies can be investigated with Cherenkov Telescopes, enabling the study of short time scale variability.

The Imaging Atmospheric Cherenkov Technique was pioneered by the Whipple Collaboration in the United States. After more than 20 years of development, the Crab Nebula, the first source of VHE gamma-rays, was discovered in 1989. The Crab Nebula is among the strongest sources of very high energy gamma-rays, and is often used as a "standard candle". Modern instruments, using multiple telescopes to track the cascades from different perspectives and employing fine-grained photon detectors for improved imaging, can detect sources down to $1 \%$ of the flux of the Crab Nebula. Finelypixellated imaging was first employed in the French CAT telescope [2], and the use of "stereoscopic" telescope systems to provide images of the cascade from different viewing points was pioneered by the European HEGRA IACT system [3]. For summaries of the achievements in recent years and the science case for a next-generation very high energy gamma ray observatory see [4-8]. 
In March 2007, the High Energy Stereoscopic System (H.E.S.S.) project was awarded the Descartes Research Prize of the European Commission for offering "A new glimpse at the highest-energy Universe". Together with the instruments MAGIC and VERITAS (in the northern hemisphere) and CANGAROO (in the southern hemisphere), a new wavelength domain was opened for astronomy, the domain of very high energy gamma-rays with energies between about $100 \mathrm{GeV}$ and about $100 \mathrm{TeV}$, energies which are a million million times higher than the energy of visible light.

At lower energies, in the $\mathrm{GeV}$ domain, the launch of a new generation of gamma-ray telescopes (like AGILE, but in particular Fermi, which was launched in 2008) has opened a new era in gamma-ray discoveries [9]. The Large Area Telescope (LAT), the main instrument onboard Fermi, is sensitive to gamma-rays with energies in the range from $20 \mathrm{MeV}$ to about $100 \mathrm{GeV}$. The energy range covered by CTA will smoothly connect to that of Fermi-LAT and overlap with that of the current generation of ground based instruments and extends to the higher energies, while providing an improvement in both sensitivity and angular resolution.

\subsection{The CTA science drivers}

The aims of the CTA can be roughly grouped into three main themes, serving as key science drivers:

1. Understanding the origin of cosmic rays and their role in the Universe

2. Understanding the nature and variety of particle acceleration around black holes

3. Searching for the ultimate nature of matter and physics beyond the Standard Model

Theme 1 comprises the study of the physics of galactic particle accelerators, such as pulsars and pulsar wind nebulae, supernova remnants, and gammaray binaries. It deals with the impact of the accelerated particles on their environment (via the emission from particle interactions with the interstellar medium and radiation fields), and the cumulative effects seen at various scales, from massive star forming regions to starburst galaxies.

Theme 2 concerns particle acceleration near super-massive and stellar-sized black holes. Objects of interest include microquasars at the Galactic scale, and blazars, radio galaxies and other classes of AGN that can potentially be studied in high-energy gamma rays. The fact that CTA will be able to detect a large number of these objects enables population studies which will be a major step forward in this area. Extragalactic background light (EBL), Galaxy clusters and Gamma Ray Burst (GRB) studies are also connected to this field.

Finally, Theme 3 covers what can be called "new physics", with searches for dark matter through possible annihilation signatures, tests of Lorentz invariance, and any other observational signatures that may challenge our current understanding of fundamental physics.

CTA will be able to generate significant advances in all these areas. 


\subsection{Details of the CTA science case}

We conclude this chapter with a few examples of physics issues that could be significantly advanced with an instrument like CTA. The list is certainly not exhaustive. The physics of the CTA is being explored in detail by many scientists and their findings indicate the huge potential for numerous interesting discoveries with CTA.

\subsubsection{Cosmic ray origin and acceleration}

A tenet of high-energy astrophysics is that cosmic rays (CRs) are accelerated in the shocks of supernova explosions. However, while particle acceleration up to energies well beyond $10^{14} \mathrm{eV}$ has now clearly been demonstrated with the current generation of instruments, it is by no means proven that supernovae accelerate the bulk of cosmic rays. The large sample of supernovae which will be observable with CTA - in some scenarios several hundreds of objectsand in particular the increased energy coverage at lower and higher energies, will allow sensitive tests of acceleration models and determination of their parameters. Improved angular resolution (arcmin) will help to resolve fine structures in supernova remnants which are essential for the study of particle acceleration and particle interactions. Pulsar wind nebulae surrounding the pulsars (created in supernova explosions) are another abundant source of high-energy particles, including possibly high-energy nuclei. Energy conversion within pulsar winds and the interaction of the wind with the ambient medium and the surrounding supernova shell challenge current ideas in plasma physics.

The CR spectrum observed near the Earth can be described by a pure power law up to an energy of a few $\mathrm{PeV}$, where it slightly steepens. The feature is called the "knee". The absence of other features in the spectrum suggests that, if supernova remnants (SNRs) are the sources of galactic CRs, they must be able to accelerate particles at least up to the knee. For this to happen, the acceleration in diffusive shocks has to be fast enough for particles to reach PeV energies before the SNR enters the Sedov phase, when the shock slows down and consequently becomes unable to confine the highest energy CRs [10] Since the initial free expansion velocity of SNRs does not vary much from object to object, only the amplification of magnetic fields can increase the acceleration rate to the required level. Amplification factors of 100-1,000 compared to the interstellar medium value and small diffusion coefficients are needed [11]. The non-linear theory of diffusive shock acceleration suggests that such an amplification of the magnetic field might be induced by the CRs themselves, and high resolution X-ray observations of SNR shocks seem to support this scenario, though their interpretation is debated. Thus, an accurate determination of the intensity of the magnetic field at the shock is of crucial importance for disentangling the origin of the observed gamma-ray emission and understanding the way diffusive shock acceleration works. 
Even if a SNR can be detected by Cherenkov telescopes during a significant fraction of its lifetime (up to several $10^{4}$ years), it can make $10^{15} \mathrm{eV}$ CRs only for a much shorter time (several hundred years), due to the rapid escape of $\mathrm{PeV}$ particles from the SNR. This implies that the number of SNRs which have currently a gamma-ray spectrum extending up to hundreds of $\mathrm{TeV}$ is very roughly of the order of $\sim 10$. The actual number of detectable objects will depend on the distance and on the density of the surrounding interstellar medium. The detection of such objects (even a few of them) would be extremely important, as it would be clear evidence for the acceleration of CRs up to PeV energies in SNRs. A sensitive scan of the galactic plane with CTA would be an ideal way of searching for these sources. In general, the spectra of radiating particles (both electrons and protons) and therefore also the spectra of gamma-ray radiation, should show characteristic curvature, reflecting acceleration at CR modified shocks. However, to see such curvature, one needs a coverage of a few decades in energy, far from the cutoff region. CTA will provide this coverage. If the general picture of SNR evolution described above is correct, the position of the cutoff in the gamma-ray spectrum depends on the age of the SNR and on the magnetic field at the shock. A study of the number of objects detected as a function of the cutoff energy will allow tests of this hypothesis and constraints to be placed on the physical parameters of SNRs, in particular of the magnetic field strength.

CTA offers the possibility of real breakthroughs in the understanding of cosmic rays; as there is the potential to directly observe their diffusion (see, e.g., [12]) The presence of a massive molecular cloud located in the proximity of a SNR (or any kind of CR accelerator) provides a thick target for CR hadronic interactions and thus enhances the gamma-ray emission. Hence, studies of molecular clouds in gamma-rays can be used to identify the sites where CRs are accelerated. While travelling from the accelerator to the target, the spectrum of cosmic rays is a strong function of time, distance to the source, and the (energy-dependent) diffusion coefficient. Depending on the values of these parameters varying proton, and therefore gamma-ray, spectra may be expected. CTA will allow the study of emission depending on these three quantities, which is impossible with current experiments. A determination, with high sensitivity, of spatially resolved gamma-ray sources related to the same accelerator would lead to the experimental determination of the local diffusion coefficient and/or the local injection spectrum of cosmic rays. Also, the observation of the penetration of cosmic rays into molecular clouds will be possible. If the diffusion coefficient inside a cloud is significantly smaller than the average in the neighbourhood, low energy cosmic rays cannot penetrate deep into the cloud, and part of the gamma-ray emission from the cloud is suppressed, with the consequence that its gamma-ray spectrum appears harder than the cosmic-ray spectrum.

Both of these effects are more pronounced in the denser central region of the cloud. Thus, with an angular resolution of the order of $\leq 1$ arcmin one could resolve the inner part of the clouds and measure the degree of penetration of cosmic rays [13]. 
More information on general aspects of cosmic rays and their relationship to VHE gamma observations is available in the review talks and papers presented at the International Cosmic Ray Conference 2009 held in Łódź and the online proceedings are a good source of information [14].

\subsubsection{Pulsar wind nebulae}

Pulsar wind nebulae (PWNe) currently constitute the most populous class of identified Galactic VHE gamma-ray sources. As is well known, the Crab Nebula is a very effective accelerator (shown by emission across more than 15 decades in energy) but not an effective inverse Compton gamma-ray emitter. Indeed, we see gamma rays from the Crab because of its large spin-down power $\left(\sim 10^{38} \mathrm{erg} \mathrm{s}^{-1}\right)$, although the gamma-ray luminosity is much less than the spin-down power of its pulsar. This can be understood as resulting from a large $(\mathrm{mG})$ magnetic field, which also depends on the spin-down power. A less powerful pulsar would imply a weaker magnetic field, which would allow a higher gamma-ray efficiency (i.e. a more efficient sharing between synchrotron and inverse Compton losses). For instance, HESS J1825-137 has a similar TeV luminosity to the Crab, but a spin-down power that is 2 orders of magnitude smaller, and its magnetic field has been constrained to be in the range of a few, instead of hundreds, of $\mu \mathrm{G}$. The differential gamma-ray spectrum of the whole emission region from the latter object has been measured over more than two orders of magnitude, from $270 \mathrm{GeV}$ to $35 \mathrm{TeV}$, and shows indications of a deviation from a pure power law that CTA could confirm and investigate in detail. Spectra have also been determined for spatially separated regions of HESS J1825-137 [15]. Another example is HESS J1303-61 [16] The photon spectra in the different regions show a softening with increasing distance from the pulsar and therefore an energy dependent morphology. If the emission is due to the inverse Compton effect, the pulsar power is not sufficient to generate the gamma-ray luminosity, suggesting that the pulsar had a higher injection power in the past. Is this common for other PWNe and what can that tell us about the evolution of pulsar winds? In the case of Vela X [17], the first detection of what appears to be a VHE inverse Compton peak in the spectral energy distribution (SED) was found. Although a hadronic interpretation has also been put forward it is as yet unclear how large the contribution of ions to the pulsar wind could be. CTA can be used to test leptonic vs. hadronic models of gamma-ray production in PWNe.

The return current problem for pulsars have not been solved to date, but if we detect a clear hadronic signal, this will show that ions are extracted from the pulsar surface, which may lead to a solution of the most fundamental question in pulsar magnetospheric physics: how do we close the pulsar current? In systems where we see a clear leptonic signal, it is important to measure the magnetisation (or "sigma") parameter of the PWNe. Are the magnetic fields and particles in these systems in equipartition (as in the Crab Nebula) or do have particle dominated winds? This will contribute significantly to the understanding of the magnetohydrodynamic flow in PWNe. Understanding 
the time evolution of the multi-wavelength synchrotron and inverse Compton (or hadronic) intensities is also an aim of CTA. Such evolutionary tracks are determined by the nature of the progenitor stellar wind, the properties of the subsequent composite SNR explosion and the surrounding interstellar environment. Finally, the sensitivity and angular resolution achievable with CTA will allow detailed multi-wavelength studies of large/close PWNe, and the understanding of particle propagation, the magnetic field profile in the nebula, and inter-stellar medium (ISM) feedback.

The evolution and structure of pulsar wind nebulae is discussed in a recent review [18]. Many key implications for VHE gamma ray measurements, and an assessment of the current observations can be found in [19].

\subsubsection{The galactic centre region}

It is clear that the galactic centre region itself will be one of the prime science targets for the next generation of VHE instruments [20, 21]. The galactic centre hosts the nearest super-massive black hole, as well as a variety of other objects likely to generate high-energy radiation, including hypothetical darkmatter particles which may annihilate and produce gamma-rays. Indeed, the galactic centre has been detected as a source of high-energy gamma-rays, and indications for high-energy particles diffusing away from the central source and interacting with the dense gas clouds in the central region have been observed. In observations with improved sensitivity and resolution, the galactic centre can potentially yield a variety of interesting results on particle acceleration and gamma-ray production in the vicinity of black holes, on particle propagation in central molecular clouds, and, possibly, on the detection of dark matter annihilation or decay.

The VHE gamma-ray view of the galactic centre region is dominated by two point sources, one coincident with a PWN inside SNR G0.9+0.1, and one coincident with the super-massive black hole Sgr A* and another putative PWN (G359.95-0.04). After subtraction of these sources diffuse emission along the galactic centre ridge is visible, which shows two important features: it appears correlated with molecular clouds (as traced by the CS (1-0) line), and it exceeds by a factor of 3 to 9 the gamma-ray emission that would be produced if the same target material was exposed to the cosmic-ray environment in our local neighbourhood. The striking correlation of diffuse gamma-ray emission with the density of molecular clouds within $\sim 150 \mathrm{pc}$ of the galactic centre favours a scenario in which cosmic rays interact with the cloud material and produce gamma-rays via the decay of neutral pions. The differential gammaray flux is stronger and harder than expected from just "passive" exposure of the clouds to the average galactic cosmic ray flux, suggesting one or more nearby particle accelerators are present. In a first approach, the observed gamma-ray morphology can be explained by cosmic rays diffusing away from an accelerator near the galactic centre into the surroundings. Adopting a diffusion coefficient of $D=O\left(10^{30}\right) \mathrm{cm}^{2} / \mathrm{s}$, the lack of VHE gamma-ray emission beyond $150 \mathrm{pc}$ in this model points to an accelerator age of no more 
than $10^{4}$ years. Clearly, improved sensitivity and angular resolution would permit the study of the diffusion process in great detail, including any possible energy dependence. An alternative explanation (which CTA will address) is the putative existence of a number of electron sources (e.g. PWNe) along the galactic centre ridge, correlated with the density of molecular clouds. Given the complexity and density of the source population in the galactic centre region, CTA's improved sensitivity and angular resolution is needed to map the morphology of the diffuse emission, and to test its hadronic or leptonic origin.

CTA will also measure VHE absorption in the interstellar radiation field (ISRF). This is impossible for other experiments, like Fermi-LAT, as their energy coverage is too small, and very hard or perhaps impossible for current air Cherenkov experiments, as they lack the required sensitivity. At $8 \mathrm{kpc}$ distance, VHE gamma-ray attenuation due to the CMB is negligible for energies $<500 \mathrm{TeV}$. But the attenuation due to the ISRF (which has a comparable number density at wavelengths $20-300 \mu \mathrm{m}$ ) can produce absorption at about $50 \mathrm{TeV}$ [22]. Observation of the cutoff energy for different sources will provide independent tests and constraints of ISRF models. CTA will observe sources at different distances and thereby independently measure the absorption model and the ISRF. Due to their smaller distances there is less uncertainty in identifying intrinsic and extrinsic features in the spectrum than is the case for EBL studies.

\subsubsection{Microquasars, gamma-ray, and X-ray binaries}

Currently, a handful of VHE gamma-ray emitters are known to be binary systems, consisting of a compact object, a neutron star or a black hole, orbiting a massive star. Whilst many questions on the gamma-ray emission from such systems are still open (in some cases it is not even clear if the energy source is a pulsar-driven nebula around a neutron star or accretion onto a black hole) it is evident that they offer a unique chance to "experiment" with cosmic accelerators. Along the eccentric orbits of the compact objects, the environment (including the radiation field) changes, resulting in a periodic modulation of the gamma-ray emission, allowing the study of how particle acceleration is affected by environmental conditions. Interestingly, the physics of microquasars in our own Galaxy resembles the processes occurring around super-massive black holes in distant active galaxies, with the exception of the much reduced time scales, providing insights in the emission mechanisms at work. The following are key questions in this area which CTA will be able to address, because of the extension of the accessible energy domain, the improvement in sensitivity, and the superior angular resolution it provides:

(a) Studies of the formation of relativistic outflows from highly magnetised, rotating objects. If gamma-ray binaries are pulsars, is the gamma-ray emission coming mostly from processes within the pulsar wind zone or rather from particles accelerated in the wind collision shock? Is the answer to 
this question a function of energy? What role do the inner winds play, particularly with regard to particle injection? Gamma-ray astronomy can provide data that will help to answer these questions, but which will also throw light on the particle energy distribution within the pulsar wind zone itself. Recent Fermi-LAT results on gamma-ray binaries, such as LS I +61 303 and LS 5039 (which are found to be periodic at GeV and TeV energies, although anti-correlated [23]), show the existence of a cutoff in the SED at a few GeV (a feature that was not predicted by any models). Thus, the large energy coverage of CTA is an essential prerequisite in disentangling of the pulsed and continuous components of the radiation and the exploration of the processes leading to the observed GeV-TeV spectral differences.

(b) Studies of the link between accretion and ejection around compact objects and transient states associated with VHE emission. It is known that black holes display different spectral states in X-ray emission, with transitions between a low/hard state, where a compact radio jet is seen, to a high/soft state, where the radio emission is reduced by large factors or not detectable at all [24]. Are these spectral changes related to changes in the gamma-ray emission? Is there any gamma-ray emission during nonthermal radio flares (with increased flux by up to a factor of 1,000)? Indeed, gamma-ray emission via the inverse Compton effect is expected when flares occur in the radio to X-ray region, due to synchrotron radiation of relativistic electrons and radiative, adiabatic and energydependent escape losses in fast-expanding plasmoids (radio clouds). Can future gamma-ray observations put constraints on the magnetic fields in plasmoids?

Continued observations of key objects (such as Cyg X-1) with the sensitivity of current instruments (using sub-arrays of CTA) can provide good coverage. Flares of less than 1 hour at a flux of $10 \%$ of the Crab could be detected at the distance of the Galactic Centre. Hence variable sources could be monitored and triggers provided for observations with all CTA telescopes or with other instruments. For short flares, energy coverage in the 10-100 GeV band is not possible with current instruments (AGILE and Fermi-LAT lack sensitivity). Continuous coverage at higher energies is also impossible, due to lack of sensitivity with the current generation of Imaging Atmospheric Cherenkov Telescopes (IACTs). CTA will provide improved access to both regions.

(c) Collision of the jet with the ISM, as a non-variable source of gammaray emission. Improved angular resolution at high energies will provide opportunities for the study of microquasars, particularly if their jets contain a sizeable fraction of relativistic hadrons. While inner engines will still remain unresolved with future Cherenkov telescope arrays, microquasar jets and their interaction with the ISM might become resolvable, leading to the distinction of emission from the central object (which may be variable) and from the jet-ISM interaction (which may be stable). Microquasars, gamma-ray, and X-ray binaries, 
and high-energy aspects of astrophysical jets and binaries are discussed in [25].

\subsubsection{Stellar clusters, star formation, and starburst galaxies}

While the classical paradigm has supernova explosions as the dominant source of cosmic rays, it has been speculated that cosmic rays are also accelerated in stellar winds around massive young stars before they explode as supernovae, or around star clusters [26]. Indeed, there is growing evidence from gammaray data for a population of sources related to young stellar clusters and environments with strong stellar winds. However, lack of sensitivity currently prevents the detailed study and clear identification of these sources of gamma radiation. CTA aims at a better understanding of the relationship between star formation processes and gamma-ray emission. CTA can experimentally establish whether there is a direct correlation between star formation rate and gamma-ray luminosity when convection and absorption processes at the different environments are taken into account. Both the VERITAS and H.E.S.S. arrays have done deep observations of the nearest starburst galaxies, and have found them to be emitting $\mathrm{TeV}$ gamma-rays at the limit of their sensitivity. Future observations, with improved sensitivity at higher and lower energies, will reveal details of this radiation which in turn will help with an understanding of the spectra, provide constraints on the physical emission scenarios and extend the study of the relationship between star formation processes and gamma-ray emission to extragalactic environments. A good compendium of the current status of this topic can be found in the proceedings of a recent conference [27].

\subsubsection{Pulsar physics}

Pulsar magnetospheres are known to act as efficient cosmic accelerators, yet there is no complete and accepted model for this acceleration mechanism, a process which involves electrodynamics with very high magnetic fields as well as the effects of general relativity. Pulsed gamma-ray emission allows the separation of processes occurring in the magnetosphere from the emission in the surrounding nebula. That pulsed emission at tens of $\mathrm{GeV}$ can be detected with Cherenkov telescopes was recently demonstrated by MAGIC with the Crab pulsar [28] (and the sensitivity for pulsars with known pulse frequency is nearly an order of magnitude higher than for standard sources). Current Fermi-LAT results provide some support for models in which gamma-ray emission occurs far out in the magnetosphere, with reduced magnetic field absorption (i.e. in outer gaps). In these models, exponential cut-offs in the spectral energy distribution are expected at a few $\mathrm{GeV}$, which have already been found in several Fermi pulsars. To make further progress in understanding the emission mechanisms in pulsars it is necessary to study their radiation at extreme energies. In particular, the characteristics of pulsar emission in 
the $\mathrm{GeV}$ domain (currently best examined by the Fermi-LAT) and at VHE will tell us more about the electrodynamics within their magnetospheres. Studies of interactions of magnetospheric particle winds with external ambient fields (magnetic, starlight, CMB) are equally vital. Between $\sim 10 \mathrm{GeV}$ and $\sim 50 \mathrm{GeV}$ (where the LAT performance is limited) CTA, with a special lowenergy trigger for pulsed sources, will allow a closer look at unidentified Fermi sources and deeper analysis of Fermi pulsar candidates. Above $50 \mathrm{GeV}$ CTA will explore the most extreme energetic processes in millisecond pulsars. The VHE domain will be particularly important for the study of millisecond pulsars, very much as the HE domain (with Fermi) is for classical pulsars. On the other hand, the high-energy emission mechanism from magnetars is essentially unknown. For magnetars, we do not expect polar cap emission. Due to the large magnetic field, all high-energy photons would be absorbed if emitted close to the neutron star, i.e., CTA would be testing outer-gap models, especially if large $\mathrm{X}$-ray flares are accompanied by gamma-emission.

CTA can study the GeV-TeV emission related to short-timescale pulsar phenomena, which is beyond the reach of currently working instruments. CTA can observe possible high-energy phenomena related to timing noise (in which the pulse phase and/or frequency of radio pulses drift stochastically) or to sudden increases in the pulse frequency (glitches) produced by apparent changes in the momentum of inertia of neutron stars.

Periodicity measurements with satellite instruments, which require very long integration times, may be compromised by such glitches, while CTA, with its much larger detection area and correspondingly shorter measurement times, is not.

A good compendium of the current status of this topic can be found in the proceedings and the talks presented at the "International Workshop on the High-Energy Emission from Pulsars and their Systems" [29].

\subsubsection{Active galaxies, cosmic radiation fields and cosmology}

Active Galactic Nuclei (AGN) are among the largest storehouses of energy known in our cosmos. At the intersection of powerful low-density plasma inflows and outflows, they offer excellent conditions for efficient particle acceleration in shocks and turbulences. AGN represent one third of the known VHE gamma-ray sources, with most of the detected objects belonging to the BL Lac class. The fast variability of the gamma-ray flux (down to minute time scales) indicates that gamma-ray production must occur close to the black hole, assisted by highly relativistic motion resulting in time (Lorentz) contraction when viewed by an observer on Earth. Details of how these jets are launched or even the types of particles of which they consist are poorly known. Multi-wavelength observations with high temporal and spectral resolution can help to distinguish between different scenarios, but this is at the limit of the capabilities of current instruments. The sensitivity of CTA, combined with simultaneous observations in other wavelengths, will provide a crucial advance in understanding the mechanisms driving these sources. 
Available surveys of BL Lacs suffer several biases at all wavelengths, further complicated by Doppler boosting effects and high variability. The big increase of sensitivity of CTA will provide large numbers of VHE sources of different types and opens the way to statistical studies of the VHE blazar and AGN populations. This will enable the exploration of the relation between different types of blazars, and of the validity of unifying AGN schemes. The distribution in redshift of known and relatively nearby BL Lac objects peaks around $z \sim 0.3$. The large majority of the population is found within $z<1$, a range easily accessible with CTA. CTA will therefore be able to analyse in detail blazar populations (out to $z \sim 2$ ) and the evolution of AGN with redshift and to start a genuine "blazar cosmology".

Several scenarios have been proposed to explain the VHE emission of blazars. ${ }^{3}$ However, none of them is fully self-consistent, and the current data are not sufficient to firmly rule out or confirm a particular mechanism. In the absence of a convincing global picture, a first goal for CTA will be to constrain model-dependent parameters of blazars within a given scenario. This is achievable due to the wide energy range, high sensitivity and high spectral resolution of CTA combined with multi-wavelength campaigns. Thus, the physics of basic radiation models will be constrained by CTA, and some of the models will be ruled out. A second more difficult goal will be to distinguish between the different remaining options and to firmly identify the dominant radiation mechanisms. Detection of specific spectral features, breaks, cut-offs, absorption or additional components, would be greatly helpful for this. The role of CTA as a timing explorer will be decisive for constraining both the radiative phenomena associated with, and the global geometry and dynamics of, the AGN engine. Probing variability down to the shortest time scales will significantly constrain acceleration and cooling times, instability growth rates, and the time evolution of shocks and turbulences. For the brightest blazar flares, current instruments are able to detect variability on the scales of several minutes. With CTA, such flares should be detectable within seconds, rather than minutes. A study of the minimum variability times of AGN with CTA would allow the localisation of VHE emission regions (parsec distance scales in the jet, the base of the jet, or the central engine) and would provide stringent constraints on the emission mechanisms as well as the intrinsic time scale connected to the size of the central super-massive black hole.

Recently, radio galaxies have emerged as a new class of VHE emitting AGN [37]. Given the proximity of the sources and the larger jet angle to the line of sight compared to BL Lac objects, the outer and inner kpc jet structures will be

\footnotetext{
${ }^{3}$ There are several clear cases of blazar SEDs where the X-ray peak and the $\gamma$-ray peak, with their correlated luminosity and spectral changes, are interpreted within a synchrotron-self-Compton (SSC) model [30] as the synchrotron and IC peak respectively, produced by a time-varying population of particles (e.g. [31, 32]). A variant of the Compton scenario considers that the soft photons produced externally to the jet may be more effective than the internal ones (external Compton, EC, model; e.g. [33, 34]). Models based on hadronic acceleration (e.g. [35, 36]) can also reproduce the blazar SEDs and lightcurves.
} 
spatially resolved by CTA. This will allow precise location of the main emission site and searches for VHE radiation from large-scale jets and hot spots besides the central core and jets seen in very long baseline interferometry images.

The observation of VHE emission from distant objects and their surroundings will also offer the unique opportunity to study extragalactic magnetic fields at large distances. If the fields are large, an $e^{+} e^{-}$pair halo forms around AGNs, which CTA, with its high sensitivity and extended field of view, should be capable of detecting. For smaller magnetic field values, the effect of $e^{+} e^{-}$ pair formation along the path to the Earth is seen through energy-dependent time-delays of variable VHE emission, which CTA with its excellent time resolution will be ideally suited to measure.

CTA will also have the potential to deliver for the first time significant results on extragalactic diffuse emission at VHE, and offers the possibility of probing the integrated emission from all sources at these energies. While well measured at $\mathrm{GeV}$ energies with the EGRET and Fermi-LAT instruments, the diffuse emission at VHE is extremely challenging to measure due to its faintness and the difficulty of adequately subtracting the background. Here, the improved sensitivity coupled with the large field of view puts detection in reach of CTA.

VHE gamma-rays traveling from remote sources interact with the EBL via $e^{+} e^{-}$pair production and are absorbed. Studying such effects as a function of the energy and redshift will provide unique information on the EBL density, and thereby on the history of the formation of stars and galaxies in the Universe. This approach is complementary to direct EBL measurements, which are hampered by strong foreground emission from our planetary system (zodiacal light) and the Galaxy.

We anticipate that MAGIC II and H.E.S.S. II will at least double the number of detected sources, but this is unlikely to resolve the ambiguity between intrinsic spectral features and effects due to the EBL. It would still be very difficult to extract spectral information beyond $z>0.5$, if our current knowledge of the EBL is correct. Only CTA will be able to provide a sufficiently large sample of VHE gamma-ray sources, and high-quality spectra for individual objects. For many of the sources, the SED will be determined at $\mathrm{GeV}$ energies, which are much less affected by the absorption and, thus, more suitable for the study of the intrinsic properties of the objects. We therefore anticipate that with CTA it will be possible to make robust predictions about the intrinsic spectrum above $40-50 \mathrm{GeV}$, for individual sources and for particular source classes.

The end of the dark ages of the Universe, the epoch of reionisation, is a topic of great interest [38]. Not (yet) fully accessible via direct observations, most of our knowledge comes from simulations and from integral observables like the cosmic microwave background. The first (Population III) and second generations of stars are natural candidates for being the source of reionisation. If the first stars are hot and massive, as predicted by simulations, their UV photons emitted at $z>5$ would be redshifted to the near infrared and could leave a unique signature on the EBL spectrum. If the EBL contribution from 
lower redshift galaxies is sufficiently well known (for example, as derived from source counts) upper limits on the EBL density can be used to probe the properties of early stars and galaxies. Combining detailed model calculations with redshift-dependent EBL density measurements could allow the probing of the reionisation/ionisation history of the Universe. A completely new wavelength region of the EBL will be opened up by observations of sources at very high redshifts $(z>5)$, which will most likely be gamma-ray bursts. According to high-redshift UV background models, consistent with our current knowledge of cosmic reionisation, spectral cut-offs are expected in the few $\mathrm{GeV}$ to few tens of $\mathrm{GeV}$ range at $z>5$. Thus, CTA could have the unique potential to probe cosmic reionisation models through gamma-ray absorption in high-z GRBs. We analyse the GRB prospects in more detail in the following.

A good compendium of the current state of this topic can be found in the talks and the proceedings of the meeting, High-energy phenomena in relativistic outflows II [39].

\subsubsection{Gamma-ray bursts}

Gamma-Ray Bursts are the most powerful explosions in the Universe, and are by far the most electromagnetically luminous sources known to us. The peak luminosity of GRBs, equivalent to the light from millions of galaxies, means they can be detected up to high redshifts, hence act as probes of the star formation history and reionisation of the Universe. The highest measured GRB redshift is $z=8.2$ but GRBs have been observed down to $z=0.0085$ (the mean redshift is $z \sim 2.2$ ). GRBs occur in random directions on the sky, briefly outshining the rest of the hard X-ray and soft gamma-ray sky, and then fade from view. The rapid variability seen in gamma- and X-rays indicates a small source size, which together with their huge luminosities and clearly non-thermal spectrum (with a significant high-energy tail) require the emitting region to move toward us with a very large bulk Lorentz factor of typically $>100$, sometimes as high as $>1,000$ [40-42].

Thus, GRBs are thought to be powered by ultra-relativistic jets produced by rapid accretion onto a newly formed stellar-mass black hole or a rapidly rotating highly-magnetised neutron star (i.e. a millisecond magnetar). The prompt gamma-ray emission is thought to originate from dissipation within the original outflow by internal shocks or magnetic reconnection events. Some long duration GRBs are clearly associated with core-collapse supernovae of type Ic (from very massive Wolf-Rayet stars stripped of their $\mathrm{H}$ and $\mathrm{He}$ envelope by strong stellar winds), while the progenitors of short GRBs are much less certain: the leading model involves the merger of two neutron stars or a neutron star and a black hole [43, 44].

Many of the details of GRB explosions remain unclear. Studying them requires a combination of rapid observations to observe the prompt emission before it fades, and a wide energy range to properly capture the spectral energy distribution. Most recently, GRBs have been observed by the Swift and Fermi missions, which have revealed an even more complex behaviour 
than previously thought, featuring significant spectral and temporal evolution. As yet, no GRB has been detected at energies $>100 \mathrm{GeV}$ due to the limited sensitivity of current instruments and the large typical redshifts of these events. In just over a year of operation, the Fermi-LAT has detected emission above $10 \mathrm{GeV}$ (30 GeV) from 4 (2) GRBs. In many cases, the LAT detects emission $>0.1 \mathrm{GeV}$ for several hundred seconds in the GRB rest-frame. In GRB090902B a photon of energy $\sim 33.4 \mathrm{GeV}$ was detected, which translates to an energy of $\sim 94 \mathrm{GeV}$ at its redshift of $z=1.822$. Moreover, the observed spectrum is fairly hard up to the highest observed energies.

Extrapolating the Fermi spectra to CTA energies suggests that a good fraction of the bright LAT GRBs could be detected by CTA even in $\sim$ minute observing times, if it could be turned to look at the prompt emission fast enough. The faster CTA could get on target, the better the scientific return. Increasing the observation duty cycle by observing for a larger fraction of the lunar cycle and at larger zenith angles could also increase the return.

Detecting GRBs in the CTA energy range would greatly enhance our knowledge of the intrinsic spectrum and the particle acceleration mechanism of GRBs, particularly when combined with data from Fermi and other observatories. As yet it is unclear what the relative importance is of the various proposed emission processes, which divide mainly into leptonic (synchrotron and inverse-Compton, and in particular synchrotron-self-Compton) and hadronic processes (induced by protons or nuclei at very high energies which either radiate synchrotron emission or produce pions with subsequent electromagnetic cascades). CTA may help to determine the identity of the distinct high-energy component that was observed so far in three out of the four brightest LAT GRBs. The origin of the high-energy component may in turn shed light on the more familiar lower-energy components that dominate at soft gammaray energies. The bulk Lorentz factor and the composition (protons, $e^{+} e^{-}$ pairs, magnetic fields) of the outflows are also highly uncertain and may be probed by CTA. The afterglow emission which follows the prompt emission is significantly fainter, but should also be detectable in some cases. Such detections would be expected from bright GRBs at moderate redshift, not only from the afterglow synchrotron-self-Compton component, but perhaps also from inverse-Compton emission triggered by bright, late (hundreds to thousands of seconds) flares that are observed in about half of all Swift GRBs.

The discovery space at high energies is large and readily accessible to CTA. The combination of GRBs being extreme astrophysical sources and cosmological probes make them prime targets for all high-energy experiments. With its large collecting area, energy range and rapid response, CTA is by far the most powerful and suitable VHE facility for GRB research and will open up a new energy range for their study.

\subsubsection{Galaxy clusters}

Galaxy clusters are storehouses of cosmic rays, since all cosmic rays produced in the galaxies of the cluster since the beginning of the Universe will be 
confined there. Probing the density of cosmic rays in clusters via their gammaray emission thus provides a calorimetric measure of the total integrated nonthermal energy output of galaxies. Accretion/merger shocks outside cluster galaxies provide an additional source of high-energy particles. Emission from galaxy clusters is predicted at levels just below the sensitivity of current instruments [45].

Clusters of galaxies are the largest, gravitationally-bound objects in the Universe. The observation of mainly radio (and in some cases X-ray) emission proves the existence of non-thermal phenomena therein, but gamma-rays have not yet been detected. A possible additional source of non-thermal radiation from clusters is the annihilation of dark matter (DM). The increased sensitivity of CTA will help to establish the DM signal, and CTA could possibly be the first instrument to map DM at the scale of galaxy clusters.

\subsubsection{Dark matter and fundamental physics}

The dominant form of matter in the Universe is the as yet unknown dark matter, which is most likely to exist in the form of a new class of particles such as those predicted in supersymmetric or extra dimensional extensions to the standard model of particle physics. Depending on the model, these DM particles can annihilate or decay to produce detectable Standard Model particles, in particular gamma-rays. Large dark matter densities due to the accumulation in gravitational potential wells leads to detectable fluxes, especially for annihilation, where the rate is proportional to the square of the density. CTA is a discovery instrument with unprecedented sensitivity for this radiation and also an ideal tool to study the properties of the dark matter particles. If particles beyond the standard model are discovered (at the Large Hadron Collider or in underground experiments), CTA will be able to verify whether they actually form the dark matter in the Universe. Slow-moving dark matter particles could give rise to a striking, almost mono-energetic photon emission. The discovery of such line emission would be conclusive evidence for dark matter. CTA might have the capability to detect gamma-ray lines even if the cross-section is loop-suppressed, which is the case for the most popular candidates of dark matter, i.e. those inspired by the minimal supersymmetric extensions to the standard model (MSSM) and models with extra dimensions, such as KaluzaKlein theories. Line radiation from these candidates is not detectable by Fermi, H.E.S.S. II or MAGIC II, unless optimistic assumptions on the dark matter density distribution are made. Recent updates of calculations regarding the gamma-ray spectrum from the annihilation of MSSM dark matter indicate the possibility of final-state contributions giving rise to distinctive spectral features (see the reviews in [46]).

The more generic continuum contribution (arising from pion production) is more ambiguous but, with its curved shape, potentially distinguishable from the usual power-law spectra exhibited by known astrophysical sources.

Our galactic centre is one of the most promising regions to look for dark matter annihilation radiation due to its predicted very high dark matter 
density. It has been observed by many experiments so far (e.g. H.E.S.S., MAGIC and VERITAS) and high-energy gamma emission has been found. However, the identification of dark matter in the galactic centre is complicated by the presence of many conventional source candidates and the difficulties of modelling the diffuse gamma-ray background adequately. The angular and energy resolution of CTA, as well as its enhanced sensitivity will be crucial to disentangling the different contributions to the radiation from the galactic centre.

Other individual targets for dark matter searches are dwarf spheroidals and dwarf galaxies. They exhibit large mass-to-light ratios, and allow dark matter searches with low astrophysical backgrounds. With H.E.S.S., MAGIC and Fermi-LAT, some of these objects were observed and upper limits on dark matter annihilation calculated, which are currently about an order of magnitude above the prediction of the most relevant cosmological models. CTA will have good sensitivity for Weakly Interacting Massive Particle (WIMP) annihilation searches in the low and medium energy domains. An improvement in flux sensitivity of 1-2 orders of magnitude over current instruments is expected. Thus CTA will allow tests in significant regions of the MSSM parameter space.

Dark matter would also cause spectral and spatial signatures in extragalactic and galactic diffuse emission. While the emissivity of conventional astrophysical sources scale with the local matter density, the emissivity of annihilating dark matter scales with the density squared, causing differences in the small-scale anisotropy power spectrum of the diffuse emission.

Recent measurements of the positron fraction presented by the PAMELA Collaboration [47] point towards a relatively local source of positrons and electrons, especially if combined with the measurement of the $e^{+} e^{-}$spectrum by Fermi-LAT [48]. The main candidates being put forward are either pulsar(s) or dark matter annihilation. One way to distinguish between these two hypotheses is the spectral shape. The dark matter spectrum exhibits a sudden drop at an energy which corresponds to the dark matter particle mass, while the pulsar spectrum falls off more smoothly. Another hint is a small anisotropy, either in the direction of the galactic centre (for dark matter) or in the direction of the nearest mature pulsars. The large effective area of CTA, about six orders of magnitudes larger than for balloon- and satellite-borne experiments, and the greatly improved performance compared to existing Cherenkov observatories, might allow the measurement of the spectral shape and even the tiny dipole anisotropy.

If the PAMELA result originated from dark matter, the DM particle's mass would be $>1 \mathrm{TeV} / \mathrm{c}^{2}$, i.e. large in comparison to most dark matter candidates in MSSM and Kaluza-Klein theories. With its best sensitivity at $1 \mathrm{TeV}$, CTA would be well suited to detect dark matter particles of $\mathrm{TeV} / \mathrm{c}^{2}$ masses. The best sensitivity of Fermi-LAT for dark matter is at masses of the order of 10-100 $\mathrm{GeV} / \mathrm{c}^{2}$.

Electrons and positrons originating from dark matter annihilation or decay also produce synchrotron radiation in the magnetic fields present in the dense 
regions where the annihilation might take place. This opens up the possibility of multi-wavelength observations. Regardless of the wavelength domain in which dark matter will be detectable using present or future experiments, it is evident that CTA will provide coverage for the highest-energy part of the multi-wavelength spectrum necessary to pinpoint, discriminate and study dark matter indirectly.

Due to their extremely short wavelength and long propagation distances, very high-energy gamma-rays are sensitive to the microscopic structure of space-time. Small-scale perturbations of the smooth space-time continuum should manifest themselves in an (extremely small) energy dependence of the speed of light. Such a violation of Lorentz invariance, on which the theory of special relativity is based, is present in some quantum gravity (QG) models. Burst-like events in which gamma-rays are produced, e.g. in active galaxies, allow this energy-dependent dispersion of gamma-rays to be probed and can be used to place limits on certain classes of quantum gravity scenarios, and may possibly lead to the discovery of effects associated with Planck-scale physics.

CTA has the sensitivity to detect characteristic time-scales and QG effects in AGN light curves (if indeed any exist) on a routine basis without exceptional source flux states and in small observing windows. CTA can resolve time scales as small as few seconds in AGN light curves and QG effects down to $10 \mathrm{~s}$. Very good sensitivity at energies $>1 \mathrm{TeV}$ is especially important to probe the properties of QG effects at higher orders. Fermi recently presented results based on observations of a GRB which basically rule out linear-inenergy variations of the speed of light up to $1.2 \times$ the Planck scale [49] To test quadratic or higher order dependencies the sensitivity provided by CTA will be needed.

This topic is thoroughly discussed in the book "Particle dark matter" edited by G. Bertone [46], and aspects of the fundamental physics implications of VHE gamma-ray observations are covered in a recent review [50].

\subsubsection{Imaging stars and stellar surfaces}

The quest for better angular resolution in astronomy is driving much of the instrumentation developments throughout the world, from gamma-rays through low-frequency radio waves. The optical region is optimal for studying objects with stellar temperatures, and the current frontier in angular resolution is represented by optical interferometers such as ESO's VLTI in Chile or the CHARA array in California. Recently, these have produced images of giant stars surrounded by ejected gas shells and revealed the oblate shapes of stars deformed by rapid rotation. However, such phase interferometers are limited by atmospheric turbulence to baselines of no more than some $100 \mathrm{~m}$, and to wavelengths longer than the near infrared. Only very few stars are large enough to be imaged by current facilities. To see smaller details (e.g. magnetically active regions, planet-forming disks obscuring parts of the stellar disk) requires interferometric baselines of the order of $1 \mathrm{~km}$. It has been proposed to incorporate such instruments on ambitious future space missions 
(Luciola Hypertelescope for the ESA Cosmic Vision; Stellar Imager as a NASA vision mission), or to locate them on the Earth in regions with the bestpossible seeing, e.g. in Antarctica (KEOPS array). However, the complexity and cost of these concepts seems to put their realisation beyond the immediate planning horizon.

An alternative that can be realised much sooner is offered by CTA, which could become the first kilometre-scale optical imager. With many telescopes distributed over a square $\mathrm{km}$ or more, its unprecedented optical collecting area forms an excellent facility for ultrahigh angular resolution (sub-milliarcsecond) optical imaging through long-baseline intensity interferometry. This method was originally developed by Hanbury Brown and Twiss in the 1950s [51] for measuring the sizes of stars. It has since been extensively used in particle physics ("HBT interferometry") but it has had no recent application in astronomy because it requires large telescopes spread out over large distances, which were not available until the recent development of atmospheric Cherenkov telescopes.

The great observational advantages of intensity interferometry are its lack of sensitivity to atmospheric disturbances and to imperfections in the optical quality of the telescopes. This is because of the electronic (rather than optical) connection of telescopes. The noise relates to electronic timescales of nanoseconds (and light-travel distances of centimetres or metres) rather than to those of the light wave itself (femtoseconds and nanometres).

The requirements are remarkably similar to those for studying Cherenkov light: large light-collecting telescopes, high-speed optical detectors with sensitivity extending into the blue, and real-time handling of the signals on nanosecond levels. The main difference to ordinary Cherenkov Telescope operation lies in the subsequent signal analysis which digitally synthesises an optical telescope. From the viewpoint of observatory operations, it is worth noting that bright stars can be measured for interferometry during bright-sky periods of full Moon, which would hamper Cherenkov studies.

Science targets include studying the disks and surfaces of hot and bright stars [52, 53] Rapidly rotating stars naturally take on an oblate shape, with an equatorial bulge that, for stars rotating close to their break-up speed, may extend into a circumstellar disk, while the regions with higher effective gravity near the stellar poles become overheated, driving a stellar wind. If the star is observed from near its equatorial plane, an oblate image results. If the star is instead observed from near its poles, a radial temperature gradient should be seen. Possibly, stars with rapid and strong differential rotation could take on shapes, midway between that of a doughnut and a sphere. The method permits studies in both broad-band optical light and in individual emission lines, and enables the mapping of gas flows between the components in close binary stars.

\subsubsection{Measurements of charged cosmic rays}

Cherenkov telescopes can contribute to cosmic ray physics by detecting these particles directly [54]. CTA can provide measurements of the spectra of 
cosmic-ray electrons and nuclei in the energy regime where balloon- and spaceborne instruments run out of data. The composition of cosmic rays has been measured by balloon- and space-borne instruments (e.g. TRACER) up to $\approx 100 \mathrm{TeV}$. Starting at about $1 \mathrm{PeV}$ instruments can detect air showers at ground level (e.g. KASCADE). Such air shower experiments have, however, difficulties in identifying individual nuclei, and consequently their composition results are of lower resolution than direct measurements. Cherenkov telescopes are the most promising candidates to close the experimental gap between the $\mathrm{TeV}$ and $\mathrm{PeV}$ domains, and will probably achieve better mass resolution than ground based particle arrays. Additionally, CTA can perform crucial measurements of the spectrum of cosmic-ray electrons. TeV electrons have very short lifetimes and thus propagation distances due to their rapid energy loss. The upper end of the electron spectrum (which is not accessible by current balloon and satellite experiments) is therefore expected to be dominated by local electron accelerators and the cosmic-ray electron spectrum can provide valuable information about characteristics of the contributing sources and of the electron propagation. While such measurements involve analyses that differ from the conventional gamma-ray studies, a proof-ofprinciple has already been performed with the H.E.S.S. telescopes. Spectra of electrons and iron nuclei have been published [55]. The increase in sensitivity expected from CTA will provide significant improvements in such measurements.

\subsection{The CTA legacy}

The CTA legacy will most probably not be limited to individual observations addressing the issues mentioned above, but also comprise a survey of the inner Galactic plane and/or, depending on the final array capabilities, a deep survey of all or part of the extragalactic sky. Surveys provide coverage of large parts of the sky, maximise serendipitous detections, allow for optimal use of telescope time, and thereby ensure the legacy of the project for the future scientific community. Surveys of different extents and depths are among the scientific goals of all major facilities planned or in operation at all wavelengths. In view of both H.E.S.S. (see Fig. 2) and Fermi-LAT survey results, the usefulness of surveys is unquestioned, and many of the scientific cases discussed above can be encompassed within such an observational strategy.

Two possible CTA survey schemes have been studied to date:

- All-sky survey: With an effective field-of-view of $5^{\circ}, 500$ pointings of 0.5 hours would cover a survey area of a quarter of the sky at the target sensitivity of $0.01 \mathrm{Crab}$. Hence, using about a quarter of the observing time in a year, a quarter of the sky can be surveyed down to a level of $<0.01$ $\mathrm{Crab}$, which is equivalent to the flux level of the faintest AGN currently detected at VHE energies.

- Galactic plane survey: The H.E.S.S. Galactic plane survey covered $1.5 \%$ of the sky, at a sensitivity of 0.02 Crab above $200 \mathrm{GeV}$, using about 250 hours 
of observing time. The increase in CTA sensitivity means that a similar investment in time can be expected to result in a sensitivity of 2-3 mCrab over the accessible region of the Galactic plane.

The high-energy phenomena which can be studied with CTA span a wide field of galactic and extragalactic astrophysics, of plasma physics, particle physics, dark matter studies, and investigations of the fundamental physics of spacetime. They carry information on the birth and death of stars, on the matter circulation in the Galaxy, and on the history of the Universe. Optimisation of the layout of CTA with regards to these different science goals is a difficult task and detailed studies of the response of different array configurations to these scientific problems being conducted during the Design Study and the Preparatory Phase.

\section{Advancing VHE gamma-ray astronomy with CTA}

The latest generation of ground-based gamma-ray instruments (H.E.S.S., MAGIC, VERITAS, Cangaroo III (http://icrhp9.icrr.u-tokyo.ac.jp) and MILAGRO (http://www.lanl.gov/milagro)) allow the imaging, photometry and spectroscopy of sources of high energy radiation and have ensured that VHE gamma ray studies have grown to become a genuine branch of astronomy. The number of known sources of VHE gamma rays is exceeding 100, and source types include supernovae, pulsar wind nebulae, binary systems, stellar winds, various types of active galaxies and unidentified sources without obvious counterparts. H.E.S.S. has conducted a highly successful survey of the Milky Way covering about 600 square degrees, which resulted in the detection of tens of new sources. However, a survey of the full visible sky would require at least a decade of observations, which is not feasible.

Due to the small fluxes, instruments for detection of high-energy gamma rays (above some $10 \mathrm{GeV}$ ) require a large effective detection area, eliminating space-based instruments which directly detect the incident gamma rays. Ground-based instruments allow much larger detection areas. They measure the particle cascade induced when a gamma ray is absorbed in the atmosphere, either by using arrays of particle detectors to record the cascade particles which reach the ground (or mountain altitudes), or by using Cherenkov telescopes to image the Cherenkov light emitted by secondary electrons and positrons in the cascade.

Compared to Cherenkov telescopes, air shower arrays (such as MILAGRO, AS-gamma or ARGO) have the advantage of a large duty cycle-they can observe during the daytime - and of a large solid angle coverage. However, their current sensitivity is such that they can only detect sources with a flux around the level of the flux from the Crab Nebula, the strongest known steady source of VHE gamma rays. Results from air shower arrays demonstrate that there are relatively few sources emitting at this level. The recent rapid evolution of VHE gamma-ray astronomy was therefore primarily driven by 
Table 1 Properties of selected air-Cherenkov instruments, including two of historical interest (HEGRA and CAT)

\begin{tabular}{|c|c|c|c|c|c|c|c|c|c|c|}
\hline \multirow[t]{2}{*}{ Instrument } & \multirow{2}{*}{$\begin{array}{l}\text { Lat } \\
\left(^{\circ}\right)\end{array}$} & \multirow{2}{*}{$\begin{array}{l}\text { Long } \\
\left(^{\circ}\right)\end{array}$} & \multirow{2}{*}{$\begin{array}{l}\text { Alt } \\
(\mathrm{m})\end{array}$} & \multicolumn{3}{|c|}{ Telescopes } & \multirow[t]{2}{*}{ Pixels } & \multirow{2}{*}{$\begin{array}{l}\text { FoV } \\
\left(^{\circ}\right)\end{array}$} & \multirow{2}{*}{$\begin{array}{l}\text { Thresh } \\
(\mathrm{TeV})\end{array}$} & \multirow{2}{*}{$\begin{array}{l}\text { Sensitivity } \\
\text { (\% Crab) }\end{array}$} \\
\hline & & & & \# & $\begin{array}{l}\text { Area } \\
\left(\mathrm{m}^{2}\right)\end{array}$ & $\begin{array}{l}\text { Total } \\
\left(\mathrm{m}^{2}\right)\end{array}$ & & & & \\
\hline H.E.S.S. & -23 & 16 & 1,800 & 4 & 107 & 428 & 960 & 5 & 0.1 & 0.7 \\
\hline VERITAS & 32 & -111 & 1,275 & 4 & 106 & 424 & 499 & 3.5 & 0.1 & 0.7 \\
\hline MAGIC I $^{\mathrm{a}}+\mathrm{II}$ & 29 & 18 & 2,225 & 2 & 234 & 468 & $576 / 1039$ & 3.5 & 0.03 & 1.0 \\
\hline CANGAROO-III & -31 & 137 & 160 & 3 & 57.3 & 172 & 427 & 4 & 0.4 & 15 \\
\hline Whipple ${ }^{\mathrm{a}}$ & 32 & -111 & 2,300 & 1 & 75 & 75 & 379 & 2.3 & 0.3 & 15 \\
\hline HEGRA & 29 & 18 & 2,200 & 5 & 8.5 & 43 & 271 & 4.3 & 0.5 & 5 \\
\hline CAT $^{\mathrm{a}}$ & 42 & 2 & 1,650 & 1 & 17.8 & 17.8 & 600 & 4.8 & 0.25 & 15 \\
\hline
\end{tabular}

Adapted from [56]

${ }^{a}$ These instruments have pixels of two different sizes

Cherenkov instruments, which reach sensitivities of $1 \%$ of the Crab flux for typical observing times of $25 \mathrm{~h}$, and which provide significantly better angular resolution. While there are proposals for better air shower arrays with improved sensitivity (e.g. the HAWC project), which will certainly offer valuable complementary information, such approaches will not be able to compete in sensitivity with next-generation Cherenkov telescopes.

The properties of the major current and historic Cherenkov instruments are listed in Table 1. The instruments consist of up to four Cherenkov telescopes (or 5 for the H.E.S.S. II upgrade). They reach sensitivities of about $1 \%$ of the flux of the Crab Nebula at energies in the $100 \mathrm{GeV}-1 \mathrm{TeV}$ range. Sensitivity degrades towards lower energies, due to threshold effects, and towards higher energies, due to the limited detection area. A typical angular resolution is $0.1^{\circ}$ or slightly better for single gamma rays. Sufficiently intense sources can be located with a precision of $10-20^{\prime \prime}$.

All these instruments are operated by the groups who built them, with very limited access for external observers and no provision for open data access. Such a mode is appropriate for current instruments, which detect a relatively limited number of sources, and where the analysis and interpretation can be handled by the manpower and experience accumulated in these consortia. However, a different approach is called for in next-generation instruments, with their expected ten-fold increase in the number of detectable objects. CTA will advance the state of the art in astronomy at the highest energies of the electromagnetic spectrum in a number of decisive areas, all of which are unprecedented in this field:

European and international integration CTA will for the first time bring together and combine the experience of all virtually all groups world-wide working with atmospheric Cherenkov telescopes.

Performance of the instrument CTA aims to provide full-sky view, from a southern and a northern site, with unprecedented sensitivity, spectral coverage, angular and timing resolution, combined with a high degree of flexibility of operation. Details are addressed below. 
Operation as an open observatory The characteristics listed above imply that CTA will, for the first time in this field, be operated as a true observatory, open to the entire astrophysics (and particle physics) community, and providing support for easy access and analysis of data. Data will be made publicly available and will be accessible through Virtual Observatory tools. Service to professional astronomers will be supplemented by outreach activities and interfaces for laypersons to the data.

Technical implementation, operation, and data access While based on existing and proven techniques, the goals of CTA imply significant advances in terms of efficiency of construction and installation, in terms of the reliability of the telescopes, and in terms of data preparation and dissemination. With these characteristics, the CTA observatory is qualitatively different from experiments such as H.E.S.S., MAGIC or VERITAS and the increase in capability goes well beyond anything that could ever be achieved through an expansion or upgrade of existing instruments.

Science performance goals for CTA include in particular:

Sensitivity CTA will be about a factor of 10 more sensitive than any existing instrument. It will therefore for the first time allow detection and in-depth study of large samples of known source types, will explore a wide range of classes of suspected gamma-ray emitters beyond the sensitivity of current instruments, and will be sensitive to new phenomena. In its core energy range, from about $100 \mathrm{GeV}$ to several $\mathrm{TeV}$, CTA will have milli-Crab sensitivity, a factor of 1,000 below the strength of the strongest steady sources of VHE gamma rays, and a factor of 10,000 below the highest fluxes measured in bursts. This dynamic range will not only allow study of weaker sources and of new source types, it will also reduce the selection bias in the taxonomy of known types of sources.

Energy range Wide-band coverage of the electromagnetic spectrum is crucial for understanding the physical processes in sources of high-energy radiation. CTA is aiming to cover, with a single facility, three to four orders of magnitude in energy range. Together with the much improved precision and lower statistical errors, this will enable astrophysicists to distinguish between key hypotheses such as the leptonic or hadronic origin of gamma rays from supernovae. Combined with the Fermi gamma-ray observatory in orbit, an unprecedented seamless coverage of more than seven orders of magnitude in energy can be achieved.

Angular resolution Current instruments are able to resolve extended sources, but they cannot probe the fine structures visible in other wavebands. In supernova remnants, for example, the exact width of the gamma-ray emitting shell would provide a sensitive probe of the acceleration mechanism. Selecting a subset of gamma-ray induced cascades detected simultaneously by many of its telescopes, CTA can reach angular resolutions in the arcminute range, a factor of 5 better than the typical values for current instruments. 
Temporal resolution With its large detection area, CTA will resolve flaring and time-variable emission on sub-minute time scales, which are currently not accessible. In gamma-ray emission from active galaxies, variability time scales probe the size of the emitting region. Current instruments have already detected flares varying on time scales of a few minutes, requiring a paradigm shift concerning the phenomena in the vicinity of the supermassive black holes at the cores of active galaxies, and concerning the jets emerging from them. CTA will also enable access to episodic and periodic phenomena such as emission from inner stable orbits around black holes or from pulsars and other objects where frequent variations and glitches in period smear the periodicity when averaging over longer periods.

Flexibility Consisting of a large number of individual telescopes, CTA can be operated in a wide range of configurations, allowing on the one hand the in-depth study of individual objects with unprecedented sensitivity, and on the other hand the simultaneous monitoring of tens of potentially flaring objects, and any combination in between (see Fig. 3).

Survey capability A consequence of this flexibility is the dramatically enhanced survey capability of CTA. Groups of telescopes can point at adjacent fields in the sky, with their fields of view overlapping, providing an increase of
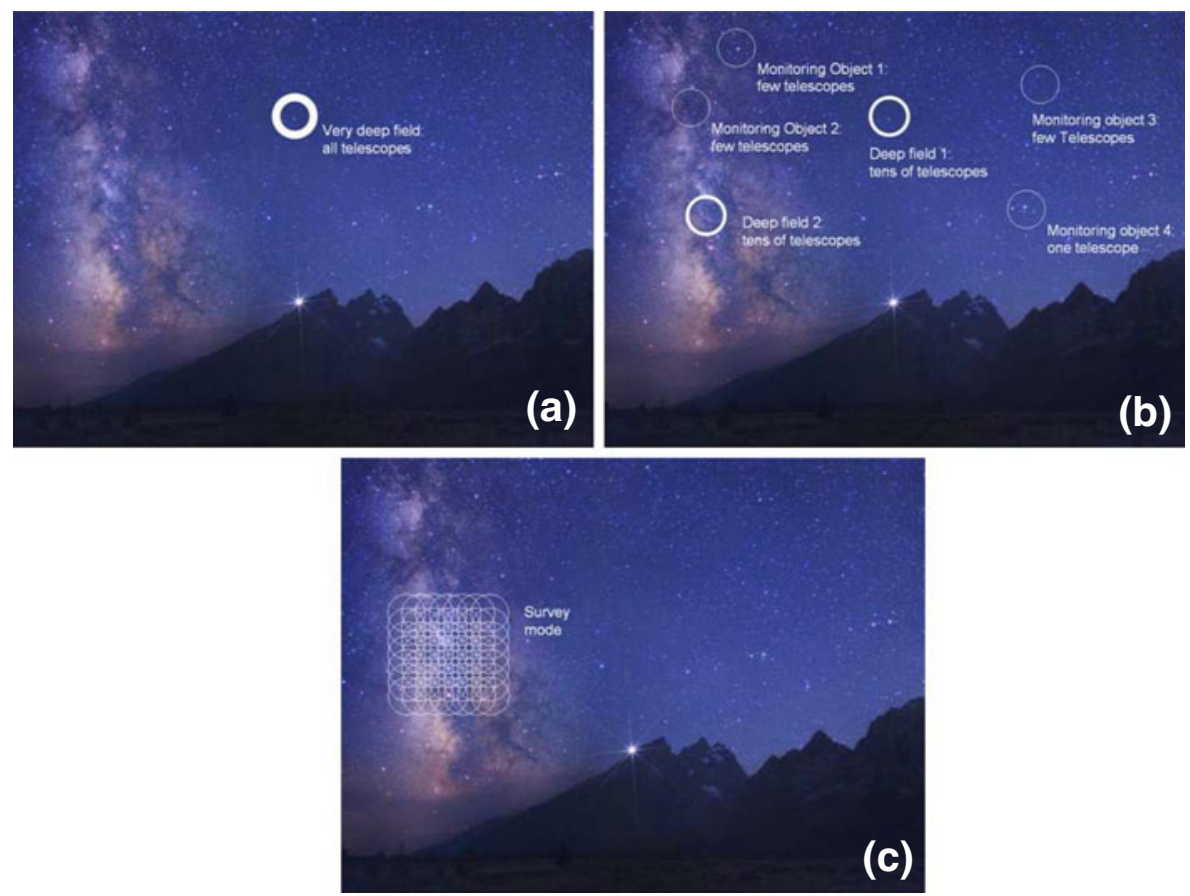

Fig. 3 Some of the possible operating modes of CTA: a very deep observations, b combining monitoring of flaring sources with deep observations, $\mathbf{c}$ a survey mode allowing full-sky surveys 
sky area surveyed per unit time by an order of magnitude, and for the first time enabling a full-sky survey at high sensitivity.

Number of sources Extrapolating from the intensity distribution of known sources, CTA is expected to enlarge the catalogue of objects detected from currently several tens of objects to about 1,000 objects.

Global coverage and integration Ultimately, CTA aims to provide full sky coverage from multiple observatory sites, using transparent access and identical tools to extract and analyse data.

The feasibility of the performance goals listed above is borne out by detailed simulations of arrays of telescopes, using currently available technology (details are given below). The implementation of CTA does requires significant advances in the engineering, construction and operation of the array, and the data access. These issues are addressed in the design study and the preparatory phase of CTA. Issues include:

Construction, installation and commissioning of the telescopes To reach the performance targets, tens of telescopes of 2-3 different types will be required, and the design of the telescopes must be optimised in terms of their construction cost, making best use of the economics of large-scale production. In current instruments, consisting at most of a handful of identical telescopes, design costs were a substantial fraction of total costs, enforcing a different balance between design and production costs. The design of the telescopes will have to concentrate on modularity and ease of installation and commissioning.

Reliability The reliability of current instruments is far from perfect, and downtimes of individual telescopes due to hardware or software problems are non-negligible. For CTA, telescope design and software must provide significantly improved reliability. Frequent down-times of individual telescopes in the array or of pixels within a telescope not only require substantial technical on-site support and cause higher operating costs, but in particular they make the data analysis much more complicated, requiring extensive simulations for each configuration of active telescopes, and inevitably result in systematic errors which are likely to limit the achievable sensitivity.

Operation scheduling and monitoring The large flexibility provided by the CTA array also raises new challenges concerning the scheduling of observations, taking into account the state of the array and the state of the atmosphere. For example, sky conditions may allow "discovery observations" in certain parts of the sky, but may prevent precise, deep observations of a source. Availability of a given telescope may be critical for certain types of observations, but may not matter at all in modes where the array is split up in many sub-arrays tracking different sources at somewhat reduced sensitivity. To make optimum use of the facility, novel scheduling algorithms will need to be developed, and the monitoring of the atmosphere over the full sky needs to be brought to a new level of precision. 
Data access So far, none of the current Cherenkov telescopes has made data publicly available, or has tools for efficient non-expert data access. Cherenkov telescopes are inherently more complicated than, say, X-ray satellite instruments in that they do not directly take images of the sky, but rather require extensive processing to go from the Cherenkov images to the parameters of the primary gamma ray. Depending on the emphasis in the data analysis-maximum detection rate, lowest energy threshold, best sensitivity, or highest angular resolution-there is a wide range of selection parameters, all resulting in different effective detection areas and instrument characteristics. Effective detection areas also depend on zenith angle, orientation relative to the Earth's magnetic field, etc. Background subtraction is critical in particular for extended sources which may cover a significant fraction of the field of view. Providing efficient data access and analysis tools represents a major challenge and requires significant lead times and extensive software prototyping and tests.

\section{Performance of Cherenkov Telescope Arrays}

In order to achieve improvements of a factor of 10 in several areas, it is essential to understand and review the factors limiting the performance, and to establish the extent to which limitations are of technical nature which can be overcome with sufficient effort (e.g. due to a given size of the camera pixels or point spread function (PSF) of the re ector), and to which extent they represent fundamental limitations of the technique (e.g. due to unavoidable fluctuations in the development of air showers).

To detect a cosmic gamma-ray source in a given energy band, three conditions have to be fulfilled:

The number of detected gamma rays $\mathbf{N}_{\gamma}$ has to exceed a minimum value, usually taken to be between 5 and 10 gamma rays. The number of gamma rays is the product of flux $\phi_{\gamma}$, effective detection area $A$, observing time $T$ (usually for sensitivity evaluation taken as between 25 and $50 \mathrm{~h}$ ) and a detection efficiency $\epsilon_{\gamma}$ which is typically not too far below unity. The number of detected gamma rays and hence the effective area $A$ are virtually always the limiting factor at the high-energy end of the useful energy range. For example, to detect a $1 \%$ Crab source above $100 \mathrm{TeV}$, which equivalent to a flux of $2 \times 10^{-16} \mathrm{~cm}^{-2} \mathrm{sec}^{-1}$, in $50 \mathrm{~h}$, an area $A$ of $\geq 30 \mathrm{~km}^{2}$ is required.

The statistical significance of the gamma ray excess has to exceed a certain number of standard deviations, usually taken to be 5. For background dominated observations of faint sources, significance can be approximated as $N_{\gamma} / \sqrt{N_{b g}}$ where the background events $N_{b g}$ arise from cosmic ray nuclei, cosmic ray electrons, local muons, or random images caused by nightsky background (NSB). Background events are usually distributed more or less uniformly across the useful field of view of the instrument. Their 
number is given by the flux per unit solid angle, $\phi_{b g}$, the solid angle $\Omega_{s r c}$ over which gamma rays from a candidate source (and hence background) are accumulated, the effective detection area $A_{b g}$, the observation time and a background rejection factor $\epsilon_{b g}$. The sensitivity limit $\phi_{\gamma}$ is hence proportional to $\sqrt{\epsilon_{b g} A_{b g} T \Omega_{s r c}} /\left(\epsilon_{\gamma} A_{\gamma} T\right) \sim \sqrt{\Omega_{s r c}} / \sqrt{\epsilon_{b g} A T}$ (assuming $A_{b g} \sim$ $\left.A_{\gamma}\right)$. In current instruments, electron and cosmic nucleon backgrounds limit the sensitivity in the medium to lower part of their energy range.

The systematic error on the number of excess gamma rays due to uncertainties in background estimates and background subtraction has to be sufficiently small, and has to be accounted for in the calculation of the significance. Fluctuations in the background rates due to changes in voltages, pulse shapes, calibration, in particular when non-uniform over the field of view, or in the cut efficiencies, e.g. due to non-uniform NSB noise, will result in such background systematics. Effectively, this means that a minimal signalto-background ratio is required to safely detect a source. The systematic limitation becomes important in the limit of small statistical errors, when event numbers are very large due to large detection areas, observation times, or low energy thresholds resulting in high count rates. Since both signal and background scale with $A$ and $T$, the systematic sensitivity limit is proportional to the relative background rate, $\phi_{\gamma} \sim\left(\epsilon_{b g} \Omega_{s r c}\right) / \epsilon_{\gamma}$. For current instruments, background uncertainties at a level of a few $\%$ have been reported [57]. High reliability and availability of telescopes and pixels as well as improved schemes for calibration and monitoring will be crucial in controlling systematic errors and exploiting the full sensitivity of the instrument. An accuracy of the background modelling and subtraction of $1 \%$ seems reasonable and is assumed in the following. Systematic errors may still limit sensitivity in the sub-100 GeV range.

Figure 4 illustrates the various sensitivity limitations in the context of a simple toy model. Obviously, sensitivity is boosted by large effective area $A$, efficient rejection of background, i.e. small $\epsilon_{b g}$, and in the case of pointlike structures by good angular resolution $\delta$ with $\Omega_{s r c} \propto \delta^{2}$. Sensitivity gains can furthermore be achieved with a large field of view of the instrument, observing multiple sources at a time and effectively multiplying the attainable observation time $T$.

The annual exposure time amounts to about $1,000 \mathrm{~h}$ of useful moonless observation time per year, varying by maybe $20 \%$ between good and excellent sites. Observations with partial moon may increase this by a factor of 1.5 , at the expense of reduced performance, depending on the amount of stray light. Some instruments, such as MAGIC, routinely operate under moonlight [58]. While in principle more than $500 \mathrm{~h}$ per year can be dedicated to a given source (depending on its RA, and the maximum zenith angle under which observations are carried out), in practice rarely more than $50 \mathrm{~h}$ to at most $100 \mathrm{~h}$ are dedicated to a given source per year. With the increased number of sources detectable for CTA, there will be pressure to reduce the time per source compared to current observations. 


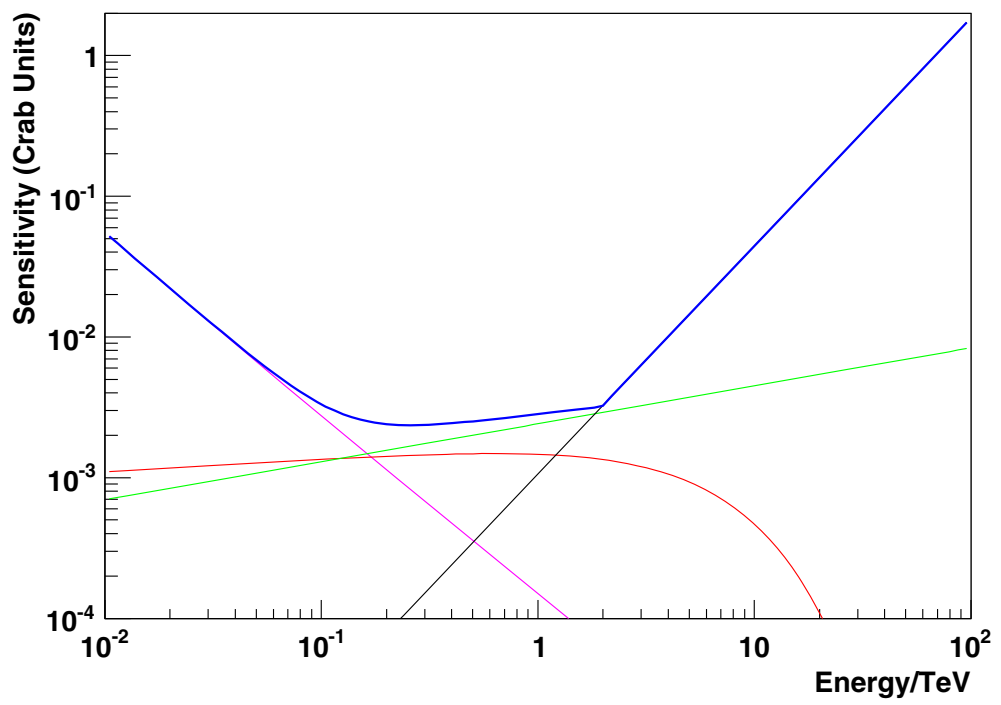

Fig. 4 Toy model of a telescope array to illustrate limiting sensitivity, quoted as the minimal detectable fraction of the Crab flux per energy band $\Delta \log _{10}(E)=0.2$ (assuming a simple power law for the Crab flux and ignoring the change in spectral index at low energy). The model assumes an energy-independent effective detection area of $1 \mathrm{~km}^{2}$, a gamma-ray efficiency of $\epsilon_{\gamma}$ of 0.5 , the same efficiency for detection of cosmic-ray electrons, a cosmic-ray efficiency after cuts of $\epsilon_{b g}=0.01$, an angular resolution $\delta$ of $0.1^{\circ}$ defining the integration region $\Omega_{s r c}$, and a systematic background uncertainty of $1 \%$. The model takes into account that cosmic-ray showers generate less Cherenkov light than gamma-ray showers, and are hence reconstructed at lower equivalent gamma-ray energy. At high energy, the sensitivity is limited by the gamma-ray count rate (black line), at intermediate energies by electron ( $r e d$ ) and cosmic-ray backgrounds (green), and at low energies, in the area of high statistics, by systematic background uncertainty (purple). The plot includes also the effect of the PSF improving like $1 / \sqrt{\mathrm{E}}$ (with PSF $=0.1^{\circ}$ for $80 \%$ containment at $200 \mathrm{GeV}$ )

In real systems, the effective area $A$, background rejection $\epsilon_{b g}$ and angular resolution $\delta$ depend on gamma-ray energy, since a minimal number of detected Cherenkov photons (around 50-100) are required to detect and analyse an image, and since the quality of shower reconstruction depends both on the statistics of detected photons and shower particles. The performance of the instrument depends on whether gamma-ray energies are in the sub-threshold regime, near the nominal energy threshold, or well above threshold.

In the sub-threshold regime, the amount of Cherenkov light is below the level needed for the trigger logic, at a sufficiently low rate of random triggers due to NSB photons. Only showers with upward fluctuations in the amount of Cherenkov light will occasionally trigger the system. At GeV energies these fluctuations are large and there is no sharp trigger threshold. Energy measurement in this domain is strongly biased.

In the threshold regime, there is usually enough Cherenkov light for triggering the system but the signal in each telescope may still be too low for (a) location of the image centroid, (b) determination of the direction of the image major axis, or (c) accurate energy assignment. Frequently, a higher threshold 
than that given by the trigger is imposed in the data analysis. Most showers with upward fluctuations will be reconstructed in a narrow energy range at the trigger (or analysis) threshold. Sources with cut-offs below the analysis threshold may be detectable but only at very high flux levels. Good imaging and spectroscopic performance of the instrument is only available at energies $\geq 1.5 \times$ the trigger threshold.

High sensitivity over a wide energy range, therefore, requires an instrument which is able to detect a sufficient number of Cherenkov photons for low energy showers, which covers a very large area for high-energy showers, and which provides high angular resolution and background rejection. High angular resolution is also crucial to resolve fine structures in extended sources such as supernova remnants. On the other hand, for the detection of extended sources, the integration region $\Omega_{s r c}$ is determined by the source size rather than the angular resolution and cosmic-ray rejection becomes a most critical parameter in minimising statistical and systematic uncertainties.

A crucial question is therefore to which extent angular resolution and cosmic-ray rejection can be influenced by the design of the instrument, by parameters such as the number of Cherenkov photons detected or the size of the photo-sensor pixels. Simulation studies assuming an ideal instrument [59], one which detects all Cherenkov photons reaching the ground with perfect resolution for impact point and photon direction, show that achievable resolution and background rejection are ultimately limited by fluctuations in the shower development. Angular resolution is in addition influenced by the deflection of shower particles in the Earth's magnetic field, making the reconstructed shower direction dependent on the energy sharing between electron and positron in the first conversion of a gamma ray (Fig. 5). However, these resolution limits (Fig. 6) are well below the resolution achieved by current instruments. At $1 \mathrm{TeV}$, a resolution below one arc-minute is in principle achievable. Similar conclusions appear to hold for cosmic-ray background rejection. There is a virtually irreducible background due to events in which, in the first interaction of a cosmic ray, almost all the energy is transferred to one or a few neutral pions and, therefore, to electromagnetic cascades (see, e.g. [60]). However, with their typical cosmic-ray rejection factors of $>10^{3}$ at $\mathrm{TeV}$ energies, current instruments still seem 1-2 orders of magnitude away from this

Fig. 5 Two low-energy gamma-ray showers developing in the atmosphere. Both gamma rays were incident vertically. The difference in shower direction results from the energy sharing between electron and positron in the first conversion and the subsequent deflection in the Earth's magnetic field
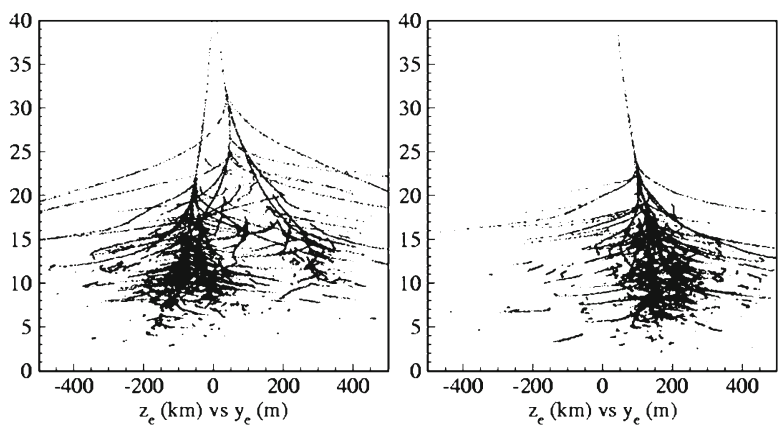


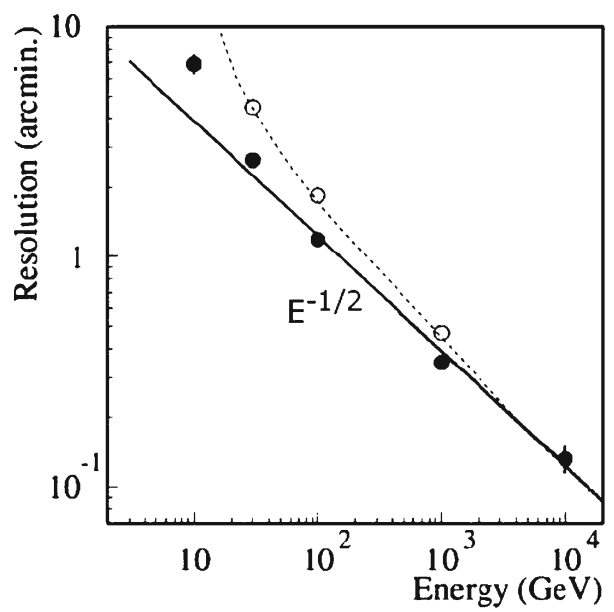

Fig. 6 Limiting angular resolution of Cherenkov instruments as a function of gamma-ray energy, derived from a likelihood fit to the directions of all Cherenkov photons reaching the ground, and assuming perfect measurement of photon impact point and direction. At low energies, the resolutions differ in the bending plane of the Earth's magnetic field (open symbols) and in the orthogonal direction (closed symbols). The simulations assume near-vertical incidence at the H.E.S.S. site in Namibia

limit, offering space for improvement. Such improvements could result from improved imaging of the air shower, both in terms of resolution and photon statistics, and from using a large and sensitive array to veto cosmic-ray induced showers based on the debris frequently emitted at relatively large angles to the shower axis.

At low energies, cosmic-ray electrons become the dominant background, due to their steep spectrum. Electrons and gamma-rays cannot be distinguished efficiently using shower characteristics, as both induce electromagnetic cascades. The height of the shower maximum differs by about one radiation length [61], but this height also fluctuates from shower to shower by about one radiation length, rendering an efficient rejection impossible. A technique which is beyond the capability of current instruments but might become possible with future arrays is to detect Cherenkov radiation from the primary charged particle and use it as a veto [59]. Detection of the "direct Cherenkov light" has been proposed [54] and successfully applied [62] for highly charged primary nuclei such as iron, where Cherenkov radiation is enhanced by a factor of $Z^{2}$. While in a $100 \mathrm{~m}^{2}$ telescope, an iron nucleus generates $\mathrm{O}(1000)$ detected photons, a charge- 1 primary will provide at most a few photons, not far from night sky noise levels. Larger telescopes, possibly with improved photo-sensors, fine pixels and high temporal resolution, could enable detection of primary Cherenkov light from electrons, at the expense of gamma-ray efficiency, since gamma-rays converting at high altitude will be rejected, too, and since unrelated nearby cosmic rays may generate fake vetos. Nevertheless, this approach (not yet studied in detail) may help at the lowest energies where event numbers are high but there are large uncertainties in the background 
systematics. Sakahian et al. [63] note that at energies $<20 \mathrm{GeV}$, deflection of electrons in the Earth's magnetic field is sufficiently large to disperse Cherenkov photons over a larger area on the ground, reducing light density and therefore the electron-induced trigger rate. The effect is further enhanced by a dispersion in photon arrival times.

In summary, it is clear that the performance of Cherenkov telescope arrays can be improved significantly, before fundamental limitations are reached.

\section{The Cherenkov Telescope Array}

The CTA consortium plans to operate from one site in the southern and one in the northern hemisphere, allowing full-sky coverage. The southern site will cover the central part of the galactic plane and see most of the galactic sources and will therefore be designed to have sensitivity over the full energy range. The northern site will be optimised for extragalactic astronomy, and will not require coverage of the highest energies.

Determining the arrangement and characteristics of the CTA telescopes in the two arrays is a complex optimisation problem, balancing cost against performance in different bands of the spectrum. This section will address the general criteria and considerations for this optimisation, while the technical implementation is covered in the following sections.

\subsection{Array layout}

Given the wide energy range to be covered, a uniform array of identical telescopes, with fixed spacing, is not the most efficient solution for the CTA. For the purpose of discussion, separation into three energy ranges, without sharp boundaries, is appropriate:

The low-energy range $\leq \mathbf{1 0 0} \mathbf{~ G e V}$ To detect showers down to a few tens of $\mathrm{GeV}$, the Cherenkov light needs to be sampled and detected efficiently, with the fraction of area covered by light collectors being of the order of $10 \%$ (assuming conventional PMT light sensors). Since event rates are high and systematic background uncertainties are likely to limit the achievable sensitivity, the area of this part of the array can be relatively small, being of order of a few $10^{4} \mathrm{~m}^{2}$. Efficient photon detection can be achieved either with few large telescopes or many telescopes of modest size. For very large telescopes, the cost of the dish structures dominates, for small telescopes the photon detectors and electronics account for the bulk of the cost. A (shallow) cost optimum in terms of cost per telescope area is usually reached for medium-sized telescopes in the 10-15 m diameter range. However, if small to medium-sized telescopes are used in this energy range, the challenge is to trigger the array, since no individual telescope detects enough Cherenkov photons to provide a reliable trigger 
signal. Trigger systems which combine and superimpose images at the pixel level in real time, with a time resolution of a few ns, can address this issue [64] but represent a significant challenge, given that a single 1,000pixel telescope sampled at (only) $200 \mathrm{MHz}$ and 8 bits per pixel generates a data stream of more than one Tb/s. CTA designs conservatively assume a small number of very large telescopes, typically with about a $20-30 \mathrm{~m}$ dish diameter, to cover the low energy range.

The core energy range from about $100 \mathrm{GeV}$ to about $10 \mathrm{TeV}$ shower detection and reconstruction in this energy range are well understood from current instruments, and an appropriate solution seems a grid of telescopes of the $10-15 \mathrm{~m}$ class, with a spacing of about $100 \mathrm{~m}$. Improved sensitivity is obtained both by the increased area covered, and by the higher quality of shower reconstruction, since showers are typically imaged by a larger number of telescopes than is the case for current few-telescope arrays. For the first time, array sizes will be larger than the Cherenkov light pool, ensuring that images will be uniformly sampled across the light pool, and that a number of images are recorded close to the optimum distance from the shower axis (about 70-150 m), where the light intensity is large and intensity fluctuations are small, and where the shower axis is viewed under a sufficiently large angle for efficient reconstruction of its direction. At H.E.S.S. for example, events which are seen and triggered by all four telescopes provide significantly improved resolution and strongly reduced backgrounds, but represent only a relatively small fraction of events. Unless energies are well above trigger threshold, only events with shower core locations within the telescope square can trigger all telescopes. A further advantage is that an extended telescope grid operated with a two-telescope trigger condition will have a lower threshold than a small array, since there are always telescopes sufficiently close to the shower core.

The high-energy range above $10 \mathrm{TeV}$ Here, the key limitation is the number of detected gamma-ray showers and the array needs to cover multi- $\mathrm{km}^{2}$ areas. At high energies the light yield is large, so showers can be detected well beyond the $150-\mathrm{m}$ radius of a typical Cherenkov light pool. Two implementation options can be considered: either a large number of small telescopes with mirror areas of a few $\mathrm{m}^{2}$ and spacing matched to the size of the light pool of 100-200 m, or a smaller number of larger telescopes with some $10 \mathrm{~m}^{2}$ area which can see showers up to distance of $\geq 500 \mathrm{~m}$, and can hence be deployed with a spacing of several $100 \mathrm{~m}$, or in widely separated subclusters of a few telescopes. While it is not immediately obvious which options offers best cost/performance ratio at high energies, the subcluster concept with larger telescopes has the advantage of providing additional high-quality shower detection towards lower energies, for impact points near the subcluster.

Figure 7 shows possible geometries of arrays with separate regions optimized for low, intermediate and high energies. 
Fig. 7 A quadrant of possible array schemes promising excellent sensitivity over an extended energy range, as suggested by the Monte Carlo studies. The centre of the installation is near the upper left corner. Telescope diameters are not drawn to scale. In the upper right part, clusters of telescopes of the 12-m class are shown at the perimeter, while in the lower left part an option with wide-angle telescopes of the 3-4 m class is shown

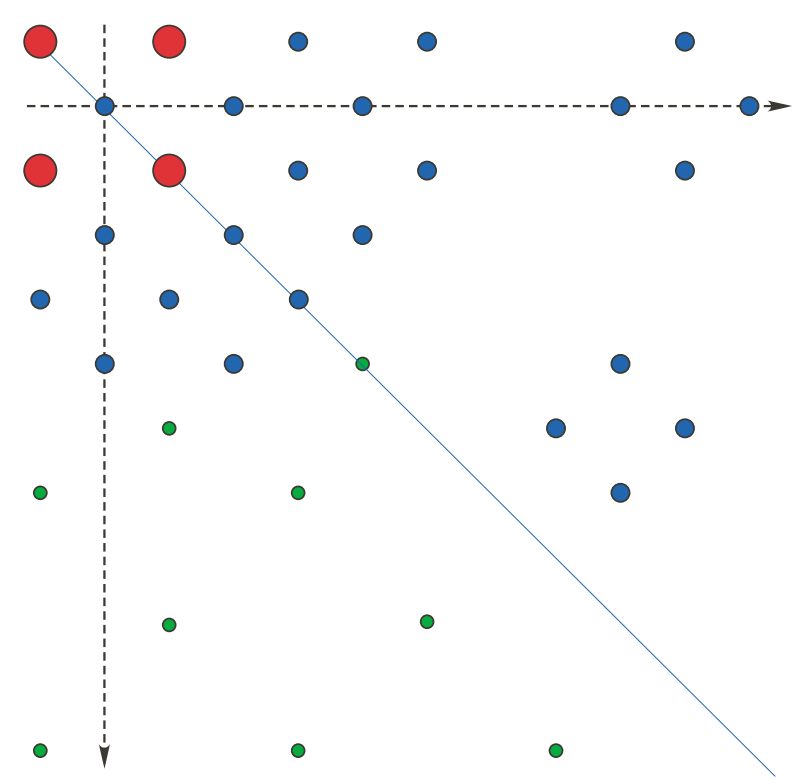

\subsection{Telescope layout}

Irrespective of the technical implementation details, as far as its performance is concerned, a Cherenkov telescope is primarily characterised by its light collection capability, i.e. the product of mirror area, photon collection efficiently and photon detection efficiency, by its field of view and by its pixel size, which limits the size of image features which can be resolved. The optical system of the telescope should obviously be able at achieve a point spread function matched to the pixel size. The electronics for signal capture and triggering should provide a bandwidth matched to the length of Cherenkov pulses of a few nanoseconds. The performance of an array is also dependent on the triggering strategy; Cherenkov emission from air showers has to be separated in real time from the high flux of night sky background photons, based on individual images and global array information. The huge data stream from Cherenkov telescopes does not allow untriggered recording.

The required light collection capability in the different parts of the array is determined by the energy thresholds, as outlined in the previous section. In the following, field of view, pixel size and the requirements on the readout system and trigger system are reviewed.

\subsubsection{Field of view}

Besides mirror area, an important telescope design parameter is the field of view. A relatively large field of view is mandatory for the widely spaced 
telescopes of the high-energy array, since the distance of the image from the camera centre scales with the distance of the impact point of the air shower from the telescope. For the low- and intermediate-energy arrays, the best choice of the field of view is not trivial to determine. From the science point of view, large fields of view are highly desirable, since they allow:

- the detection of high-energy showers at large impact distance without image truncation;

- the efficient study of extended sources and of diffuse emission regions; and

- large-scale surveys of the sky and the parallel study of many clustered sources, e.g. in the band of the Milky Way.

In addition, a larger field of view generally helps in improving the uniformity of the camera and reducing background systematics.

However, larger fields of view for a given pixel size, result in rapidly growing numbers of photo-sensor pixels and electronics channels. Large fields of view also require technically challenging telescope optics. With the current singlemirror optics and $f / d$ ratios in the range up to 1.2 , an acceptable point spread function is obtained out to $4-5^{\circ}$. Larger fields of view with single-mirror telescopes require increased $\mathrm{f} / \mathrm{d}$ ratios, in excess of 2 for a $10^{\circ}$ field of view (see Fig. 8, [65]), which are mechanically difficult to realise, since a large and heavy focus box needs to be supported at a long distance from the dish. Also, the single-mirror optics solutions which provide the best imaging use DaviesCotton or elliptical dish geometries, which in turn result in a time dispersion of shower photons which seriously impacts on the trigger performance once dish diameters exceed $15 \mathrm{~m}$. An alternative solution is the use of secondary mirrors. Using non-spherical primaries and secondaries, good imaging over fields of up to $10^{\circ}$ diameter can be achieved [66]. Disadvantages are the increased cost and



Fig. 8 Focal ratio required for sufficiently precise shower imaging, as a function of the half angle of the field of view [65]. Points: simulations for spherical design (green), parabolic design with constant radii (red), Davies-Cotton design (violet), parabolic design with adjusted radii (blue). Lines: third-order approximation for a single-piece paraboloid (red) and a single-piece sphere (green) 
complexity, significant shadowing of the primary mirror by the secondary, and complex alignment issues if faceted primary and secondary mirrors are used. With the resulting large range of incidence angles of photons onto the camera, can imply that baffling of albedo also becomes an issue.

The choice of the field of view therefore requires that the science gains and the cost and increased complexity be carefully balanced. When searching for unknown source types which are not associated with non-thermal processes in other, well-surveyed wavelength domains, a large field of view helps, as several sources may appear in typical fields of view. This increases the effective observation time per source by a corresponding factor compared to an instrument which can look only at one source at a time. An instrument with CTA-like sensitivity is expected to detect of the order of 1,000 sources. In the essentially one-dimensional galactic plane, there will always be multiple sources in a field of view. In extragalactic space, the average angular distance between (an estimated 500) sources would be about $10^{\circ}$, implying that even for the maximum conceivable fields of view the gain is modest. Even in the galactic plane, a very large field of view will not be the most cost-effective solution, since the gain in terms of the number of sources viewed simultaneously scales essentially with the diameter of the field of view, given that sources are likely to cluster within a fraction of a degree from the plane, whereas camera costs scale with the diameter squared. A very rough estimate based on typical dish costs and per-channel pixel and readout costs suggests an economic optimum in the cost per source-hour at around a diameter of 6-8 field of view.

The final choice of the field of view will have to await detailed studies related to dish and mirror technology and costs, and the per-channel cost of the detection system.

Sensitivity estimates given below do not include an enhancement factor accounting for multiple sources in the field of view, but effective exposure time should increase by factors of $\geq 4$ for Galactic sources, and sensitivity correspondingly by factors of $\geq 2$.

\subsubsection{Pixel size}

The size of focal plane pixels is another parameter which requires careful optimisation. Figure 9 illustrates how a shower image is resolved at pixel sizes ranging from $0.28^{\circ}$ (roughly the pixel size of the HEGRA telescopes) down to pixel sizes of $0.07^{\circ}$, as used for example in the large H.E.S.S. II telescope. The cost of focal plane instrumentation is currently driven primarily by the number of pixels and, therefore, scales like the square of the inverse pixel size. The gain due to the use of small pixels depends strongly on the analysis technique. In the classical second-moment analysis, performance seems to saturate for pixels smaller than $0.2-0.15^{\circ}$ [67]. Analysis techniques which use the full image distribution (e.g. [68]), on the other hand, can extract the information contained in the well-collimated head part of high-intensity images, as compared to the more diffuse tail, and benefit from pixel sizes as small as $0.06-0.03^{\circ}[59,66]$. Pixel size also influences trigger strategies. 

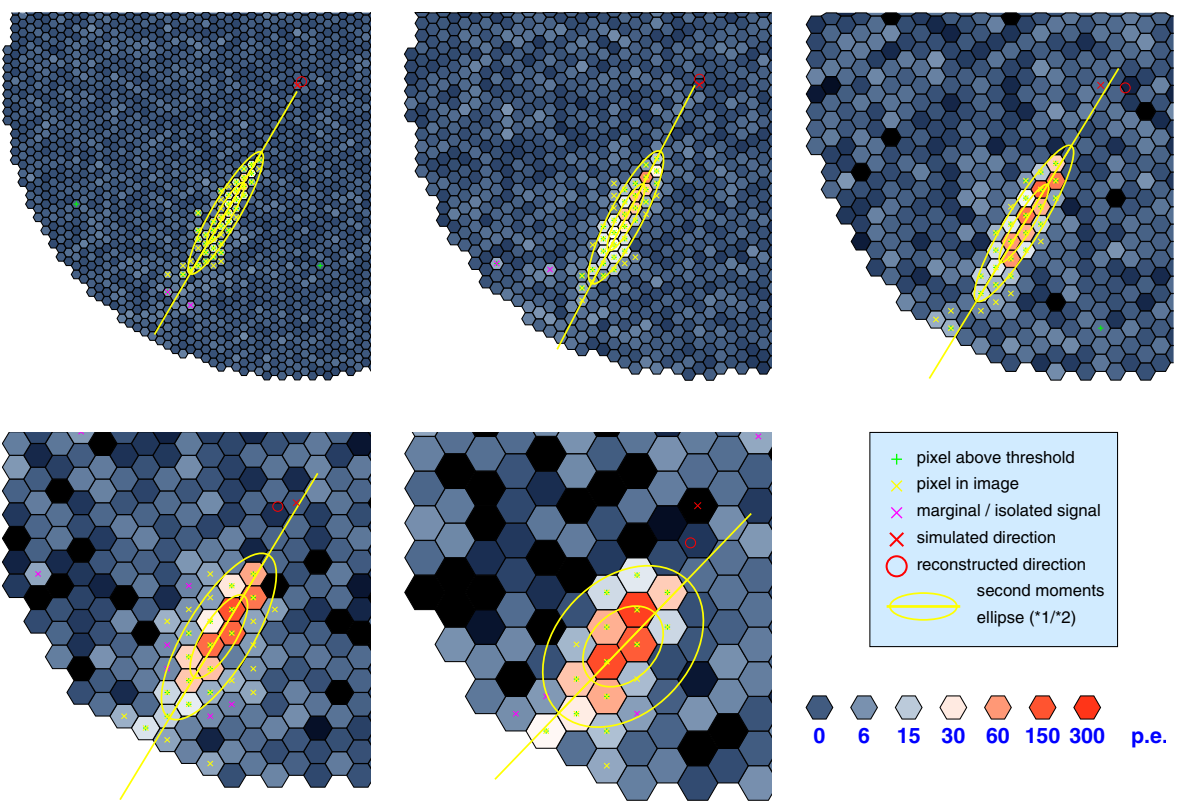

Fig. 9 Part of the field of view of cameras with different pixel sizes $(0.07,0.10,0.14,0.20$, and $0.28^{\circ}$ ) but identical field-of-view (of about $6^{\circ}$ ), viewing the same shower (460 GeV gamma-ray at $190 \mathrm{~m}$ core distance) with a $420 \mathrm{~m}^{2}$ telescope. Low-energy showers would be difficult to register, both with very small pixels (signal not contiguous in adjacent pixels) and with very large pixels (not enough pixels triggered above the increased thresholds, due to high NSB rates)

For large pixels, gamma-ray images are contiguous, allowing straight-forward topological triggers, whereas for small pixels, low-energy gamma-ray images may have gaps between triggered pixels.

The final decision concerning pixel size (and telescope field of view) will to a significant extent be driven by the cost per pixel. Current simulations favour pixel sizes of $0.07-0.1^{\circ}$ for the large telescopes, allowing the resolution of compact low-energy images and reducing the rate of NSB photons in each pixel, $0.15-0.2^{\circ}$ for the medium size telescopes, similar to the pixel sizes used by H.E.S.S. and VERITAS, and $0.2-0.3^{\circ}$ for the pixels of the telescopes in the halo of the array, where large fields of view are required but shower images also tend to be long due to the large impact distances and the resulting viewing angles. Studies to determine the benefits of smaller pixels, as are proposed for AGIS-type dual-mirror telescopes (http://tmva.sourceforge.net), are underway for the medium-sized telescopes.

\subsubsection{Signal recording}

Most modern telescopes use some kind of transient recorders to capture pixel signals, either with analogue switched-capacitor systems or with fast digitisers [69], so that, at least in principle, signal shape and timing can be used in 
the image analysis. Signal shape and timing can be employed in two ways: (a) to reject backgrounds such as hadronic showers and local muons; and (b) to reduce the signal integration windows and hence the amount of NSB noise in the shower image. For example, muon rejection based on signal waveform is discussed in [70]. Quantifying how much background rejection can be improved using these techniques is non-trivial. The effect of signal-shape image selection is correlated with other cuts imposed in the analysis. For single telescopes, signal shape and timing can provide significant improvements. For telescope systems, the cuts on image shapes in multiple telescopes are already very powerful and background events passing these cuts will have images and signal shapes that look very much like those of gamma-rays, so that less improvement is expected, if any. The second area where signal waveform recording can improve performance concerns the signal amplitudes. In particular for larger shower impact parameters, photon arrival times are not isochronous across the image (Fig. 10), and photons in the "tail" end of the image arrive with significant delays compared to those from its "head". Use of variable and matched integration windows across the image allows the extraction of shower signals with minimal contamination from NSB noise. Use of signal shape and timing information is already used in the current MAGIC [71] and VERITAS systems, and these results will help to guide final design choices for CTA.
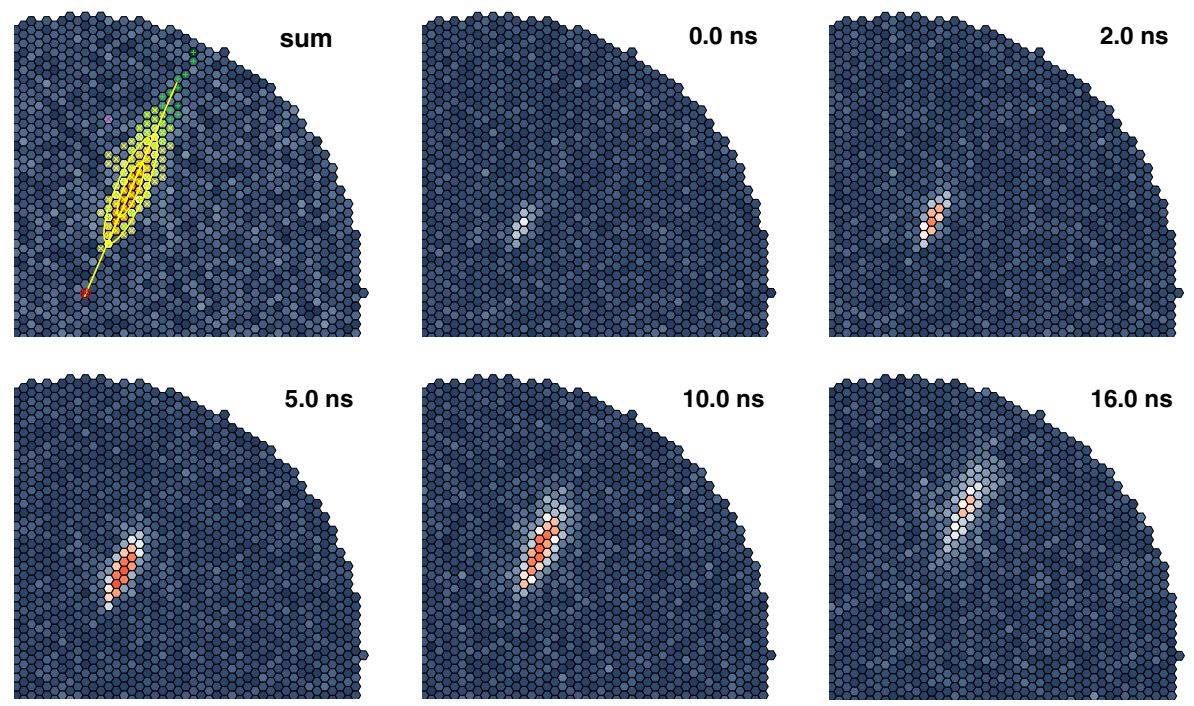

Fig. 10 Integrated signal (upper left) and 1 ns samples of the development of a $10 \mathrm{TeV}$ gamma shower at $250 \mathrm{~m}$ core distance as seen in a telescope with optics and pixels similar to a H.E.S.S.-1 telescope but with a FoV of $10^{\circ}$ diameter. Pixels near the "head" of the shower have a pulse width dominated by the single photoelectron pulse width, while those in the "tail" of the shower see longer pulses. The shower image moves across almost half the FoV in about $25 \mathrm{~ns}$ 
The performance numbers quoted for the simulations described below are conservative in that they are based on fixed (and relatively large) signal integration windows. Improvements can be expected once the use of image shape information is fully understood.

\subsubsection{Trigger}

The trigger scheme and readout electronics are closely related and fundamentally influence the design and performance of the telescope array. For most applications, multi-telescope trigger coincidence is required to reject backgrounds at the trigger level and to reduce the load on the data acquisition system. The main issue here is how much information is exchanged between telescopes, and how image information is stored while the trigger decision is made.

One extreme scenario is to let each telescope trigger independently and only exchange a trigger flag with neighbouring telescopes, allowing identification of coincident triggers (e.g. [72]). The energy threshold of the system is then determined by the minimum threshold at which a telescope can trigger. The other extreme is to combine signals from different telescopes at the pixel level, either in analogue or digital form, and to extract common image features. In this case, the system energy threshold could be well below the thresholds of individual telescopes, which is important when the array is made up of many small or medium-sized telescopes. However, the technical complexity of such a solution is significant. There is a wide range of intermediate solutions, where trigger pre-processors extract image features, such as the image centroid, on a telescope basis and the system trigger decision includes this information.

In cases where individual telescopes generate a local trigger, pixel signals need to be stored while a global trigger decision is made. The time for which signals can be stored without introducing deadtime, is typically $\mathrm{ms}$ in the case of digital storage and $\mu \mathrm{s}$ if analogue storage is used, which strongly influences the design of higher level triggers.

Trigger topology is another important issue. Triggers can either be derived locally within the array by some trigger logic connecting neighbouring telescopes, or all trigger information can be routed to a central station where a global decision is made, which is then propagated back to the telescopes. The first approach requires shorter signal storage at the telescopes and is more easily scaled up to large arrays, the second provides maximum flexibility. Whether local or global, trigger schemes will employ a multi-level hierarchy, with a first trigger level acting on pixels and pixel groups, and higher levels using information on image topology and/or the topology of triggered telescopes in the array. As in modern high-energy physics experiments, trigger decisions will, to the extent possible, be performed using programmable rather than "hardwired" processors. If the signal is recorded using fast digitisers, even the first-level discrimination of pixel signals could be implemented digitally in the gate array controlling the digitiser, instead of applying analogue thresholds. 
Whatever implementation is chosen, it is important that the trigger system is very flexible and software-configurable, since operation modes vary from deep observations, where all telescopes follow the same source, to monitoring or survey applications, where groups of a few telescopes or even single telescopes point in different directions.

The simulations discussed below assume a very conservative approach. Each telescope makes an independent trigger decision with thresholds defined such that the telescope trigger rate is in the manageable range of a few to some tens of $\mathrm{kHz}$. This is followed by a global decision based on the number of triggered telescopes.

\subsection{CTA performance summary}

Section 8 gives a detailed description of the layout and performance studies conducted so far for CTA. Many candidate layouts have been considered. Here we provide a brief description of the nature and performance of one promising configuration (E), which is illustrated in Fig. 18. This configuration utilises three telescope types: four $24 \mathrm{~m}$ telescopes with $5^{\circ}$ field-of-view and $0.09^{\circ}$ pixels, 23 telescopes of $12 \mathrm{~m}$ diameter with $8^{\circ}$ field-of-view and $0.18^{\circ}$ pixels, and 32 telescopes of $7 \mathrm{~m}$ diameter with a $10^{\circ}$ field-of-view and $0.25^{\circ}$ pixels. The telescopes are distributed over $\sim 3 \mathrm{~km}^{2}$ on the ground and the effective collection area of the array is considerably larger than this at energies beyond $10 \mathrm{TeV}$. The sensitivity of array $\mathrm{E}$ from detailed calculations and using standard data analysis techniques is shown in Fig. 23. More sophisticated analyses result in sensitivities that are $\sim 20 \%$ better across the whole energy range. As Fig. 23 shows, such an array performs an order of magnitude better than an instrument like H.E.S.S. over most of required energy range. Figure 25 shows the angular resolution of this array, which approaches one arcminute at high energies. The energy resolution of layout $\mathrm{E}$ is better than $10 \%$ above a few hundred GeV.

Array layout $\mathrm{E}$ has a nominal construction cost of $80 \mathrm{M} €$ and meets the main design goals of CTA. Given that the configuration itself, and the analysis methods used, have not yet been optimised, it is likely that a significantly better sensitivity can be achieved with an array of this nominal cost which follows the same basic concept. Therefore, despite the uncertainties in the cost model employed (see Section 7.5), we are confident that the design goals of CTA can be realised at close to the envisaged cost.

\section{Realizing CTA}

This section provides a brief overview of the position of CTA in the European and global context, the organisation of CTA during the various stages, of its operation as an open observatory, of the potential sites envisaged for CTA, and of the schedule for and cost of CTA design, construction and operation. 
7.1 CTA and the European strategy in astrophysics and astroparticle physics

CTA, as a major future facility for astroparticle physics, is firmly embedded in the European processes guiding science in the fields of astronomy and astroparticle physics.

The European Strategy Forum on Research Infrastructures (ESFRI) ESFRI is a strategic organisation whose objective is to promote the scientific integration of Europe, to strengthen the European Research Area and to increase its international impact. A first Roadmap for pan-European research infrastructures was released in 2006, listing CTA as an "emerging project". In the December 2008 update of this Roadmap, CTA was included as one of eight Physical Sciences and Engineering projects, together with facilities such as E-ELT, KM3Net and SKA. As such, CTA is eligible for FP7 Preparatory Phase funding. The CTA application for this funding was successful, providing up to $5.2 \mathrm{M} €$ for the preparation of the construction of the observatory in 3 years time. The contracts with the EC are in the process of being finalised and signed.

The Astroparticle Physics European Coordination (ApPEC) group ApPEC was created to enhance coordination in astroparticle physics across Europe. It has stimulated cooperation and convergence between competing groups in Europe, and has initiated the production of a European roadmap in astroparticle physics, on which CTA is one of the key projects.

ASPERA ASPERA is a network of national government agencies responsible for coordinating and funding national research efforts in Astroparticle Physics. One of the tasks of ASPERA is to create a scientific roadmap for Astroparticle Physics (http://www.aspera-eu.org/images/stories/roadmap/ aspera_roadmap.pdf) and link it with the more general European scientific infrastructure roadmap. A Phase I roadmap has been published, presenting the overarching science questions and the new instruments planned to address these questions. Phase II saw the release of the resulting "European Strategy for Astroparticle Physics" in September 2008, prioritising the projects under consideration. In this roadmap, CTA emerges as a near-term high-priority project. The roadmap states:

The priority project for VHE gamma-ray astrophysics is the Cherenkov Telescope Array, CTA. We recommend design and prototyping of CTA, the selection of sites, and proceeding rapidly towards start of deployment in 2012. CTA was one of the two projects targeted by the 2009 ASPERA Common Call for cross-national funding and received in total $2.7 \mathrm{M} €$ from national funding agencies.

The ASTRONET Eranet ASTRONET was created by a group of European funding agencies to establish comprehensive long-term planning for the development of European astronomy. The objective of this effort is to consolidate and reinforce the world-leading position that European astronomy attained at the beginning of this century. Late in 2008, ASTRONET released "The ASTRONET Infrastructure Roadmap: A Strategic Plan for 
European Astronomy". CTA is one of the three medium-scale facilities recommended on this roadmap, together with the neutrino telescope KM3Net and the solar telescope EST.

\subsection{CTA in the world-wide context}

Ground-based gamma-ray astronomy has attracted considerable attention world-wide, and while CTA is the key project in Europe, other projects have been considered elsewhere. These include primarily:

The Advanced Gamma-ray Imaging System (AGIS) In both science an instrumentation, AGIS (http://www.agis-observatory.org/) followed a very similar plan to that of CTA. The AGIS project was presented in a White Paper prepared for the Division of Astrophysics of the American Physical Society [8]. AGIS proposed a square-kilometre array of mid-sized telescopes, similar to the core array of mid-sized telescopes in CTA but without the additional large telescopes to cover the very lowest energies, and an extended array of small telescopes to provide large detection area at the very highest energies. The baseline configuration of AGIS consisted of 36 two-mirror Schwarzschild-Couder telescopes with an $11.5 \mathrm{~m}$ diameter primary mirror. These have a large field of view and a very good angular resolution. Close contacts were established between AGIS and CTA, during the design study phase; information was openly exchanged and common developments undertaken. After a US review panel recommended that AGIS join forces with CTA, the US members of the AGIS Collaboration have joined CTA in spring 2010. Within the overall context of CTA, development of Schwarzschild-Couder telescopes will be continued to investigate their potential for further improving CTA performance. Significant intellectual, technological and financial contributions to CTA from the US groups are anticipated. Strong US participation in CTA was endorsed by PASAG and the Decadal Survey in Astronomy and Astrophysics (Astro-2010).

\section{The High-Altitude Water-Cherenkov Experiment (HAWC) HAWC} (http://hawc.umd.edu/) builds on the technique developed by the MILAGRO group, which detects shower particles on the ground using water Cherenkov detectors, and reconstructs the shower direction using timing information. It is proposed to construct the new detector on a site at 4,100 m a.s.l. in the Sierra Negra, Mexico. HAWC will provide a tenfold increase in sensitivity over MILAGRO and detection capability down to the lower energy of $100 \mathrm{GeV}$, largely due to its increased altitude. While it will have lower sensitivity, poorer angular resolution and a higher energy threshold compared to CTA, HAWC has the advantage of a large field of view $(\approx 2 \pi$ sr $)$ and nearly $100 \%$ duty cycle. HAWC therefore complements imaging Cherenkov instruments. In fact, it would

\footnotetext{
${ }^{4}$ Particle Astrophysics Scientific Assessment Group of the High Energy Physics Advisory Panel
} 
be desirable to construct and operate a similar instrument in the southern hemisphere, co-located with CTA.

The Large High Altitude Air Shower Observatory (LHAASO) LHAASO is an extensive $\left(\mathrm{km}^{2}\right)$ cosmic ray experiment. The proposal is to locate this near the site of the ARGO and AS-Gamma experiments in Tibet, at 4,300 $\mathrm{m}$ a.s.l. The array includes large-scale water Cherenkov detectors $\left(90,000 \mathrm{~m}^{2}\right)$, ground scintillation counter arrays for detecting both muons and electromagnetic particles, fluorescence/Cherenkov telescope arrays and a shower core detector array. The science goals encompass a survey of gamma-ray sources in the energy range $\geq 100 \mathrm{GeV}$, measurement of gamma-ray energy spectra of sources above $30 \mathrm{TeV}$ to identify cosmic ray sources, and the measurement of cosmic ray spectra and composition at energies above $30 \mathrm{TeV}$. If realised, LHASSO will complement the northern CTA array, as it concentrates primarily on the detection of lowenergy gamma-rays in the energy range from a few times $10 \mathrm{GeV}$ to some $100 \mathrm{GeV}$.

In summary, the other large-scale instruments for ground-based gamma-ray astronomy that are being discussed outside Europe (e.g. HAWC, LHAASO), are complementary to CTA in their capabilities.

\subsection{Operation of CTA as an open observatory}

CTA is to address a wide range of astroparticle physics and astrophysics questions. The majority of studies will be based on observations of specific astronomical sources. The scientific programme will hence be steered by proposals to conduct measurements of specific objects. CTA will be operated as an open observatory. Beyond a base programme, which will include for example a survey of the Galaxy and deep observations of "legacy sources", observations will be conducted according to observing proposals selected for scientific excellence by peer-review among suggestions received from the community. Following the general procedures developed for and by other major astrophysical facilities, a substantial number of outstanding proposals from scientists working in institutions outside the CTA-supporting countries will be executed. All data obtained by the CTA will be made available in an archive that is accessible to scientists outside the proposing team.

Following the experience of currently operating Cherenkov telescope observatories, the actual observations will normally be conducted over an extended period in time, with several different projects being scheduled each night. The operation of the array will be fairly complex. CTA observations will not, therefore, be conducted by the scientists whose individual proposals were selected, but by a dedicated team of operators.

CTA observatory operation involves proposal handling and evaluation, managing observation and data-flow, and maintenance. The actual work may be conducted in a central location or in decentralised units (e.g. a data centre and an operations centre) with a coordinating office. 
Fig. 11 Work flow diagram of the CTA observatory. The three main elements which guarantee the functionalities of the observatory are the Science Operation Centre, the Array Operation Centre and the Data Centre. Data handling and dissemination will build on existing infrastructures, such as EGEE and GÉANT



\subsubsection{Observatory logistics}

The main logistic elements of the CTA observatory are: the Science Operation Centre (SOC), which is in charge of the organisation of observations; the Array Operation Centre (AOC), which looks after the operation and monitoring of the telescopes, and the Science Data Centre (SDC), which provides and disseminates data and analysis software to the science community at large, and using the standards of the International Virtual Observatory Alliance (see Fig. 11).

The use of existing infrastructures, such as EGEE and GÉANT, and the use of a Virtual Observatory is recommended for all data management tasks in the three elements of the CTA observatory. The high data rate of CTA, together with the large computing power required for data analysis, demand dedicated resources. Hence, EGEE-Grid infrastructures and middleware for distributed data storage, analysis and data access are considered the most efficient solution for CTA. The CTA observatories will very probably be placed in remote locations in southern Africa, Latin or Central America, and/or the Canary Islands. Thus, high-bandwidth networking is critical for remote diagnostics and instant transfer of the data to well-connected European data centres. As for other projects in astronomy, a CTA Virtual Organisation, will provide access to the data. CTA aims to support a wide scientific community, providing access to all levels of data that is archived in a standardised way.

It is envisaged to start CTA operations already during the construction phase as soon as the first telescopes are ready to conduct competitive science operations.

\subsubsection{Proposal handling}

The world-wide community of scientists actively exploiting the results from ground-based VHE gamma-ray experiments currently consists of about 600 physicists (about 150 in each of the H.E.S.S. and MAGIC Collaborations, 
about 100 in VERITAS, 50 in Cangaroo and 50 in Indian gamma ray activities, plus about 100 scientists either associated, or regularly collaborating, with these experiments). Planning and designing CTA involves about another 100 scientists not currently participating in either of the currently running experiments. Proposals for observations with CTA are hence expected to serve a community of at least 700 scientists, larger than that of any national astronomical facility in Europe, and comparable to the size of the community using the ESO observatory in the 1980s. CTA must therefore efficiently deal with a large number of proposals for a facility which, based on experience with current experiments, is expected to be oversubscribed by a large factor. CTA plans to follow the practice of other major, successful observatories (e.g. ESO), and announce calls for proposals at regular intervals. These proposals will be peer-reviewed by a group of international experts which will change on a regular basis. Different classes of proposals (targeted, surveys, time-critical, target of opportunity, and regular programmes) are foreseen, as is common for current experiments and other ground-based observatories. Depending on the science under investigation, subarray operation may be required. Each site may therefore be conducting several different observation programmes concurrently.

\subsubsection{Observatory operations}

The observing programme of the CTA will be driven by the best proposals from the scientific community, which will be selected in a peer-review process. Successful applicants will provide all the information required for the optimum completion of their measurements. An observing programme will be compiled by the operations centre, taking the requirements of individual projects into account. The programme will be conducted in robotic fashion with a minimum amount of professional staff on site. Proposers are not expected to participate in measurements. Quicklook analysis will enable triggers and on-the-fly modification of projects, if required. Data and calibration files will be provided to the user. Frequent modifications to the scheduled observing programme can be expected for several reasons. Openness of triggers is essential given the transitory and variable nature of many of the phenomena to be studied by CTA. CTA must adapt its schedule to changing atmospheric conditions to ensure the science programme is optimised. The flexibility to pursue several potentially very different programmes at the same time may increase the productivity of the CTA observatory. Routine calibrations and monitoring of the array and of environmental data must be scheduled as needed to ensure the required data quality.

Observatory operations covers day-to-day use of the arrays, including measurements and continuous hardware and software maintenance, proposal handling and evaluation, automated analysis and user support, as well as the long-term programme for upgrades and improvements to ensure continued competitiveness over the lifetime of the observatory. 


\subsubsection{Data dissemination}

The measurements made with CTA will be subject to on-line analysis, including event-selection and calibration for instrumental effects. The analysis of data obtained with Cherenkov telescopes differs from the procedures typical in other wavelength ranges in that extended Monte-Carlo simulations are used to determine the effects of, and calibrate for, the influence of a large range of factors on the measurements. The necessary simulations will be carried out by CTA, used in calibrating standard pipline-processed data and will also be made available to the community for use in proposal planning etc. The principal investigators of accepted proposals will be provided with the results of standard processing and access to the standard MC simulations and the analysis pipelines used in data processing. Storage of data and archiving of scientific and calibration data, programs, and MC simulations used in the processing will be organised through the distributed computing resources made available in support of the CTA EGEE Virtual Organisation.

The processing of CTA data represents a major computational challenge. It will be necessary to reduce a volume of typically 10 TBytes of raw data per observation to a few tens of MBytes of high-level data within a couple of hours. This first-level data processing will make heavily use of Grid technology by running hundreds of processes within a global pipeline. Data processing requires also the production and analysis of the MC simulations needed for calibration. The integrated services and infrastructures dedicated to the MC production, analysis and dissemination have to be taken into account in the CTA data pipeline.

All levels of data will be archived in a standardised way, to allow access and re-processing by the scientific community. Access to all levels of data and Grid infrastructures will be provided through a single access point, the "VHE gamma-ray Science Gateway".

Figure 12 shows an overview of the integrated application e-infrastructures such as EGEE-Grid, GÉANT and CTA VO.

Fig. 12 Schematic of the integrated application of e-infrastructures like EGEE-GRID, GÉANT and VO for the CTA observatory, together with the 2009 status of the CTACG (CTA Computing Grid) project (http://lappwiki01.in2p3.fr/ CTA-FR/doku.php?id=ctacomputing-grid-public). The VO-CTA Grid Operation Centre houses the EGEE services




It is foreseen that the high level analysis of CTA data can be conducted by individual scientists using the analysis software made available by CTA. This software will follow the standards used by other high-energy observatories and will be provided free of charge to the scientific community.

\subsection{CTA organisation}

The organisation of the CTA consortium will evolve over the various stages of the project. These include:

The design study phase. Definition of the layout of the arrays, specification of the telescope types, design of the telescopes and small-scale prototyping.

The prototyping and preparatory phase. Prototyping and deployment of fullscale telescopes, preparation of the construction and installation including solving technical, organisational and legal issues, site preparation.

Construction phase. Construction, deployment and commissioning of the telescopes.

Operation Phase. Operation as an open observatory, with calls for proposals and scheduling, operation and maintenance of the facility, processing of the data and provision of analysis tools.

For the design study phase, the organisation of the consortium was defined in a Memorandum of Understanding modelled on those proven by large experiments in particle and astroparticle physics. The governing body is the Consortium Board and operational decisions are taken and work is coordinated by the Spokespersons and the Executive Board. Work Package Convenors organise and drive the work on essential parts of the project. The work packages and the area they cover are:

PHYS The astrophysics and astroparticle physics that will be studied using CTA.

MC Development of simulations for optimisation of the array layout and analysis algorithms, and for performance studies.

SITE Evaluation of possible sites for CTA and infrastructure requirements.

MIR Design of telescope optics and mirror construction.

TEL Design of telescope structure and associated drive and control systems.

FPI Development of focal plane instrumentation.

ELEC Design and development of the readout electronics and trigger.

ATAC Development of atmospheric monitoring and calibration techniques and associated instrumentation.

OBS Development of observatory operation and access strategies.

DATA Studies of data handling, processing, management and data access.

QA Quality assurance and risk assessment strategies.

The CTA design study phase was organised in terms of scientific/technical topics, rather than in terms of telescope types, to ensure that, as far as 
possible, common technical solutions are employed across the array, maximising economies of scale and simplifying array operation.

For the preparatory phase, the organisation will be adapted to the needs of the project. The Project Office will be extended, and work packages for each telescope type will be established to steer prototyping and preparations for construction. External advisors will assist in guiding and reviewing the project.

A significant task for the preparatory phase will be the definition of the legal framework and governance structure of the CTA Collaboration and observatory. Different models exist, each of which has its own advantages and disadvantages. CTA could for example be realised within an existing international organisation such as CERN or ESO. CTA could also be operated by a large national laboratory which has sufficient administrative and technical infrastructure. Suitable national laboratories exist e.g. in Germany, France, or the UK, for example. On a smaller scale, H.E.S.S. and MAGIC are operated in this mode. CTA could be established as an independent legal entity under the national law of some country, following the example of IRAM. The definition of the legal structure of CTA will be determined in close interaction with ASPERA (a group of European Research Area funding agencies which coordinates astroparticle physics in Europe). One of their main tasks is the "Implementation of new European-wide procedures for large infrastructures".

Regardless of the legal implementation, CTA management will be assisted by an international scientific and technical Advisory Board, and a Resource Board, composed of representatives of the national funding organisations supporting CTA.

Close contacts between CTA and the funding agencies (via the Resource Board) during all stages of the project are vital to secure sufficient and timely funding for the construction of the facility.

\subsection{Time schedule and costs}

CTA builds largely on proven technologies and Cherenkov telescopes of sizes similar to those needed for CTA have already been built or are in the advanced stages of construction. Remaining challenges are: (a) optimisation of the cost of telescope components; (b) improvement of the reliability of telescope components, requiring extensive prototyping; (c) establishment of the formal framework for building and operating the instrument, and the selection and provision of sites; and (d) the funding of the infrastructure.

These challenges will be addressed during the Preparatory Phase (20102013) which will be supported by an FP7 grant of up to $5.2 \mathrm{M} €$ from the European Community and by grants from various national funding agencies.

After a successful Preparatory Phase, and provided the funding has been secured, construction and deployment will then take from 2013 until 2018.

A detailed evaluation of the required construction and running costs is part of the Preparatory Phase studies. Current design efforts are conducted within an envelope of investment costs for the CTA construction and site infrastructure of $100 \mathrm{M} €$ for the southern site, featuring full energy coverage, 
and $50 \mathrm{M} €$ for the more specialised northern site (all in $2005 €$ ). CTA aims to keep running costs below $10 \%$ of the total investment, in line with typical running costs for other astrophysical facilities.

Estimates for the costs of all major components of CTA are required for any optimisation of the array design. The current model makes the following assumptions:

- The investment required to construct CTA (according to European accounting schemes) is $100 \mathrm{M} €$ for CTA-South and $50 \mathrm{M} €$ for CTA-North.

- For both sites $20 \%$ of the budget is required for infrastructure and a central processing farm. Therefore, for example, telescope construction for CTASouth is anticipated to cost $80 \mathrm{M} €$.

- The construction of the telescope foundation, optical support structure, drive/safety system and camera masts will cost $450 \mathrm{k} €$ for a $12 \mathrm{~m}$ telescope and the cost scales as (dish area) $)^{1.35}$.

- Mirrors, mounts and actuators will cost $\approx 1.7 \mathrm{k} € / \mathrm{m}^{2}$.

- Camera mechanics, photo-sensor and electronics costs will be $400 € /$ pixel, including lightcones, support structures and cooling systems.

- Miscellaneous additional costs of about $20 \mathrm{k} € /$ telescope will be incurred.

This cost model will evolve as the design work on the different components of CTA progresses.

\section{Monte Carlo simulations and layout studies}

The performance of an array of imaging atmospheric Cherenkov telescopes such as CTA depends on a large number of technical and design parameters. These include the general layout of the installation, with telescope sizes and locations, telescope optics, camera field-of-view and pixel size, signal shapes and trigger logic. In searching for the optimum configuration of a Cherenkov telescope array, one finds that most of these parameters are intimately related, either technically or through constraints on the total cost. For many of these parameters there is experience from previous gamma-ray installations such as HEGRA, CAT, H.E.S.S., and MAGIC that provide reasonable starting points for the optimisation of CTA parameters. Whilst the full optimisation of CTA has not yet been completed, extensive simulation studies have been performed and demonstrate that an array of $\geq 60$ Cherenkov telescopes can achieve the key performance targets for CTA, within the cost envelope described earlier. This section gives a summary of the most important simulation studies performed so far.

\subsection{Simulation tools}

Only a modest number of candidate configurations has been simulated in full detail during the design study, but this still required the simulation of close to $10^{11}$ proton, gamma, and electron induced showers, with full treatment 
of every interaction, tracking all the particles generated in these showers through the atmosphere, simulating emission of Cherenkov light, propagating the light down to the telescopes, reflecting it on multi-faceted mirrors, entering photomultiplier tubes, generating pulses in complex trigger electronics, and having them registered in analogue-to-digital circuits. Simulations include not only Cherenkov photons but also NSB light resulting in the registration of photons at rates of $\sim 100 \mathrm{MHz}$ in a typical photo-sensor.

Since the discrimination between $\gamma$-ray and hadron showers in CTA will surpass that of the best current instruments by a significant factor, huge numbers of background showers must be simulated before conclusions on the performance of a particular configuration can be drawn. Work is underway to reduce the CPU-time requirement by preferentially selecting proton showers early in their development if they are more likely to appear $\gamma$-like. This should lead to a substantial speed improvement in future studies. Early results from Toy models, which parametrize shower detection characteristics and are many orders of magnitude faster, are encouraging, but cannot yet be seen as adequate replacements for the detailed simulation process.

The air-shower simulation results presented here are based on the CORSIKA program [73], which is widely used in the community and very well tested. Cross-checks with the KASCADE-C ${ }^{++}$air-shower code [74] have been performed as part of this study. Simulations of the instrument response have been carried out with three codes. Two packages initially developed for H.E.S.S. (sim_telarray [75] and SMASH [76]), and one for MAGIC simulations [77], were cross-checked using an initial benchmark arrays configurations.

The large volume of simulations, dominated by those of proton-induced showers needed for background estimations, has motivated the use of EGEE (Enabling Grids for E-sciencE) for the massive production of shower and detector simulations. A Virtual Organisation has been founded and a first set of CORSIKA showers has been generated on the GRID, while a specific interface for job submission and follow-up for simulations and analysis is currently under development.

The detailed simulations described here, result in data equivalent to experimental raw data (ADC counts for each time-slice for each pixel). Analysis tools are needed to reconstruct shower parameters (in particular energy and direction) and to identify $\gamma$-ray showers against the background from hadroninitiated showers (note that the additional background from electron-induced showers is important at intermediate energies despite the much lower electron flux as electron showers are extremely difficult to differentiate from those initiated by photons). The analysis methods currently used are based on experience with past and current instruments, but are being developed to make full use of the information available for CTA, in particular to exploit the large number of shower images that CTA will provide for individual events.

The analyses in this study are based on several independent codes, all of which start with cleaning of images to identify signal pixels, and a parametrisation of images by second-moment Hillas parameters [78], augmented by parameters such as the height of shower maximum as reconstructed from stereo 
images. Background rejection is achieved both by direct cuts on (suitably normalised) image parameters, and more general multivariate analysis tools such as a Random Forest [79] classifier and Boosted Decision Trees within the open source software package TMVA (http://tmva.sourceforge.net) [80, 81]. There are also other analysis methods in use for the analysis of Cherenkov telescope data, such as the 3-D-model analysis [82] the Model++ analysis [68], and analytical combinations of probability density functions of discriminating variables which have advantages over the standard second-moments analysis in at least some energy ranges. Some of these alternative methods have been used for a subset of the studies presented here.

\subsection{Verification of simulation tools}

The optimisation of CTA relies heavily on detailed simulations to predict signal and background rates, angular resolution and overall sensitivity. To demonstrate that the simulation tools in use accurately describe reality, we show here some key data/simulation comparisons, taking H.E.S.S. as an example.

A key aspect of the simulation of the detector response to Cherenkov light from an air-shower is the ray-tracing of light through the optical system of an individual telescope. An understanding of the typical misalignments of all components is needed at this stage, as is the ideal performance. The optical performance of a telescope is described by its point spread function (PSF), which degrades for off-axis rays. Figure 13 illustrates that the modelling of the optical system of, in this case, a H.E.S.S. telescope reproduces the width and

Fig. 13 Optical point spread function of two H.E.S.S. telescopes as a function of angle of incidence, measured using stars, and compared to simulations. Data points are shown for the radial and tangential width of the PSF, and the $80 \%$ containment radius. Lines represent the results of simulations of the telescope optics using sim_telarray. See [83] for details

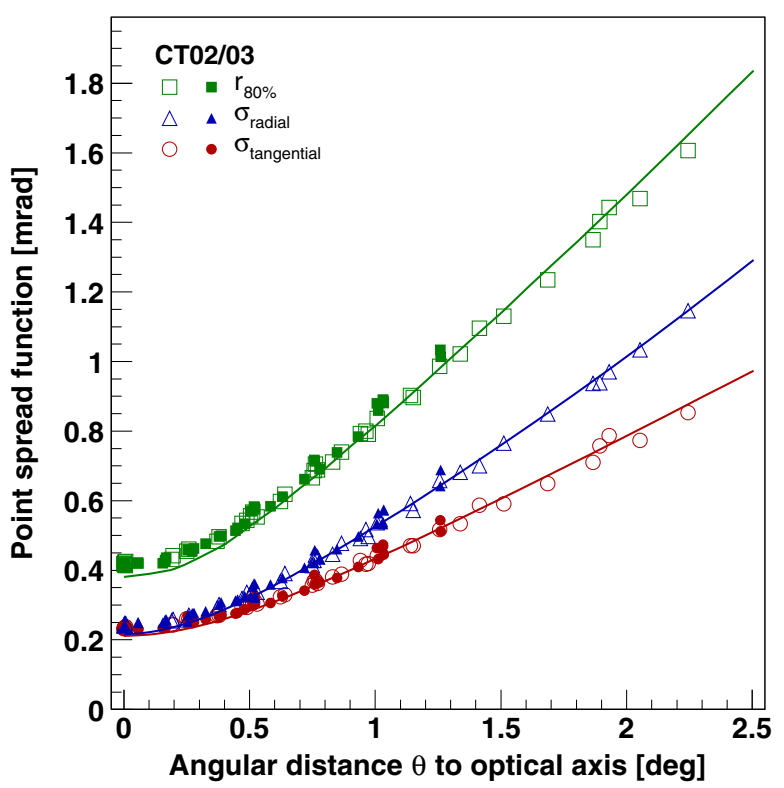


Fig. 14 Comparison of measured (black squares) and simulated (red triangles and blue circles) image parameters for the H.E.S.S. telescopes. Measured data are taken from a flare of the blazar PKS 2155-304 [84] for which the signal/noise ratio was very high and large gamma-ray statistics are available

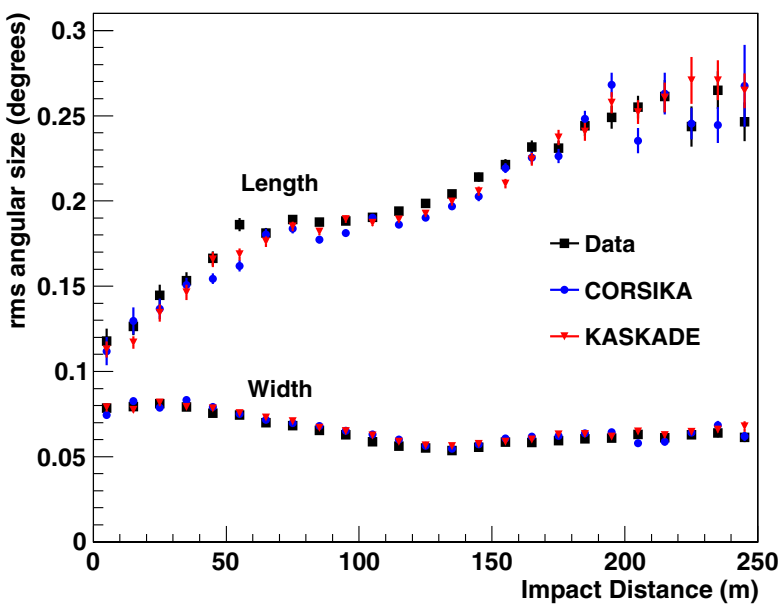

shape of the PSF in all details, and that essentially identical imaging is achieved for different telescopes in the system.

An end-to-end test of the correct simulation of gamma-ray induced showers can be made using the signal from a strong source under very high signal/background conditions. The giant flare from the blazar PKS 2155-304 observed with H.E.S.S. in 2006 provides an excellent opportunity for such a test. Figure 14 shows the satisfactory agreement (typically at the 5\% level) between the simulated and detected shape of the shower image as characterised by their Hillas width and length parameters. Gamma-ray showers were simulated with the CORSIKA and KASKADE-C ${ }^{++}$programs and have been passed through one of the H.E.S.S. detector simulation and analysis chains. The measured spectrum, optical efficiency, zenith angle and other runtime parameters were used as inputs to this simulation.

In the analysis of experimental data, it is sufficient for simulations to describe the characteristics of gamma-ray detection, since the cosmic-ray background can (except for very diffuse sources) be modelled and subtracted using measurements in regions without gamma-ray emission. However, for the design of new instruments, simulations must also provide a reliable modelling of all relevant backgrounds. Experience with existing systems shows that this is indeed possible, provided that background events are simulated over a very wide area, up to an impact distance of around a kilometre from any telescope and over a large solid angle, well beyond the direct field of view of the instrument, so that far off-axis shower particles are properly included.

An inherent uncertainty in the simulation of the hadronic background is given by the currently limited knowledge of hadronic interaction processes at very high energies. The impact of this uncertainty on the Cherenkov light profile has been studied using CORSIKA simulations with different interaction models. As can be seen in Fig. 15, the low energy $(<80 \mathrm{GeV})$ models FLUKA [85] and UrQMD [86] do not exhibit significant differences, whereas the known discrepancy between the high-energy models QGSJet- 

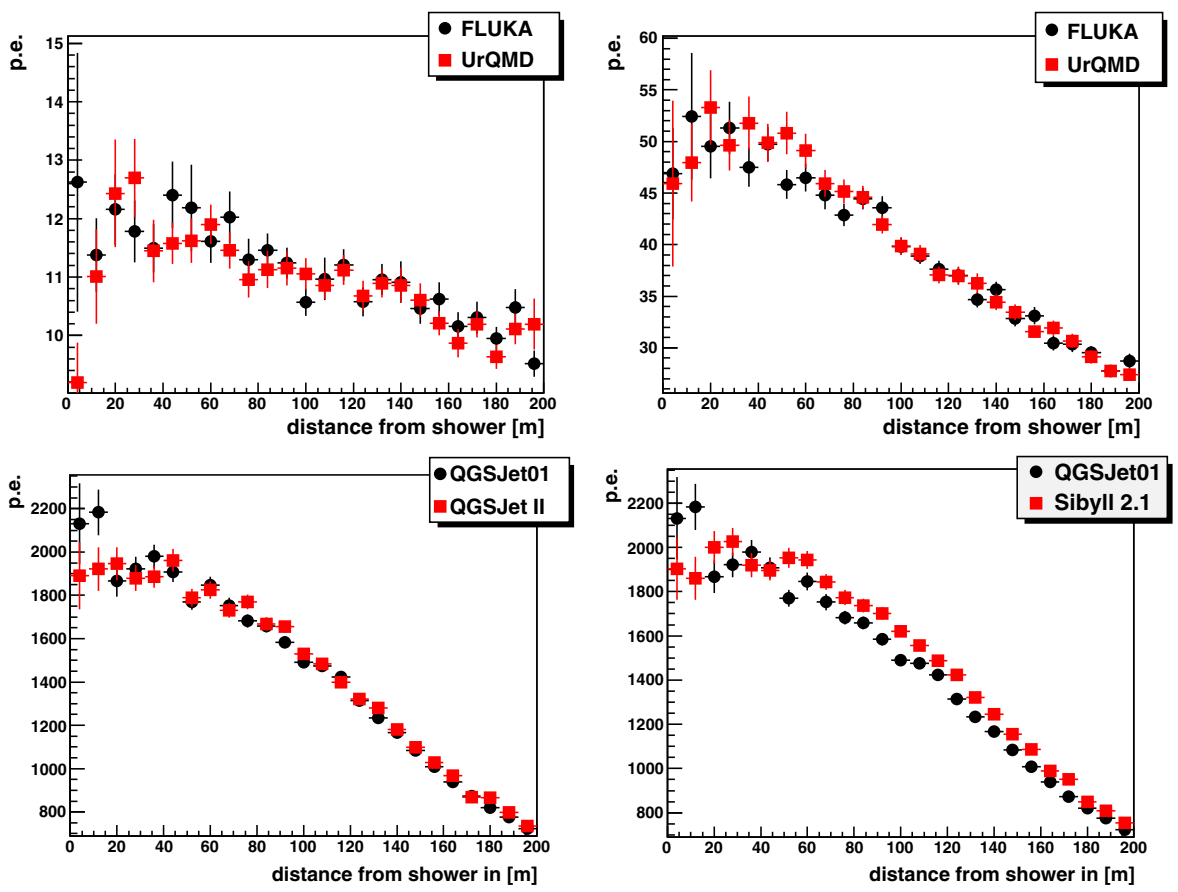

Fig. 15 Comparison of the Cherenkov light profiles for proton-induced showers generated with different hadronic interaction models. The profiles for FLUKA and UrQMD at $50 \mathrm{GeV}$ (left) and $100 \mathrm{GeV}$ (right) are shown in the top panel. Two QGSJet versions and SIBYLL at $1 \mathrm{TeV}$ are compared in the bottom panels

01 [87], QGSJet-II [88, 89] and SIBYLL 2.1 [90] leads to an uncertainty of about $5 \%$ in the Cherenkov light profile at $1 \mathrm{TeV}$.

As can be seen in Fig. 16, the raw cosmic-ray detection rate as a function of zenith angle is described to within about 20\%. Given the uncertainties on cosmic-ray flux, composition above the atmosphere and in the hadronic interaction models, better agreement cannot be expected. In the backgroundlimited regime this uncertainty corresponds to a $10 \%$ uncertainty in sensitivity, assuming that the fraction of $\gamma$-like events is understood. Figure 17 demonstrates that the fraction of such events, and the distributions of separation parameters, are indeed well understood for instruments such as H.E.S.S. using the simulation and analysis tools applied here to CTA.

\subsection{Energy range and sensitivity of telescope arrays}

Three methods of representing the sensitivity of a Cherenkov telescope are used in the following discussion. All three have merits and emphasise different features. The traditional way to represent the sensitivity of Cherenkov Telescope systems is in terms of integral sensitivity, including all events reconstructed above a given energy (and often multiplied by the threshold energy 
Fig. 16 Dependence of H.E.S.S. system trigger rate on zenith angle, for data and simulations. The simulations assume two different model atmospheres, with the atmosphere at the H.E.S.S. site representing an intermediate case. See [72] for more details

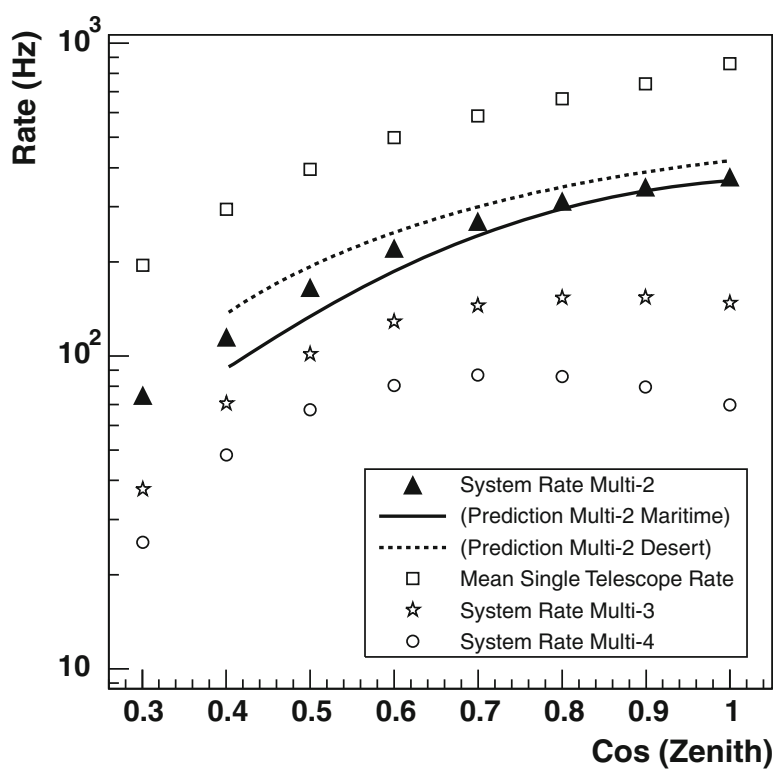

to flatten the curves and give more useful units of $\mathrm{erg} /\left(\mathrm{cm}^{2} \mathrm{~s}\right)$. An observation time of 50 hours (typical for the first generation of IACTs) is assumed for comparison to published sensitivity curves of historical and current instruments. Integral sensitivities depend on the assumed source spectrum and can be deceptive in that much of the detection power quoted for a given threshold may actually be derived from events well above that threshold. A

Fig. 17 Measured distribution of the proton/electron separation parameter $\zeta$ for 239 hours of H.E.S.S. data on sky fields without gamma emission, compared to simulations of proton- and electron-induced showers. The shape of the background is very well reproduced by simulations across the full range of $\zeta$. Gamma-ray signals appear close to $\zeta=1$. The electron background is therefore important despite the relatively low flux of electrons in comparison to hadrons. See [91] for more details

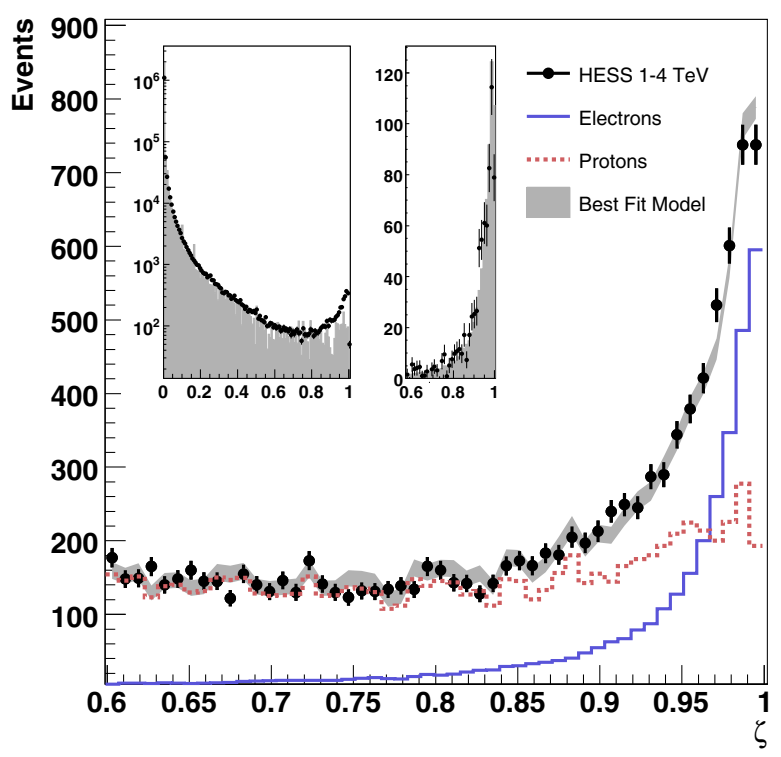


more useful, but less common, way to represent the sensitivity of IACTs is in terms of differential sensitivity, where a significant detection (above $5 \%$ of the background level, with $\geq 5 \sigma$ statistical significance and at least ten events) is required in each energy bin. Five bins per decade in energy are used for the following results for possible CTA configurations. The differential flux sensitivity is sometimes multiplied by $E^{2}$ to show the minimum source flux in terms of power per logarithmic frequency interval and given in units of erg $\mathrm{cm}^{-2} \mathrm{~s}^{-1}$ for ease of comparison with other wavebands. Alternatively, the Crab nebula, as a strong and non-variable gamma-ray source with a rather typical spectral shape, can be used as a reference. Here we use the VHE spectrum as measured with the HEGRA telescope array as a reference, i.e. 1 Crab Unit $(\mathrm{CU})=2.79 \times 10^{-11} E^{-2.57} \mathrm{~cm}^{-2} \mathrm{~s}^{-1} \mathrm{TeV}^{-1}$. (Note that the true spectrum of the Crab nebula falls below this expression at the highest and lowest energies.)

Several different telescope configurations have been investigated in simulation studies for CTA so far. The first simulations were used to cross-check the different simulation packages and to begin the investigation of the dependence of performance on telescope and array parameters. Selected results from one of these, an array of nine telescopes with $24 \mathrm{~m}$ diameter (the "benchmark" array), are discussed below. Following these studies a series of simulations were conducted with larger telescope arrays (including $41 \times 12 \mathrm{~m}$ telescopes and a 97-telescope array with two different telescope sizes) to demonstrate that the goals of CTA are attainable with a large telescope array (see [92]). More recently, a 275 telescope "production configuration" has been simulated, subsets of which constitute CTA candidate configurations. So far 11 candidate configurations have been defined with an approximately equal construction cost of about $80 \mathrm{M} €$ (in $2005 €$ ) with the current CTA cost model.

The evaluation of the performance of these candidate arrays is a first step towards the optimisation of the CTA design. Figure 18 shows some of the telescope layouts used. All systems assume conventional technology for mirrors, PMTs and read-out electronics. Standard analysis techniques are used in general, with the results from more sophisticated methods shown for comparison in specific cases.

The nine-telescope benchmark array has been used to test several aspects of array performance, in particular the desirable altitude range and best pixel size for the lower part of the CTA energy range. Figure 19 compares arrays located at different elevations (2,000, 3,500 and 5,000 m) and also illustrates the influence of systematic errors in the background determination at low energies. The spacing of telescopes is adjusted to compensate for the changing radius of the Cherenkov light-pool with altitude. For 2,000 $\mathrm{m}$ elevation, the array has useful sensitivity above $\approx 20 \mathrm{GeV}$ and at higher energies dips below the $1 \% \mathrm{Crab}$ level. An equivalent system at high elevation $(5,000 \mathrm{~m})$ provides a lower threshold but worse performance at high energies, at least partly reflecting the smaller diameter of the light pool at high altitude and hence the reduced detection area. Another potential problem at very high altitudes is the contamination of the signal by Cherenkov light from individual shower particles which reach the observation level. Sensitivities cross at about $30 \mathrm{GeV}$, 

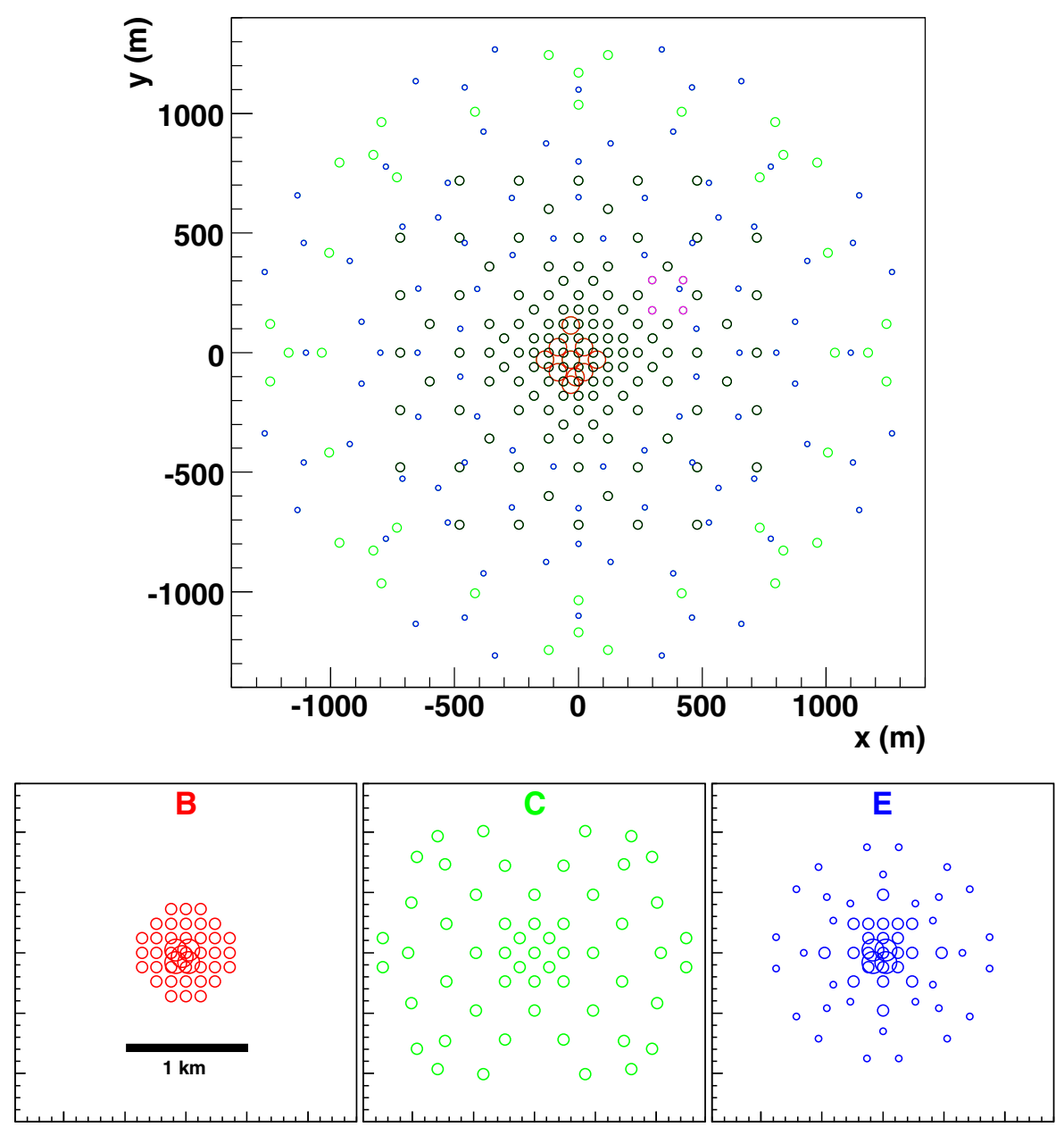

Fig. 18 Top 275 telescope super-configuration for the MC mass production. Five telescope types are simulated (red: $24 \mathrm{~m}$ diameter telescopes, black and green: $12 \mathrm{~m}$, pink: $10 \mathrm{~m}$, blue: $7 \mathrm{~m}$ ), with the circle size proportional to the mirror area. Bottom Three example candidate configurations (B, C and E) which are subsets of the 275 telescope array and would all have an approximate construction cost of $80 \mathrm{M} €$

implying that a high-altitude installation is mainly relevant for specialised very-low-energy instruments, such as the 5@5 array [93]. Similar conclusions were reached in earlier simulations by Plyasheshnikov (private communication) and Konopelko [94]. A 3,500 m altitude array delivers a somewhat lower energy threshold than one at $2000 \mathrm{~m}$ and comparable performance at $0.1-1 \mathrm{TeV}$ for the benchmark array. However, it is not clear that this result on relative performance at intermediate energies can be generalised to the much larger telescope array of smaller telescopes with which CTA plans to cover this energy range. Simulations of the 275-telescope array at 3,700 m altitude are underway to address this question. 
Fig. 19 Differential sensitivity (with five independent bins per decade in energy) of the nine-telescope benchmark array placed at 2,000, 3,500 and at 5,000 m elevation, for point sources observed for 50 $\mathrm{h}$ at a zenith angle of $20^{\circ}$. A $5 \sigma$ significance, at least ten signal events, and a signal exceeding $5 \%$ of the remaining background is required for a detection. The image cleaning method applied uses dual threshold $5 / 10$ photoelectron



Figure 20 shows the impact of changing the (angular) pixel diameter $\left(\Theta_{p}\right)$ on the sensitivity of the benchmark array at 2,000 $\mathrm{m}$ altitude. It can be seen that only modest improvements are possible with pixels below $0.1^{\circ}$ diameter. As the camera cost increases as $1 / \Theta_{p}^{2}$, smaller pixels sizes are strongly disfavoured. The improvement of angular resolution at smaller pixel size is also found to be modest in our studies (see also [95]). Alternative analyses may lead to significant benefits from smaller pixel sizes, but this has not yet been demonstrated.

The 275-telescope production configuration described above is the focus of the current work within CTA and has been used to demonstrate the validity of the CTA concept. Figure 21 shows some example events as seen in a candidate sub-configuration of this production array, demonstrating the high telescope multiplicity (and event quality) which is a key element of the CTA design.

Figure 22 shows how the angular resolution defined as the $68 \%$ containment radius, improves with the number of telescopes that record a shower image. With four images (as for instruments like H.E.S.S. or VERITAS) a

Fig. 20 Differential sensitivity curves for the nine-telescope benchmark array for several different pixel sizes using the same criteria as for the previous figure. Image cleaning is adapted to the respective noise levels in each case. The impact of reduced pixel size is mainly visible close to the threshold energy





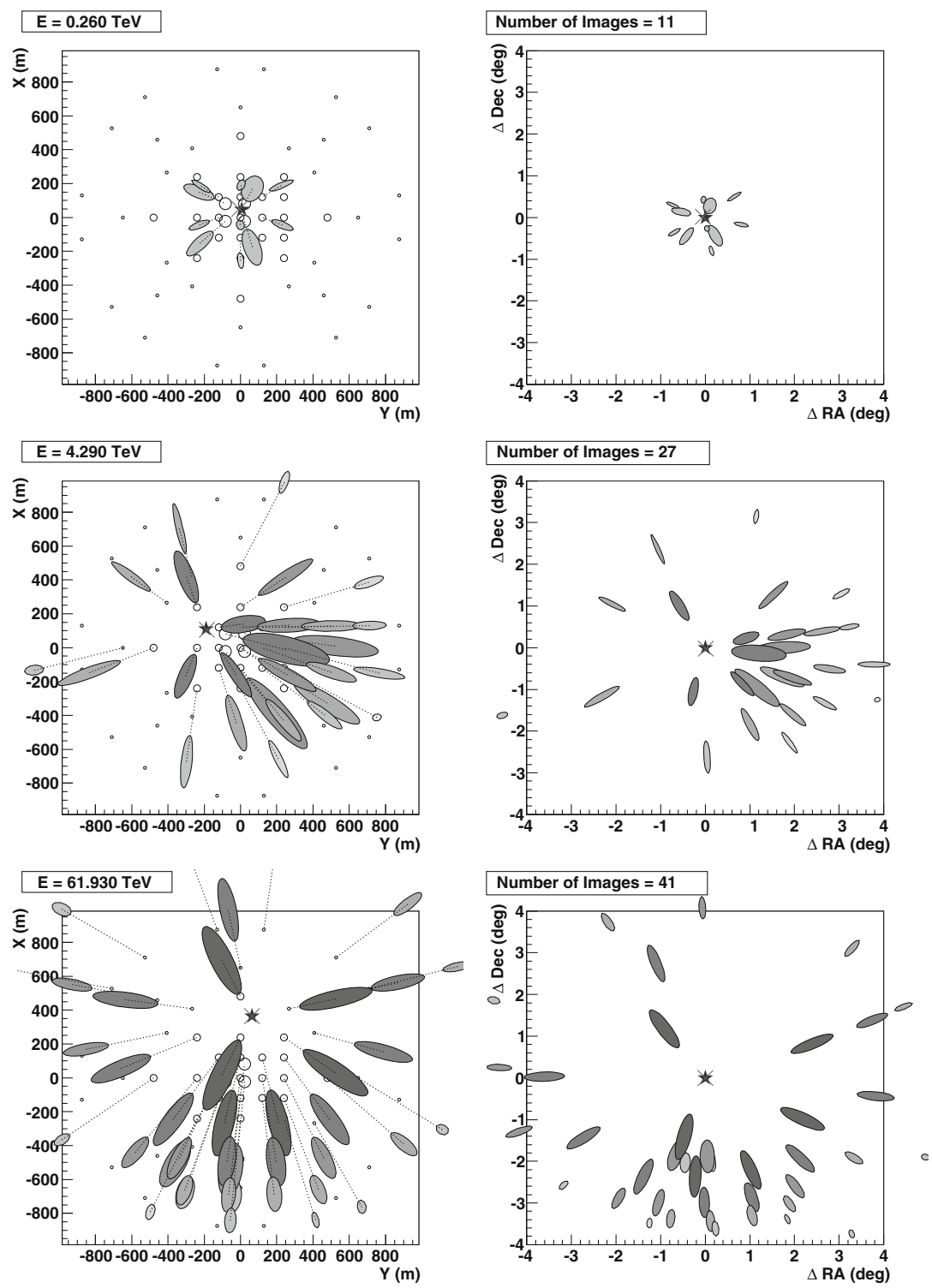

Fig. 21 Three events as seen by the 59-telescope candidate array E. The gamma-ray energy and number of images seen are shown in each instance. The left-hand plots show the telescopes on the ground (the three sizes of circles for the telescopes of diameters 7,12 and $24 \mathrm{~m}$, respectively), with projected Hillas ellipses drawn relative to each telescope position for each triggered telescope. Higher amplitude images are filled with darker grey. The point of intersection of the primary trajectory with the ground is marked with a star. It is found in a simultaneous fit of both core and direction. The truncation of images at large impact distances is clearly visible. The right-hand plots shows the same ellipses in the camera plane, with the gamma-ray source position marked with a star. (In the most rudimentary analysis one can reconstruct the impact point on ground by the intersection of the directions from image centroids to each of their telescope positions (dotted lines on the left), and the gamma-ray direction in the sky from the intersection of the image axes $($ right $))$ 
Fig. 22 Angular resolution (68\% containment radius) for array configuration $E$, as a function of the number of telescopes with good shower images

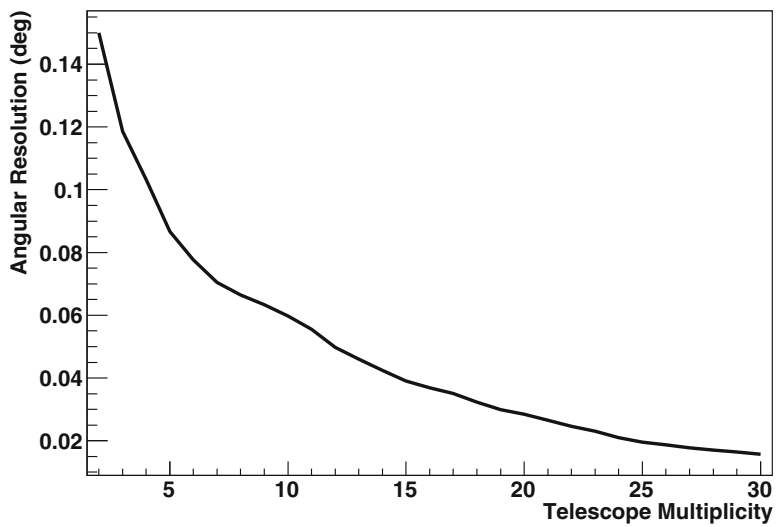

resolution of about $0.1^{\circ}$ is reached, while with $\geq 12$ images the resolution is $\leq 0.05^{\circ}$. For the most energetic showers, resolutions of $<0.02^{\circ}$ are reached. Analogous simulations for AGIS [96] give a very similar angular resolution. The telescopes simulated include one type of $12 \mathrm{~m}$ diameter, $8^{\circ}$ field-of-view and $0.18^{\circ}$ pixels (squares in Fig. 21, used in configurations B, C and E), one type of $7 \mathrm{~m}$ diameter, $10^{\circ}$ field-of-view and $0.25^{\circ}$ pixels (triangles in Fig. 21, used in configuration $\mathrm{E}$ ) and a 24-m telescope type with $5^{\circ}$ field-of-view and $0.09^{\circ}$ pixels (circles in Fig. 21, used in configurations B and E). The 24-m telescopes use parabolic optics, all other telescopes are based on the Davies-Cotton design. Optical designs intermediate between parabolic and Davies-Cotton are now under consideration to optimise the trade-off between time-dispersion and off-axis performance. For the cameras, a quantum efficiency curve of similar spectral shape (blue-sensitive) to that of current bi-alkali PMTs is assumed. This is a conservative assumption as $\sim 50 \%$ higher efficiency cathodes have recently been announced by several major manufacturers (albeit with larger after-pulsing rates, which may limit the advantage gained in terms of trigger threshold).

Figure 23 illustrates the integral flux sensitivity achieved with the three candidate CTA configurations shown above. The goal sensitivity curve for CTA is shown for comparison. It can be seen that these configurations (even with rather basic analysis methods) are close to achieving the goal performance in most energy ranges. At very high energies it seems to be possible to exceed the original goal performance by a significant factor within the nominal project budget. As the three configurations $\mathrm{B}, \mathrm{C}$ and $\mathrm{E}$ have roughly equal cost, they can be used to show the impact of changing the energy emphasis of the observatory on the performance achieved. Configuration $\mathrm{C}$ covers a very large area $\left(\sim 5 \mathrm{~km}^{2}\right)$ but lacks any telescopes larger than $12 \mathrm{~m}$ and hence has very little sensitivity below $100 \mathrm{GeV}$. Configuration B has a low-energy core of 24-m telescopes surrounded by a closely spaced 12-m telescope array. This configuration provides superior hadron rejection and angular resolution (see later) but provides a more modest effective collection area at multi-TeV energies. Configuration $\mathrm{E}$ is a compromise array, which attempts to do well in 
Fig. 23 Integral sensitivity (multiplied by $E$ ) for the candidate configurations B, $\mathrm{C}$ and $\mathrm{E}$, for point sources observed for $50 \mathrm{~h}$ at a zenith angle of $20^{\circ}$. The goal curve for CTA (dashed line) is shown for comparison

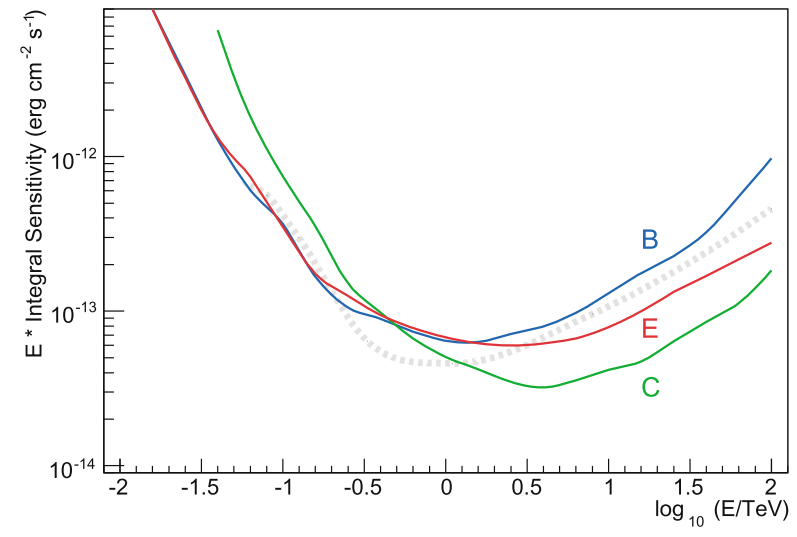

all energy ranges using multiple telescope types and spacings. As can be seen from Fig. 23, such an array comes closest to achieving the CTA performance goals.

It is important to study the potential sensitivity of CTA at much shorter observation times than the $50 \mathrm{~h}$ used for reference. Figure 24 shows how the sensitivity changes for 5 -h and 0.5 -h observations. The sensitivity scales linearly with time $t$ in the regime limited by gamma-ray statistics and approximately with $\sqrt{t}$ in the background limited regime at lower energies. For candidate array E, the detection of a source with $2 \%$ of the Crab Nebula flux (the flux level of the weakest known sources of VHE gamma-rays until 2007) would be possible in just over $30 \mathrm{~min}$. Extreme AGN outbursts, which in the past have reached flux levels $>10 \times$ the Crab flux, could be studied with a time resolution



Fig. 24 Time and energy dependence of the differential sensitivity (for five independent measurements per decade in energy, multiplied by $E^{2}$ ) for configuration E. Exposure times of 0.5, 5 and $50 \mathrm{~h}$ are shown. Selection cuts were optimised separately for each exposure time. For the 50 -h curve two alternative analysis methods are also shown. The red curve is for an analysis procedure with an image cleaning procedure and a Random Forest-based method for hadron rejection. An independent analysis using TMVA for hadron rejection is shown as a blue curve 
Fig. 25 Angular resolution (68\% containment radius of the gamma-ray PSF) versus energy for the candidate configurations $\mathrm{B}, \mathrm{C}$ and $\mathrm{E}$. The resolution for a more sophisticated shower axis reconstruction method for configuration $\mathrm{E}$ is shown for comparison (dashed red line- $\left.\mathrm{E}^{*}\right)$. The angular resolution of H.E.S.S. (basic Hillas analysis, standard cuts) is shown as a reference [97]

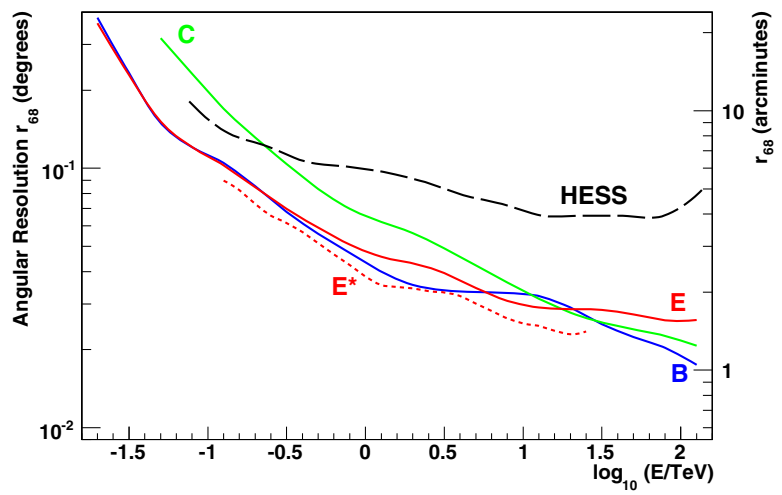

of seconds, under virtually background-free conditions. Figure 24 also shows 50-h sensitivity curves calculated using two independent analyses, illustrating (a) that the conclusions on sensitivity presented here are robust and (b) that the sensitivity can be improved using more advanced methods for background suppression over much of the CTA energy range.

The angular resolution for the CTA candidate systems is summarised in Fig. 25. Resolution at $1 \mathrm{TeV}$ is in the $0.04-0.05^{\circ}$ range for configurations $\mathrm{B}$ and $\mathrm{E}$, and somewhat worse for the larger area configuration $\mathrm{C}$, illustrating the trade-off between collection area and precision at fixed cost. A simultaneous minimisation to find the best shower core and direction, using pixel timing information, provides a significant improvement over the traditional intersection of image axes technique (see dashed line in Fig. 25). The resolution approaches $1 \mathrm{~min}^{-1}$ at high energies. Fiducial cuts on core location and/or harder telescope multiplicity cuts improve this performance, at the expense of collection area.

The energy resolution (for photon showers) as a function of energy is shown in Fig. 26 for the candidate arrays B, C and E. The energy resolution is below $30 \%$ in almost the whole range of interest and $\leq 10 \%$ above about $1 \mathrm{TeV}$.

Fig. 26 Energy resolution versus energy for the candidate configurations $\mathrm{B}, \mathrm{C}$ and $\mathrm{E}$

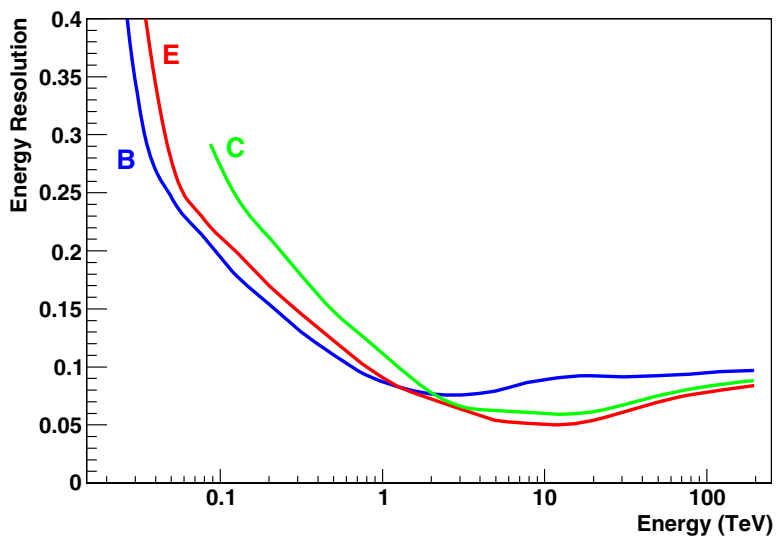


In summary, whilst the final optimisation of the CTA design will require accurate cost models and input from quantitative "key science projects", it is clear from our current studies that an array of $\sim 60$ wide field of view Cherenkov telescopes can achieve the key performance goals of CTA within the envisaged level of investment.

\section{CTA telescope technology}

A particular size of Cherenkov telescope is only optimal for covering about 1.5-2 decades in energy. Three sizes of telescope are therefore needed to cover the large energy range CTA proposes to study (from a few tens of $\mathrm{GeV}$ to above $100 \mathrm{TeV}$ ). The current baseline design consists of three single-mirror telescopes: SST: Small size telescopes of 5-8 m diameter; MST: Medium size telescopes of 10-12 m diameter; and LST: Large size telescopes of 20-30 m diameter.

While telescope optics involving multiple reflectors or optical correctors have been proposed $[66,98,99]$ and do provide improved and more uniform imaging across large fields of view, these designs are also more complicated than the classical single-reflector Cherenkov telescopes. Single-reflector designs are adequate for the fields of view necessary for CTA and provide a PSF well-matched to the proposed PMT-based camera. Imaging is improved by choosing relatively large $f / d$ values, in the range of 1.2-1.5. A second variable is the dish shape: a Davies-Cotton layout provides good imaging over wide fields, but introduce a time dispersion. For small dish diameters this dispersion is smaller than the intrinsic width of the photon distribution, and therefore insignificant. For large dish diameters, the difference in photon path length from different parts of the reflector becomes larger than the intrinsic spread of photon arrival times, broadening the light pulse. A parabolic shape, which does not introduce this dispersion, is therefore preferred for very large telescopes. The transition between the two regimes is at about the size of the MST. Other alternative dish shapes face the same general trade-off between time dispersion and imaging quality.

\subsection{Telescope mount and dish}

One of the most important mechanical components of a telescope is the mount, with its associated drive systems. This must allow the slewing of the dish and the tracking of celestial objects. The dish structure supports the segmented reflector and the camera support which holds the camera at the focus on the reflector. Critical properties for the structural components of a telescope include:

Positioning of mirror facets. The dish structure supports mirror facets forming a parabolic or Davies-Cotton reflector. Its prime task is to keep the relative orientation of the mirror facets stable at the arcminute level. 
Mechanical stability of the optical system. Stability must be achieved under observing and "survival" conditions. Typical camera pixel sizes are 5-10'. To achieve a stable focus, independent of pointing, modest wind loads and temperature variations, mirror facets have to be kept stable to well below $1^{\prime}$, either by a suitably stiff structure and/or by active mirror attitude control. Survival conditions refer to high wind and snow loads, which the telescope must tolerate without suffering damage.

Pointing and tracking precision. The effective optical pointing of a telescope, i.e. the location of images on the camera, is determined by the precision of the tracking system, the overall deformations of the dish and the deformations of the camera support. Given the extremely short exposure times (ns), the pointing does not need to be stable or precise to more than a few arcminutes, provided that the effective pointing is monitored with sufficient precision.

Slewing speed. A slewing speed that allows repointing to any location in the sky within a minute is normally sufficient, given that objects are usually tracked over tens of minutes before repositioning. Only for one special class of targets, the GRB alert follow-ups, is the fastest possible slewing desirable. Faster slewing of $180^{\circ}$ in $20 \mathrm{~s}$ is planned for the large-sized telescopes, which are most suited for such follow-ups, given their low energy threshold.

Efficiency of construction, transport, and installation. This is a key factor in reducing costs. For mass production of telescopes, it may be most efficient to set up a factory for assembly of structural components at the instrument site, avoiding shipment of large parts and minimising tooling.

Minimal maintenance requirements. Reducing on-site maintenance to a minimum aids high efficiency operation and minimises the requirements for on-site technical staff.

Safety considerations. All procedures for installation and maintenance have to ensure a high level of safety for workers. The telescopes must also be constructed so that even in the case of failures of the drive systems or power they can be returned to their parking positions.

\subsubsection{Mounting system and drives}

While some of the very first Cherenkov telescopes were equipped with equatorial mounts, alt-azimuth mounts offer obvious advantages and have been adopted for all modern instruments. Two main types of mounts are in use (Fig. 27):

Circular rail system for azimuthal motion, supporting the dish between two elevation towers, as is used by H.E.S.S. and MAGIC. The elevation axis is positioned such that the dish is balanced and little or no counterweight is required. This support scheme will in general permit a large movement range in elevation, allowing the positioning of the camera near ground level for easy access, and the tracking of sources which go through the 

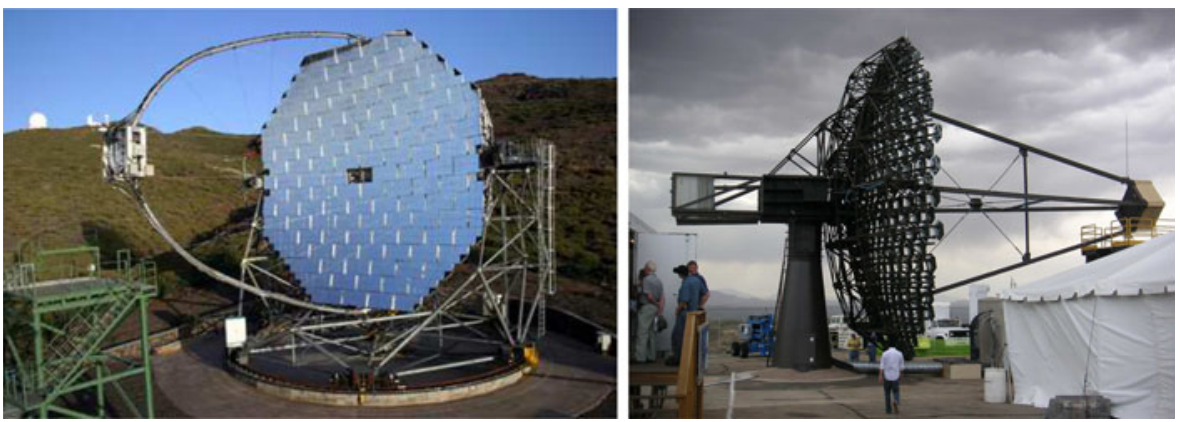

Fig. 27 Examples of alt-azimuth mount, as used for H.E.S.S. and MAGIC (left) and a central positioner design, used for the Whipple and VERITAS telescopes (right)

zenith without repositioning by $180^{\circ}$ in azimuth. A disadvantage of a rail system is the considerable on-site effort required: a large ring foundation must be constructed, the azimuth rail needs to be carefully levelled, and drive systems have to be mounted and cabled on-site.

The central positioner as used by VERITAS, in which the dish is supported from near its center in the back. The central positioner construction is often used for radio and radar antennae and mirrors for solar power concentrators. The construction of the foundation is considerably simplified and the on-site installation work reduced which can be of importance at sites with poor access or difficult terrain. In addition, maintenance tends to be simplified since all bearings and drive components are contained and protected within a compact positioner unit, as opposed to rails and wheels which are more exposed. While these advantages make the choice obvious for antennae and solar concentrators, for which focal plane instrumentation is generally of low weight and $f / d$ is normally very short, the trend for Cherenkov telescopes is now towards large $f / d$ ratios, well above 1 , to provide improved image quality. More and more components are also being installed in the camera, resulting in increased weight. Large counterweights are then required to balance the elevation axis in the central positioner design, as is visible in the VERITAS case. Without these counterweights, the elevation mechanism has to handle large torques and the desired positioning speeds require much larger drive power than needed for balanced systems. Access to the camera at ground level is also possible in these designs if one locates the elevation axis away from the centre of the tower.

Alternative mounting schemes have been considered. For example, a hexpod mount was investigated for the H.E.S.S. II telescope, but was abandoned as the initially assumed cost advantages over conventional mounts turned out to be marginal due to the complexity of the hydraulic drive system and the extensive safety features required. In addition, a hexpod mount requires a 
mirror cover during day time, when the dish is parked facing up. Camera access is also non-trivial.

Another unconventional mounting scheme is a lift-up mirror carried on a circular rail, which eliminates the elevation towers and, at least in some dish support schemes, allows the reduction of bending torques on the dish due to the camera support system. A conceptual design for such a scheme was worked out for H.E.S.S. (see Fig. 28, left), but again did not offer cost advantages. With a different elevation mechanism, this support scheme has been considered for the medium-sized CTA telescope (Fig. 28, right). A drawback of such systems is that the centre of gravity moves as the telescope's elevation is changed, requiring significantly increased drive power compared to balanced systems, where the drives only have to counteract friction, inertia, and certain wind loads.

For the LST, only a rail design, as used by H.E.S.S. and MAGIC, appears feasible. This is also a possible solution for the MST, although here a central positioner is a viable option. The solution chosen for the mount has significant influence on the dish design. When a rail mount is used, the dish is supported either at its circumference, requiring a stiff dish envelope, or via an extra elevation cradle as used in the H.E.S.S. II telescope. With a central positioner, the dish is supported from its centre, and loads at the periphery of the dish must be minimised. For the SST with its reduced weight and loads, it appears cost effective to use a central positioner type mount as illustrated in Fig. 29(left) or to support the telescope by elevation towers but replace the rail by a central azimuth bearing as is used in the HEGRA telescopes (Fig. 29, right).
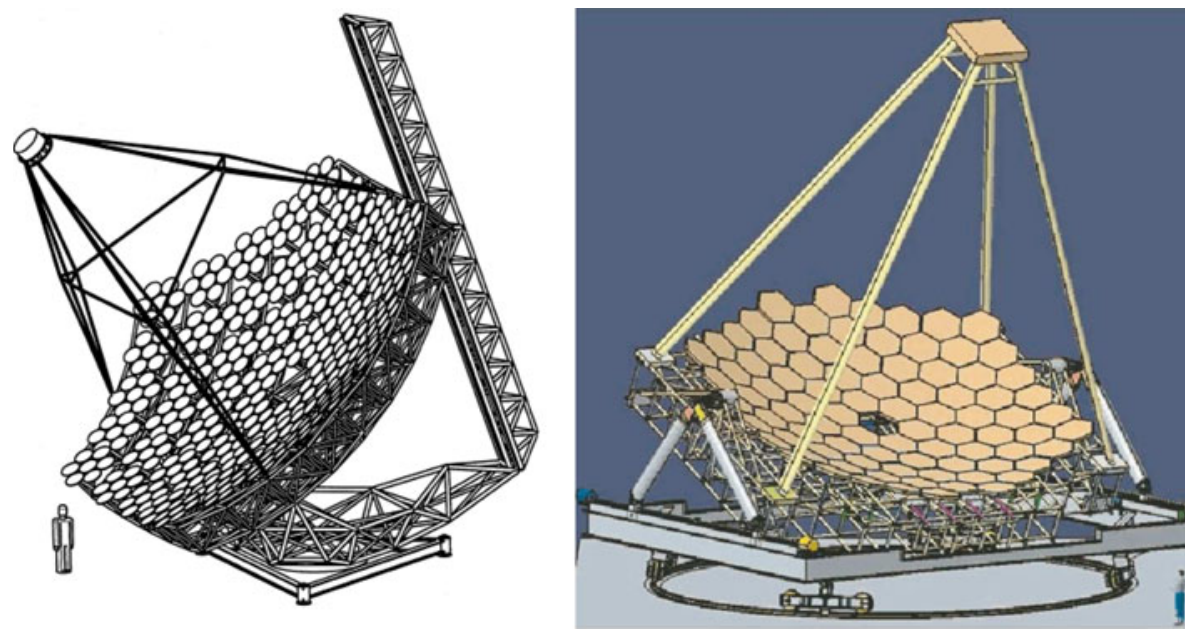

Fig. 28 Alternative alt-azimuth mounts, eliminating the elevation towers, as studied for H.E.S.S. (left) and CTA (right) 

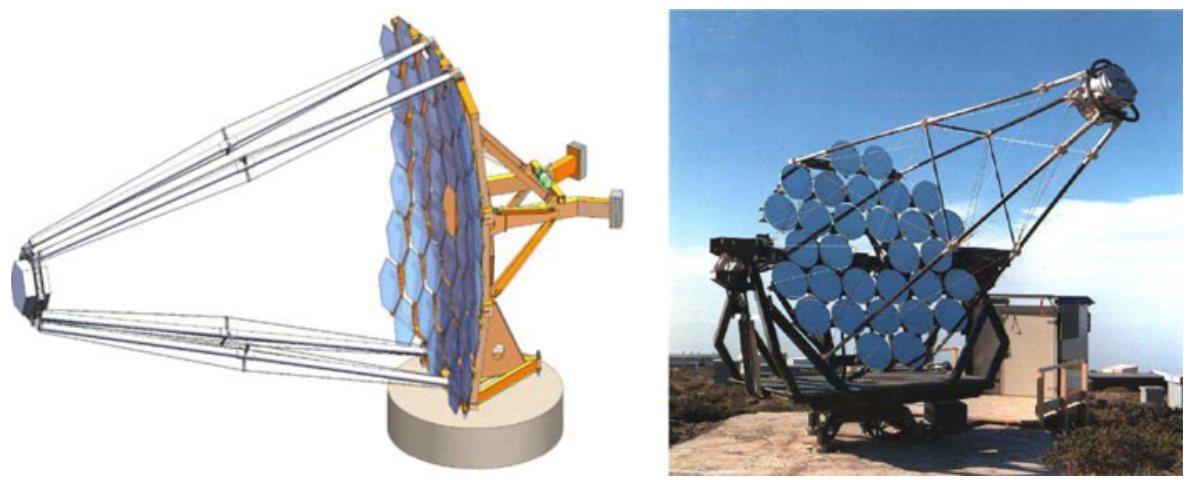

Fig. 29 Two options for the SST mount: a central positioner (left) or a HEGRA-type support (right)

Various types of drive systems are implemented in current telescopes. The experience gained with these will inform the CTA designs. Some central positioners can be purchased as commercial units and others are under development with industrial partners. The main challenge is the large torque that must be transmitted by a rather compact unit, resulting in high forces on gears and bearings. Dual counter-acting drive units are unavoidable to compensate for play. For rail-based mounts, azimuth drive systems are used, e.g. friction drives (H.E.S.S. I), multiple driven wheels (H.E.S.S. II) and rack-and-pinion drives, implemented using a chain (MAGIC). For the elevation drive of the LST, a rack-and-pinion system is being considered, again with the option of using a chain. For the SST and the MST, directly driven elevation axes are an option.

Commercial servo systems will be used to control the drive motors, with multiple feedback loops: for example, H.E.S.S. II e.g., uses an inner feedback loop to control motor speed and/or torque, implemented in the servo controller, an intermediate fast software-based feedback loop implemented in a local controller to control axis motion and to balance multiple drive motors acting on an axis, and an outer slower software-based feedback loop for absolute positioning and tracking, based on absolute shaft encoders. Relatively low-cost encoders provide a precision of $\leq 10^{\prime \prime}$. At this level, pointing precision is usually dominated by deformations of the dish and of the camera support, causing deviations of the effective optical axis from the nominal pointing monitored by the encoders.

Pointing can be corrected by a combination of lookup-based corrections of elastic deformations, star guider CCD cameras monitoring the actual orientation of the dish, and CCD cameras monitoring the position and orientation of the focal plane instrumentation relative to the dish axis. Using a combination of such measures allows an (off-line) pointing accuracy of about $10^{\prime \prime}$ to be achieved. 

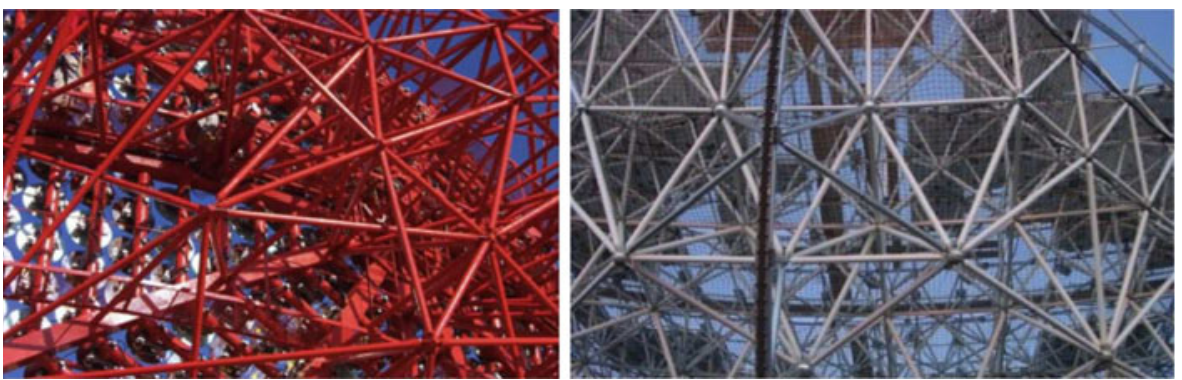

Fig. 30 Examples of the space-frame construction. H.E.S.S. steel space-frame (left) and the MAGIC three-layers CFRP space-frame (right)

\subsubsection{Dish structure and camera support}

The dish structures of the LST that is currently planned, has a space frame similar to that used in different variants in the H.E.S.S., MAGIC and VERITAS telescopes (Fig. 30). A designs with only a minimal space frame is favoured for the dish of the MST. Another option is a relatively coarse space frame with an additional structure to provide mirror attachment points. Alternatively, one can use a highly resolved space frame, based e.g. on tetrahedron structures, where each mirror support point forms a node of the space frame (Fig. 31). The final choice will depend on structural stability, cost and efficiency of production. Stiffness requirements will depend on whether active mirror alignment is employed to partly compensate for dish deformations. This option is particularly interesting for the LST.

Construction materials The materials primarily used for the telescope structures are steel, aluminium and, more recently, carbon fibre reinforced plastic (CFRP). All have their advantages and drawbacks, particularly when building many telescopes at remote sites:

Steel Steel is the most commonly used material for past constructions, such as H.E.S.S. and VERITAS. It is generally the cheapest material, but results in rather heavy constructions. Nearly

Fig. 31 Sketch of the triangular space frame top layer with hexagonal mirror elements (blue lines). The mirror supports points (green circle) are fixed close to the space frame corners

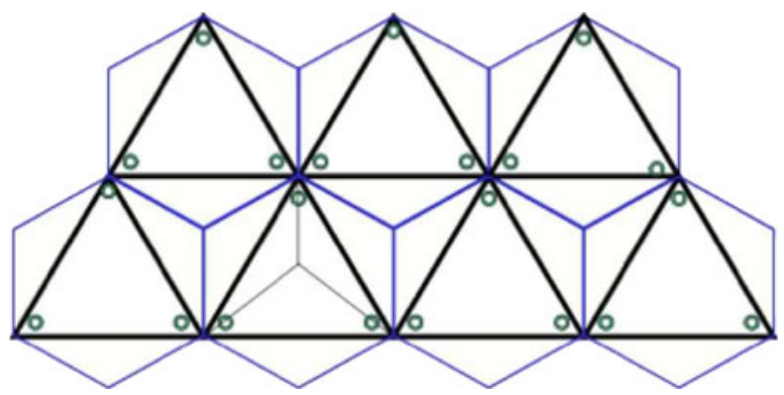


everywhere in the world expertise in steel fabrication and construction can be found.

Aluminium Aluminium is less heavy than steel and has a higher specific Young's modulus, but it has the largest thermal expansion of all three materials considered here.

CFRP CFRP is the strongest of the three materials and has the lowest weight, but it is the most expensive. It undergoes very little thermal expansion and is better as regards oscillation damping than the other materials, but connecting different elements is more difficult. This drawback might be overcome by an appropriate design, for example by use of composite-composite instead of metal-composite connections. CFRP is used in the MAGIC telescopes, to minimise their weight and moment of inertia to allow the maximum possible slewing speed.

\subsubsection{Current baseline designs}

For the MST and LST, the mechanically most complicated and costly structures, as well as for the SST, the following designs have emerged as baseline options (with other options still being pursued in parallel):

MST The general belief within the consortium is that the MST will become the workhorse of the CTA observatory. This implies that quite a number of telescopes will be built. Simplicity, robustness, reliability and the ease to maintenance are therefore particularly important features. This led to the decision to build an early prototype. MC studies suggest that an $f / d$ of around 1.4 and a FoV of about $8^{\circ}$ is required. Three groups within the consortium have developed their designs (Figs. 32 and 33).

The main idea in the first design was to have the elevation axis close to ground level. This solution saves on the construction of elevation towers, but at the expense of a pit into which the lower half of the dish disappears when the telescope is parked with the camera at ground level (Fig. 32, left). The same team is working on a design that decouples the dish movement from the camera elevation. The second design was based on a light and stiff dish, which consists solely of CFRP and is designed in a way that avoids CFRP joints to metal (Fig. 32, right). This design allows easy access to the camera and mirrors. For the elevation, two options were foreseen, a lift-up system and a more conventional swing-like mount.

The third design started from a mirror layout and a structural analysis. Two design options were considered: one has similarity with the H.E.S.S. I telescopes, the other with VERITAS. The second option with the central positioner has been worked out in more detail as this design simplifies of construction and reduces costs substantially (Fig. 33). A discussion between the three different design groups has started and has led so far to the use of the CFRP camera structure of the second design in the third design. All three designs are judged to be technically feasible, as a consequence of which the 

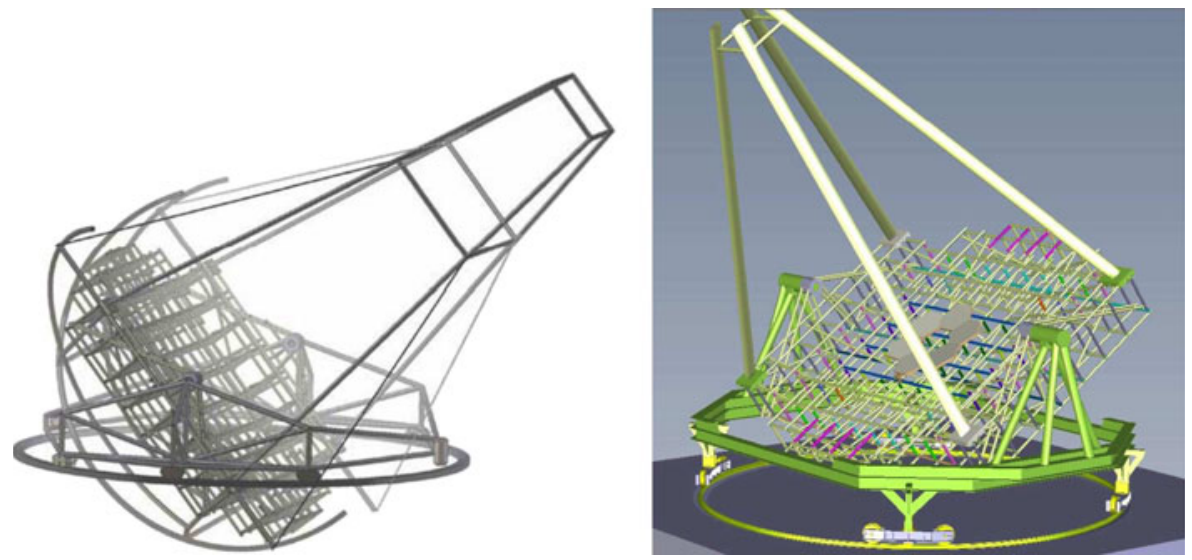

Fig. 32 Left Putting the telescope into a pit reduces the height of the telescope. Right A CFRP dish on a steel mount. In both cases the dish is held at the edge and the azimuthal movement is realised by rails

costs will be the major criterion of choice. After the decision on the design, a prototype will be constructed, probably next to an institute and not at the experimental site. The main aim of this prototype will be the optimisation and simplification of the instrument with respect to construction and maintenance.

Fig. 33 This design makes use of a positioner for the movement around the azimuthal and elevation axis

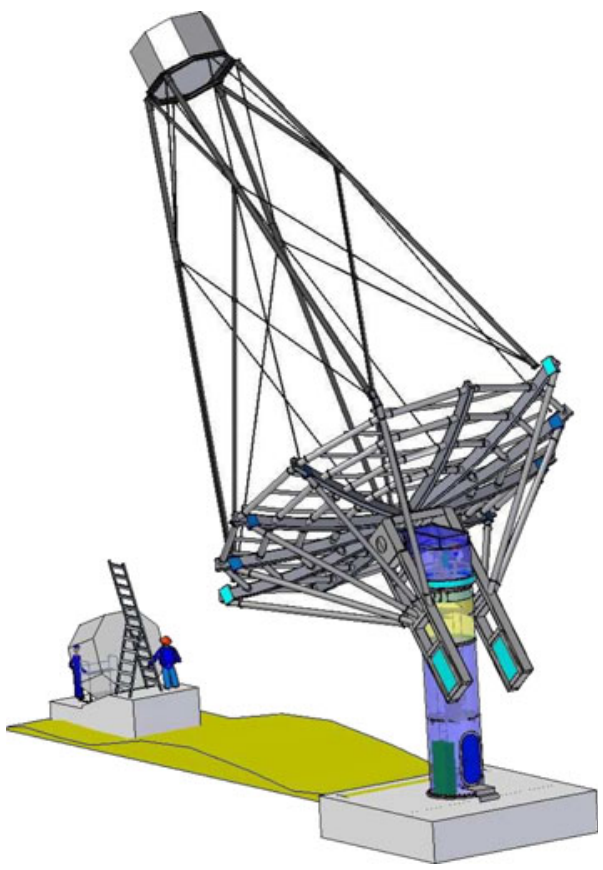

Springer 
Fig. 34 Model of an AGIS Schwarzschild-Couder telescope and its two-mirror aplanatic optical system (from [96])

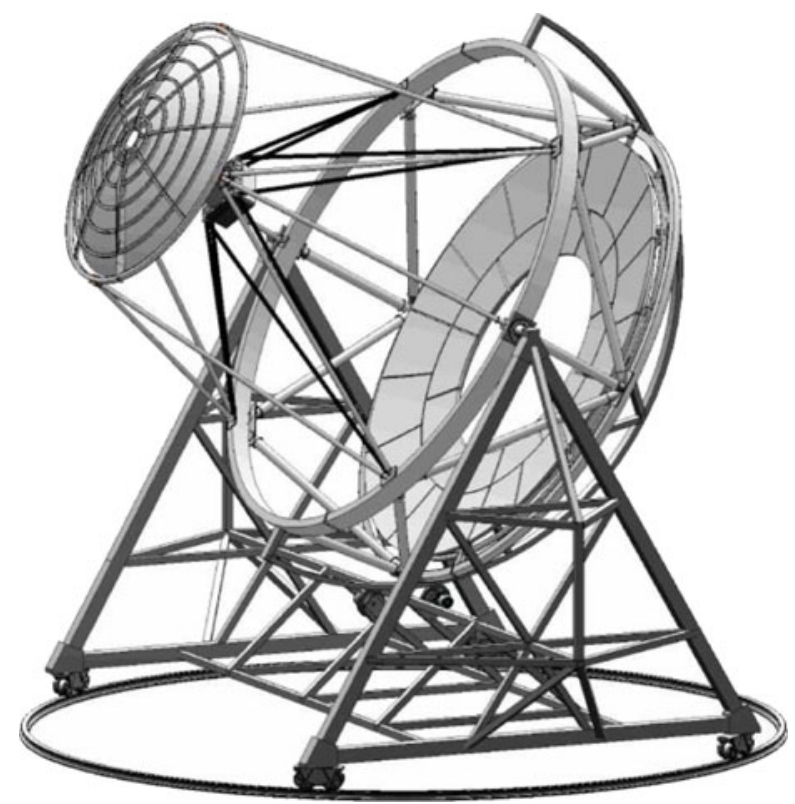

In parallel with the prototyping of the single-mirror MST, the design of a Schwarzschild-Couder telescope for AGIS has progressed (see Fig. 34) and work towards prototyping of components and ultimately a full MST-SC prototype is underway in the US.

LST For the LST, the current baseline design consists of a parabolic dish of 23 m diameter with $f / d=1.2$ constructed using carbon fibre structure (an enlarged derivative of the proven MAGIC design). The goal is to keep the total weight around $50 \mathrm{t}$ (Fig. 35). The dish uses a 3-or 4-layer space frame, based on triangular elements, with hexagonal mirrors supported from some of the nodes of the space frame. The dish is supported by an alt-azimuth mount moving on 6 bogeys along a circular rail.

SST For the SST, the mechanical design is less complex and several therefore timescales are somewhat more relaxed. Several options are still under study. The large FoV that is essential for the SST results, for single-mirror designs, in a relatively large camera with high costs. In comparison, the structure of the small telescope is cheap. This large misbalance makes it sensible to investigate an SST with a secondary optics which can potentially significantly reduce the camera cost, at the price of a more expensive mechanical structure. Whether this results is an overall saving is currently investigated. A possible design of a two-mirror system is shown in Fig. 36 (left). The design of a 6-m conventional telescope is pursued in parallel (Fig. 36, right). The costs of these two fundamentally different concepts are now being evaluated. The result will determine which SST design will be selected. 
Fig. 35 Conceptual layout of the LST. The dish has a diameter of $23 \mathrm{~m}$
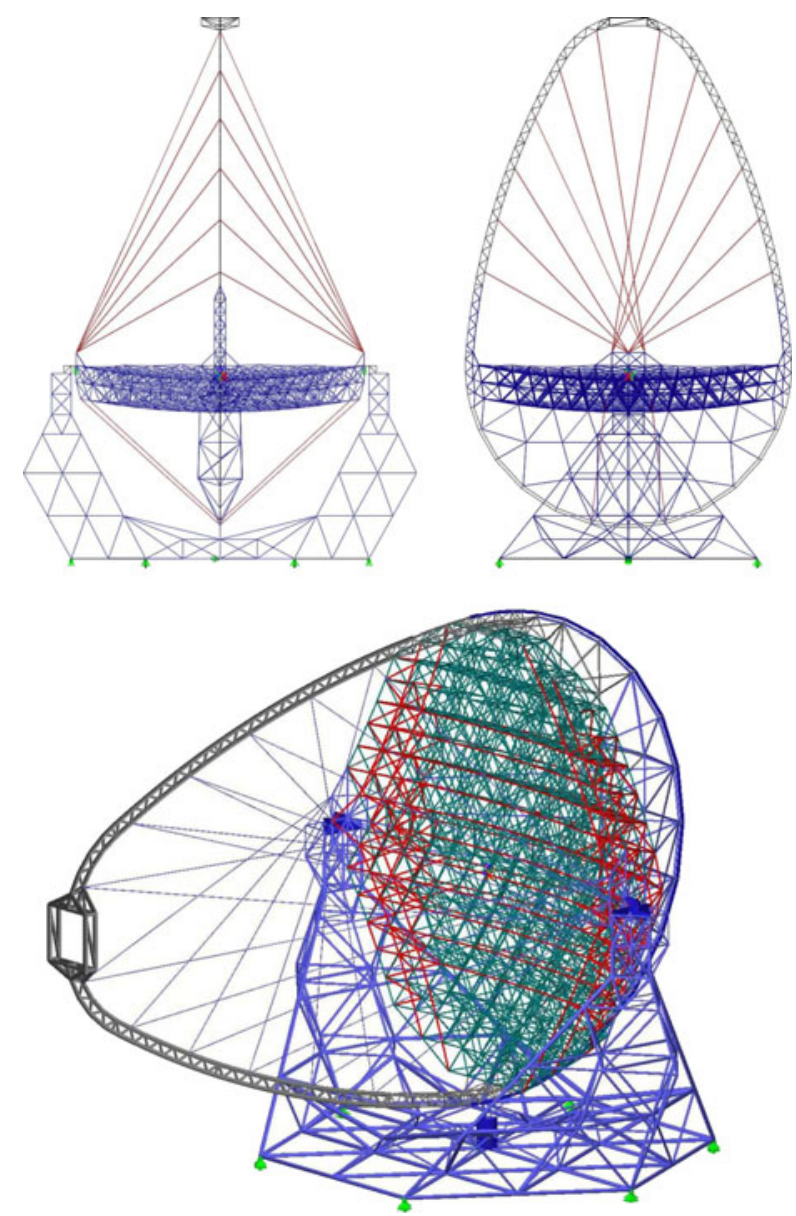

9.2 Telescope optics and mirror facets

\subsubsection{Telescope optics}

The reflector of each telescope images the Cherenkov light emitted by the air showers onto the pixels of the photon detection system. Apart from the total reflective area, which determines the amount of light that can be collected, the important parameters of the reflector system are:

The point spread function. The PSF quantifies how well the reflector concentrates light from a point source. The RMS width of the PSF should be less than half the pixel diameter for $40 \%$ containment if centred on a pixel, (for a Gaussian PSF), or better than $1 / 3$ of the pixel diameter for $68 \%$ containment. 

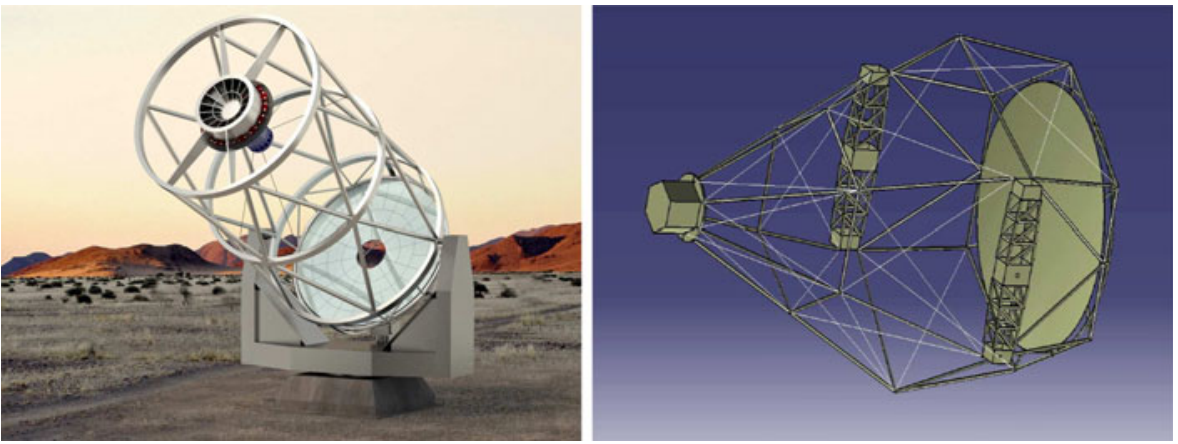

Fig. 36 Conceptual layouts of a small telescope. Left: two-mirror system; right: conventional onemirror system. The dish is held at the edge and the azimuth movement is realised by a central bearing

The time dispersion. Different light paths through the telescope results in a dispersion in the arrival time of photons on the camera, which should not exceed the intrinsic width of about $3 \mathrm{~ns}$ of the Cherenkov light pulse from a gamma-ray shower.

The reflector is usually segmented into individual mirrors. For the optics layout, most current instruments use either a parabolic reflector, which minimises time dispersion, or a Davies-Cotton design [100], where mirror facets of focal length $f$ (and hence radius of curvature $2 f$ ) are arranged on a sphere of radius $f$ (see Fig. 37), and which provides improved off-axis imaging. At the large field angles required for imaging Cherenkov telescopes, single-mirror designs suffer from significant optical aberrations with a resulting increase in

Fig. 37 Davies-Cotton mirror optics, with mirror facets of focal length $f$ arranged on a sphere of radius $f$

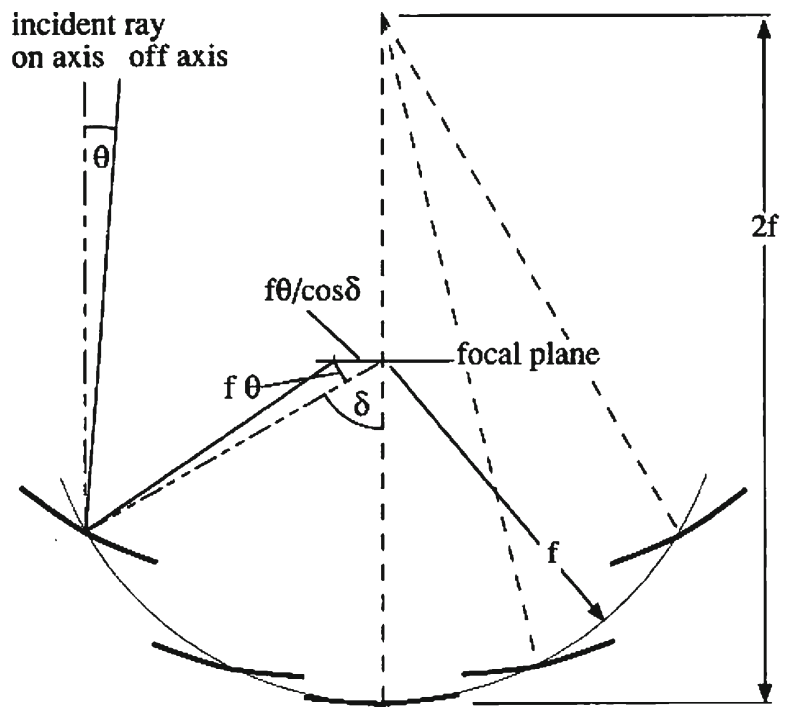


PSF. Dual-mirror designs can provide significantly improved imaging, at the expense of a more complex telescope design [66].

For a parabolic reflector of diameter $d$, focal length $f$ and focal ratio $F=$ $f / d$, the RMS width of the PSF can be approximated by [65]

$$
\sigma_{\zeta}^{2}=\frac{1}{512} \frac{\delta^{2}}{F^{4}}+\frac{1}{16} \frac{\delta^{4}}{F^{2}} \quad \text { and } \quad \sigma_{\eta}^{2}=\frac{1}{1536} \frac{\delta^{2}}{F^{4}}
$$

where $\delta$ is the field angle and $\sigma_{\zeta}$ and $\sigma_{\eta}$ are the widths of the PSF in the radial and azimuthal directions, respectively. The spot size is always larger in the radial direction, mostly due to the non-Gaussian tails of the PSF. For a parabolic reflector, the two spot dimensions differ by a factor of more than 1.7, resulting in systematic distortions of Cherenkov images for off-axis sources.

For a Davies-Cotton reflector with a planar focal surface, the corresponding expressions are [66]

$$
\begin{aligned}
\sigma_{\zeta}^{2} & =\frac{1}{1024} \frac{\delta^{2}}{F^{4}}\left(1-\frac{1}{4 F^{2}}\right)+\frac{1}{256} \frac{\delta^{4}}{F^{2}}\left(4+\frac{35}{6 F^{2}}\right) \quad \text { and } \\
\sigma_{\eta}^{2} & =\frac{1}{1536} \frac{\delta^{2}}{F^{4}}\left(\frac{10}{9}+\frac{9}{32 F^{6}}\right) .
\end{aligned}
$$

The difference between the radial and azimuthal spot sizes is less pronounced in this case, typically around $20 \%$. The Davies-Cotton design results in a flat distribution of photon arrival times, with a maximum time difference of $D /(8 F \cdot c)$, and an RMS time dispersion $\sigma_{t}=d /(16 \sqrt{3} F \cdot c) \approx 0.12 d / F \mathrm{~ns} / \mathrm{m}$.

Usually, the first term in the expansions for the PSF dominates, resulting in a roughly linear increase of the PSF with the field angle $\delta$, and a quadratic dependence on $F$. For typical parameter values, $\sigma_{\zeta}$ is $20-30 \%$ smaller for the Davies-Cotton design than for a parabolic mirror, whereas $\sigma_{\eta}$ values are similar.

The expressions given above assume perfect shapes of the mirror facets, and very small facets for the Davies-Cotton design. In real applications, individual mirror facets will have an intrinsic spot size, which to a first approximation must be added quadratically to the PSFs given above. Parabolic mirrors can be constructed using spherical facets with focal lengths that are adjusted in 2-3 steps, rather than varying continuously according to their radial position. The optimal radii $r_{1}$ and $r_{2}$ for aspherical mirrors at a distance $R$ from the optical axis of a parabolic dish of focal length $f$ are

$$
\frac{r_{1}}{2 f}=\sqrt{1+\frac{R^{2}}{4 f^{2}}} \approx 1+\frac{R^{2}}{8 f^{2}} \quad \text { and } \quad \frac{r_{2}}{2 f}=\sqrt{\left(1+\frac{R^{2}}{4 f^{2}}\right)^{3}} \approx 1+\frac{3 R^{2}}{8 f^{2}} \text {. }
$$

Use of spherical facets will cause a typical contribution to the spot size of order $(d / 8 f)^{2}$, equivalent to that caused by the typical spread of $1 \%$ in facet focal length. Effects on the PSF are hence modest. The same holds for the influence of the facet size in the Davies-Cotton layout, as long as the number of facets is still large. Figure 38 illustrates how the PSF varies across the field of view, for different values of $f / d$, based on a realistic Monte Carlo simulation, including 

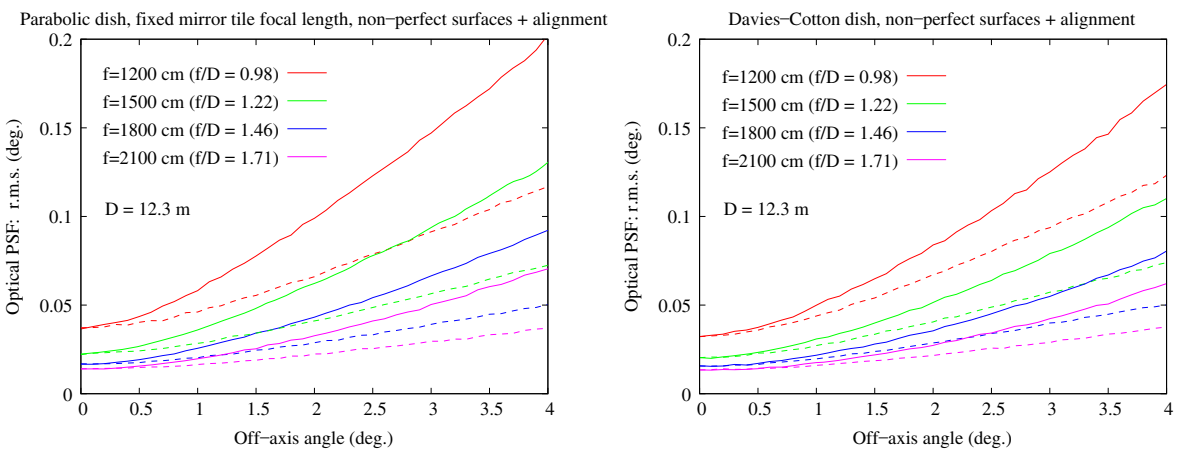

Fig. 38 PSF (RMS) as a function of field angle, for a parabolic dish of different $f / d$ (left) and for a Davies-Cotton dish (right). Full lines represent the radial component of the PSF, dashed lines the transverse component

the effects of the PSF of the individual mirrors, the alignment inaccuracy, and the use of spherical mirror facets for the parabolic reflector.

For the SST and MST, among single-reflector designs a Davies-Cotton geometry provides the best imaging over a large field of view. For the LST only a parabolic dish is possible due to the large time dispersion a DaviesCotton design would introduce. To achieve a PSF of $3^{\prime}$ over a $7^{\circ}$ field of view, an $F$ value of about 1.5 is required.

Dual-mirror telescopes have so far not been used in Cherenkov astronomy, but obviously allow improved compensation of optical errors over a wide field of view. In [66] dual-reflector designs are discussed in depth, with particular emphasis on the Schwarzschild-Couder design which combines a small plate scale (adapted to the use of multi-anode PMTs as photo-sensors) with a $3^{\prime}$ PSF across a $5^{\circ}$ radius field of view (see Fig. 39). Compared to single-reflector designs, where the camera has to be supported at a large distance $F \cdot d$ from
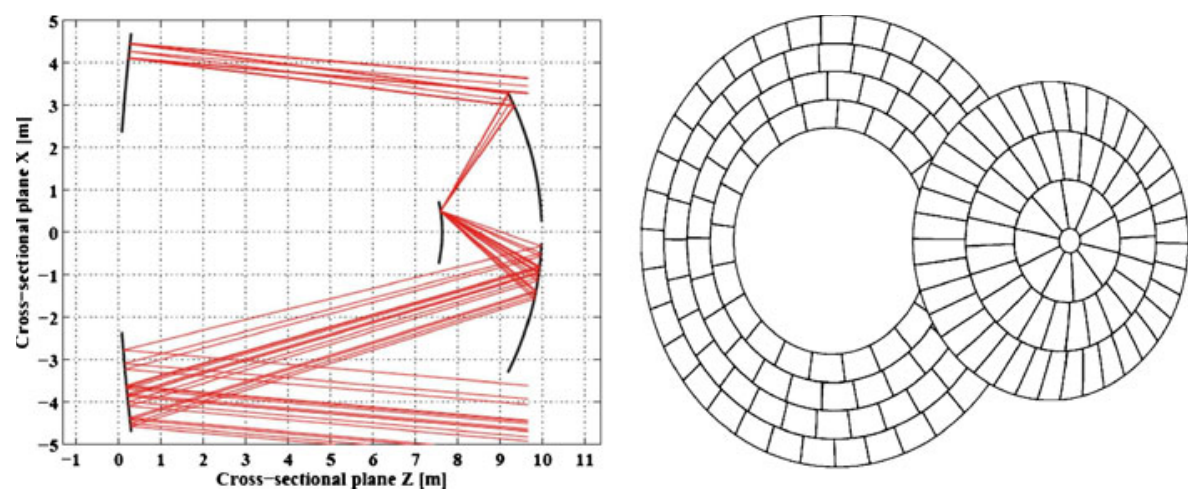

Fig. 39 Dual-reflector optics design for Cherenkov telescopes providing an improved PSF over a large field of view combined with a small plate scale [66] 
the dish, the dual-reflector design is quite compact. Drawbacks include the fact that non-spherical mirrors are needed, which are more difficult to fabricate, and that the tolerances on the relative alignment of optical elements are rather tight. Also, the large secondary reflector results in significant shadowing of the primary reflector. CTA's US collaborators, together with some European groups, plan to build a Schwarzschild-Couder telescope of $12 \mathrm{~m}$. While current CTA designs are based on single-reflector telescopes, a dual-reflector construction could be adopted in particular for the SST or the MST, should the developments prove promising.

To realise the PSFs given above, obviously the orientation of the mirror facets has to be stable to a fraction of the PSF under varying dish orientations, temperatures, temperature gradients, and wind loads. Due to the reflection, orientation errors enter with a factor of 2 into the PSF. The facet orientation can be stabilised either by using a rigid dish, or by active compensation of dish deformations. For example, the mechanical structure of the H.E.S.S. telescopes is designed to keep the facet orientation stable to within $0.14 \mathrm{mrad}$ $\left(0.5^{\prime}\right)$ RMS over the elevation range $45-90^{\circ}$ and the operational range for wind loads and temperatures [101]. In MAGIC, an active mirror alignment system compensates for dish deformations [102]. The initial alignment of the mirror facets, as well as the calibration of active systems, is usually carried out using images of bright stars and has been demonstrated to have a precision well below the typical 3' PSF (e.g. [83]).

Of additional interest is the precision with which the real dish shape needs to approximate the ideal shape. Use of straight beam segments to approximate a curved dish may simplify production considerably. Two effects matter: an otherwise ideal facet displaced by $\delta z$ along the optical axis will generate a spot of angular diameter $\Delta \zeta=d_{\text {facet }} \delta z / f^{2}$ where $d_{\text {facet }}$ is the facet diameter. The corresponding RMS is $\sigma_{\zeta}=\Delta \zeta / 4$. Typically, facets have a PSF of $1 \mathrm{mrad}$ diameter or better. Limiting additional contributions due to imperfect facet placement to $1 \mathrm{mrad}$, which implies that they matter only near the centre of the field of view, where the facet PSF dominates imaging, one finds $\delta z<$ $10^{-3} f^{2} / d_{\text {facet }}$, or $0.2 \mathrm{~m}$ for $f=15 \mathrm{~m}$ and $d_{\text {facet }}=1 \mathrm{~m}$. If the focal distance is wrong for a given facet, the spot location for off-axis rays will also be shifted by $\Delta \zeta=\delta \Delta z / F$, which should again be small compared to the spot size, for typically requiring $\Delta z<0.1 \mathrm{~m}$. Another limit comes from the time dispersion introduced by this deviation, which is $\Delta T=2 \Delta z / c$, implying that $\Delta z$ should not exceed $0.1-0.2 \mathrm{~m}$. In summary, mirror placement along the optical axis should be within $10 \mathrm{~cm}$ of the nominal position for the MST.

\subsubsection{Mirror facets}

Because of its large size, the reflector of a Cherenkov telescope is composed of many individual mirror facets. It is therefore important to balance the ease and cost of production techniques against the required optical precision. In total, CTA will need of the order of $10^{4} \mathrm{~m}^{2}$ of mirror area, an order of magnitude more than current instruments. As the telescopes are required to 
observe the Cherenkov light emitted from the many particle tracks of an extensive air shower, the necessary optical precision of the mirror system is relatively relaxed. Focusing can be worse than is required for mirrors for optical astronomy by about two orders of magnitude, and the distance of the mirror facets to the focal plane needs to be correct only to within a few $\mathrm{cm}$, as opposed to the sub-wavelength precision needed in optical astronomy.

The mirror facets for CTA will probably have a hexagonal shape and dimensions of $1-2.5 \mathrm{~m}^{2}$. Large mirror facets have the advantage that they reduce the number of facets on a dish and the number of support points and alignment elements required. On the other hand, in particular for DaviesCotton optics, the optical performance worsens as mirror facets become larger. Also, the choice of manufacturing technologies becomes rather limited. For these reasons, the current baseline for the MST is to use hexagonal mirrors of $1.2 \mathrm{~m}$ (flat-to-flat) diameter. Performance criteria for facets are equivalent to those for current instruments as regards the spot size, the reflectance and requirements on the long-term durability. The reflected light should largely be contained in a $1 \mathrm{mrad}$ diameter area, the reflectance in the $300-600 \mathrm{~nm}$ range must exceed $80 \%$, and facets must be robust against ageing when exposed to the environment at the chosen site for several years. Spherical facets are in most cases a sufficiently accurate approximation. For a parabolic dish, a variation of facet focal length with distance from the dish centre may be considered, although gains are modest for a dish with relatively large $f / d$.

Several technologies for the production of mirror facets for Cherenkov experiments were used in the past, or are under development at present. These can be divided into two classes: technologies using grinding/polishing or milling of individual mirrors, as used for most current instruments; and replication techniques, where mirrors are manufactured using a mould or template, which has obvious advantages for mass production.

Facet types produced using grinding or milling techniques include:

Glass mirrors which have been the standard solutions for many past and present Cherenkov telescope (e.g. HEGRA, CAT, H.E.S.S., VERITAS). The mirrors were produced from machined and polished glass blanks that were front-coated in vacuum with aluminium and some weather-resistant transparent protection layer, such as vacuum deposited $\mathrm{SiO}_{2}$ (HEGRA, CAT, H.E.S.S.), or alternatively $\mathrm{Al}_{2} \mathrm{O}_{3}$ applied by anodisation (VERITAS). These mirrors exhibit high reflectivity and good PSFs and there is extensive production experience. Drawbacks are their fragility and weight, in particular if facets of $\geq 1 \mathrm{~m}^{2}$ are considered. Their front-side coating shows relatively fast ageing and degradation when exposed permanently to the wind and weather. A typical degradation of the reflectance of around $5 \%$ per year is observed for a single $\sim 100 \mathrm{~nm} \mathrm{SiO} 2$ protection layer. Production and handling of thin (few $\mathrm{cm})$ and large $\left(1 \mathrm{~m}^{2}\right)$ facets is nontrivial.

Diamond-milled aluminium mirrors are used in the MAGIC telescopes [103]; these light-weight mirrors are composed of a sandwich of two thin 
aluminium layers, separated by an aluminium hexcell honeycomb structure that ensures rigidity, high temperature conductivity and low weight (see Fig. 40). After a rough pre-milling that ensures approximately the right curvature of the aluminium surface, the mirror is precisely machined using
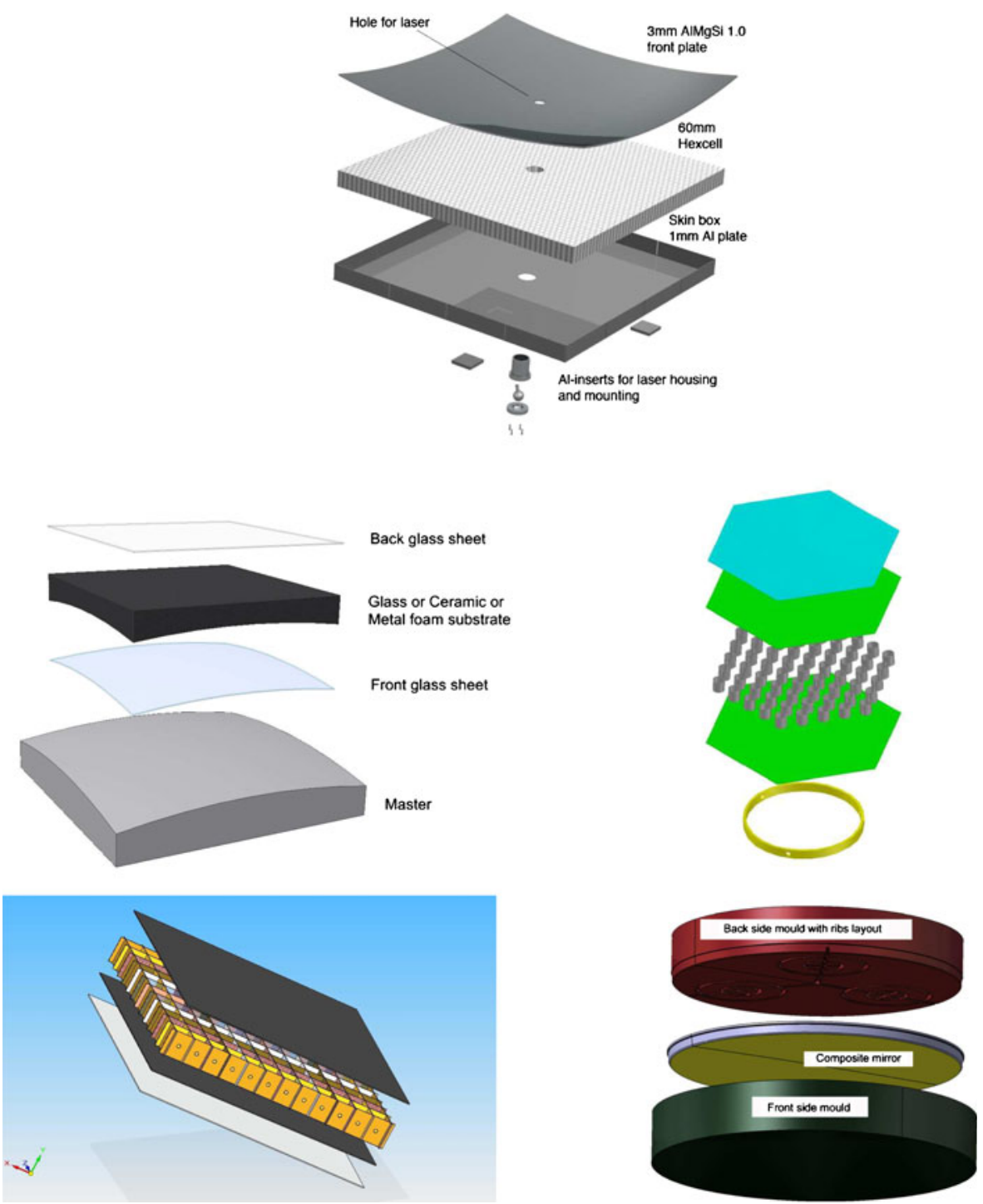

Fig. 40 Various mirror types under consideration for CTA. Top: Diamond-milled aluminium honeycomb mirrors. Middle left: Cold slumped glass-foam sandwich mirrors. Middle right: Open fibre-reinforced plastics mirror (carbon fibre or glass fibre). Lower left: Carbon-fibre composite mirror with CFRP honeycomb. Lower right: Carbon-fibre composite mirror produced with SMC technology 
diamond-milling techniques. A thin layer of quartz of $\sim 100 \mathrm{~nm}$ thickness, with some carbon admixture, is plasma coated on the mirror surface for protection against corrosion. Diamond-milled mirrors have proven more resistant to ageing effects (reflectance loss of $1-2 \%$ per year) than mirrors with a thin reflective coatings on glass or other substrates, presumably since the reflective layer cannot be locally destroyed. On the other hand, the initial reflectance of diamond-milled mirrors is a few percent lower.

An ongoing development is the mass production of mirror panels by means of replication technologies. These are cost effective and can be used to produce non-spherical and very light-weight mirrors with good and reproducible optical quality. Replication methods look to be promising for the large-scale production of CTA mirror facets and will be considered as the baseline design, although long-term tests are still required. Replica production methods include:

Cold slumped glass mirrors. The mirror panels are composed of two thin glass sheets $(1-2 \mathrm{~mm})$ glued as to a suitable core material, giving a structure with the necessary rigidity. Construction proceeds as follows: At room temperature, the front glass sheet is formed to the required optical shape on a master by means of vacuum suction. The core material and the second glass sheet are glued to it. After the curing of the glue, the panel is released from the master, sealed and coated in the same way as a glass mirror. Half of the mirrors of the MAGIC II telescope were produced with this technology using an aluminium honeycomb Hexcell structure as core material [104]. For CTA, other core materials are under investigation, such as various foams. Especially promising is an all-glass closed-cell foam that can be pre-machined to the required curvature (see Fig. 40). Further investigation of the effects of thermal insulation between the front and the back of the mirror caused by foams is required.

Aluminium foil mirrors. Aluminium honeycomb sandwich mirrors with reflective aluminium sheets of $1-2 \mathrm{~mm}$ thickness (made e.g. by the company Alanod) are also being studied in detail. Their main limitation currently results from the imperfect reflection properties of the aluminium foil.

Fibre reinforced plastics mirrors. Several attempts are being made to use carbon- or glass-fibre reinforced plastic materials to produce light-weight mirror facets. Three different technologies are currently under development for CTA: (a) an open sandwich structure of glass-fibre or carbonfibre reinforced plastic, consisting of two flat plates and spacers with either an epoxy layer cast on one plate or a bent thin glass sheet glued to it to form the mirror surface; (b) a closed structure of two carbon-fibre reinforced plastic plates bent to the required radius of curvature, an intermediate pre-machined CFRP honeycomb for stability and a thin glass sheet as reflecting surface; and (c) a one-piece design using a compound containing carbon-fibre and the high-temperature and high-pressure sheet moulding (SMC) technology, which is frequently used in the automotive industry. 
To form a smooth surface in the same production step an in-mould coating technology is under investigation which would allow for production times of the order of just a few minutes per substrate. See Fig. 40 for the different mirror types.

Since the mirrors are permanently exposed to the environment, degradation of mirror reflectivity is a serious concern. In the case of aluminium-coated mirrors, water can creep along the interface of the glass and aluminium layer because the aluminium does not stick perfectly to the glass surface and the protective layers often have pin holes. In contrast, solid aluminium mirrors show localised corrosion, which, even when deep, affects only a very small fraction of the surface. Possible cures for the glass mirrors could be intermediate layers improving adhesion, e.g. of chromium or $\mathrm{SiO}$, or more resistant protection layers, for example multiple layers which reduce the probability of pin holes in the coating. Multi-layer protective coatings could also be used to enhance reflectivity in the relevant wavelength region. These are under investigation as are purely dielectric coatings without any aluminium, which consist of multiple layers with different refractive indices. These latter can in principle provide reflectances of up to $98 \%$ and would not suffer from the rather weak adhesion of aluminium to glass.

Another option to improve the mirror lifetime is to apply the reflective coating to the protected back side of a thin glass sheet, which could then be used in the replication techniques described above. Disadvantages are transmission losses in the glass, the requirement of a very uniform glass thickness, as the mould defines the shape of the front side but the reflective layer is on the back, and, in addition, icing problems due to radiation cooling of the front surface.

In summary, many different technologies for the production of mirror facets are under investigation. For several of them, large-scale production experience exists already, others are in a development phase. A challenge in mirror production will be to find the optimum compromise between mirror lifetime and production costs. Current production costs are $1,650 € / \mathrm{m}^{2}$ for the $0.7 \mathrm{~m}^{2}$ H.E.S.S. II glass mirrors, $2,450 € / \mathrm{m}^{2}$ for the $1.0 \mathrm{~m}^{2}$ MAGIC II milled-aluminium mirrors, and $2,000 € / \mathrm{m}^{2}$ for the $1.0 \mathrm{~m}^{2}$ MAGIC II coldslumped glass mirrors. The much larger production scale of CTA and the use of optimised techniques is expected to result in a significant reduction in cost, in particular for the replication technologies. Current baseline specifications for MST mirror facets are summarised in Table 2.

Table 2 Baseline specifications for mirror facets (MST)

\begin{tabular}{ll}
\hline Shape & Hexagonal \\
Size & $1,200 \mathrm{~mm}$ flat-to-flat \\
Type & Spherical (aspherical opt.) \\
Focal length & $\sim 16 \mathrm{~m}$ \\
Reflectance & $>80 \%$ between 300 and $600 \mathrm{~nm}$ \\
Spot size & $<1 \mathrm{mrad}$ diameter $(80 \%$ containment $)$ \\
\hline
\end{tabular}




\subsubsection{Mirror support and alignment}

To achieve design performance, mirror facets need to be aligned with a precision which is about an order of magnitude better than the optical point spread function, i.e. given the PSF requirement of $<1 \mathrm{mrad}$ the alignment precision needs to be well below $0.1 \mathrm{mrad}$ or $100 \mu \mathrm{m}$ (assuming a typical $1 \mathrm{~m}$ lever arm between mirror support points). Various alignment methods are in use for existing telescopes:

Manual alignment. Using an appropriate adjustment mechanism, mirror facets are manually aligned after mounting. For technical reasons, alignment is usually performed at or near the stow position of the telescope. Deformations in dish shape between the stow position and the average observation position (at $60-70^{\circ}$ elevation) can be compensated by "misaligning" mirrors by the appropriate amount in the stow position. This scheme is used in the VERITAS telescopes [105].

Actuator-based alignment. Initial alignment of mirrors is carried out by remote-controlled actuators, using the image of a star viewed on the camera lid by a CCD camera on the dish, and implementing a feedback loop which moves all facet spots to a common location. This scheme is employed by H.E.S.S. [83].

Active alignment. Remote-controlled actuators are used not only for initial alignment of facets, but also to compensate for deformations of the dish, in particular as a function of elevation. If dish deformations are elastic, reproducible and not very large compared to the point spread function, alignment corrections can be based on a lookup table of actuator positions as a function of telescope pointing. If deformations are large or inelastic, a closed feedback loop can be implemented by actively monitoring facet pointing, using lasers attached to each facet and imaged onto a target in the focal plane. Active alignment is used by MAGIC [102].

Technically, the requirements for the actuator-based alignment and the active alignment are very similar, the main difference being that for active alignment of a significant fraction of facets need to be moved simultaneously or nearly simultaneously as telescope pointing changes, requiring parallel rather than serial control of actuators and a higher-capacity power supply. Since manual alignment of the large number of CTA mirror facets is impractical, certainly the medium-sized and large telescopes will be equipped with actuators. The small and medium sized telescopes will have mechanically stable dish structures which do not necessarily require active control, but active (look-up table driven) mirror control could be implemented to maintain optimum point spread function over the entire elevation range.

Desirable features for actuators include a movement range of at least $30 \mathrm{~mm}$ and a built-in relative or, better, absolute position encoder which allows the actuator to be moved by an exact pre-defined amount. This is particularly relevant for lookup-based corrections. For active alignment, the positioning 
speed needs to be such, that the changes in mirror alignment are performed within the time needed to move the telescope to a different position. For actuator based alignment it is sufficient to be able to perform an initial alignment within a few days and possible re-alignments within a few hours. When not moving, actuators should be self-blocking to avoid movements e.g. in the case of power failure. The actuators need to perform reliably and without significant maintenance over the expected lifetime of the CTA array of over 20 years. (The mean time between failure (MBTF) should be 100 years.)

Figure 41 shows a prototype actuator design based on a spindle driven by a stepper motor, with a combination of a digital Gray-code rotation encoder and analogue signals from four Hall probes providing absolute position sensing. The actuator is controlled by wireless communication using the Zigbee industry standard, with each actuator identified by a unique (48 bit) code. A broadcast mode is also available, which could be used to communicate the current elevation to all actuators allowing the controller to look up and apply the relevant individual correction values.
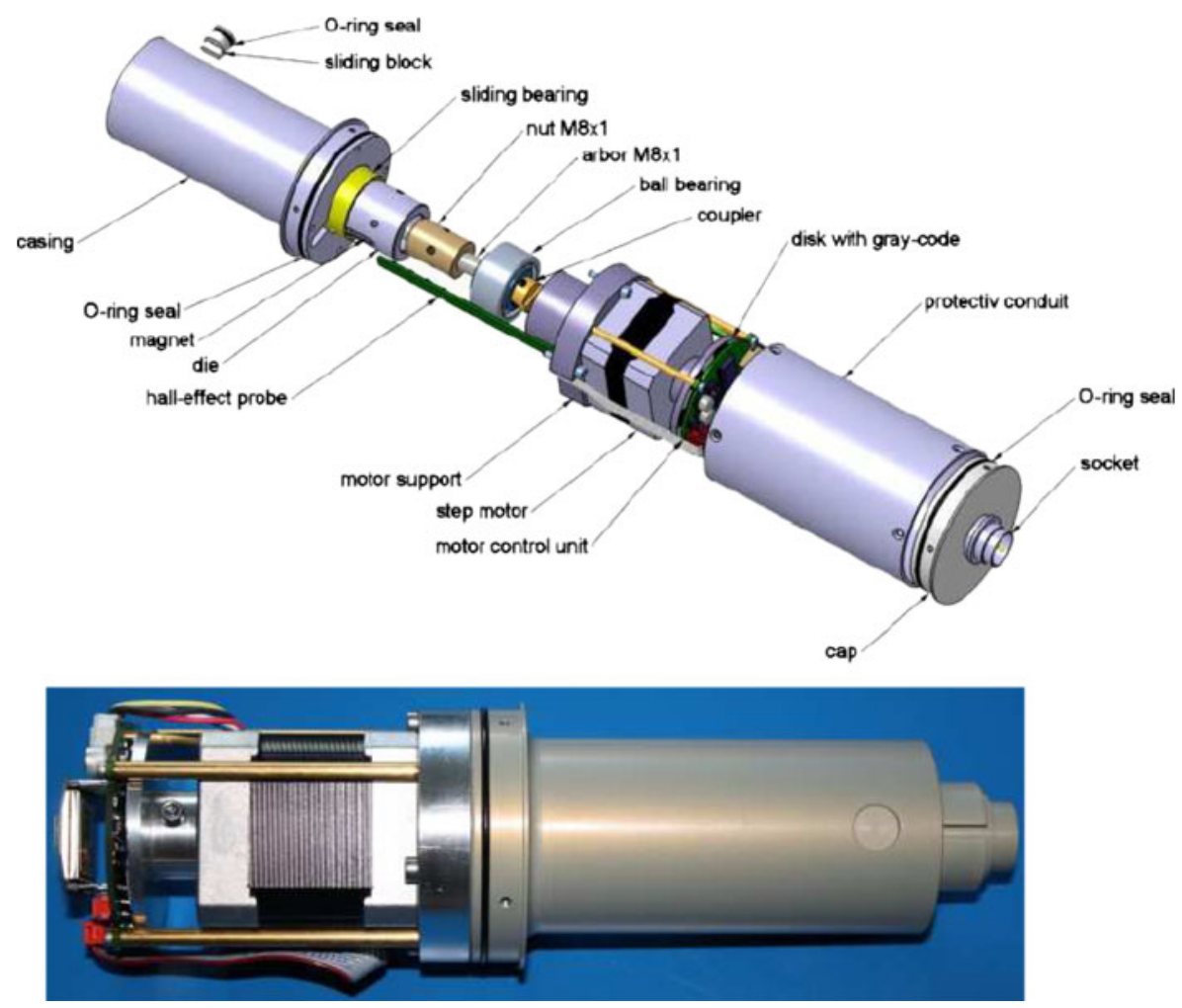

Fig. 41 Prototype mirror actuator based on a stepper-motor driven spindle, providing absolute position encoding and a wireless control interface 
A second solution can be seen in Fig. 42. The upper part of the figure shows the motor, two actuators, and the micro-controller board of one mirror unit. This device uses servo motors with a Hall sensor attached to the motor axis which makes possible relative positioning of the actuator with high accuracy. The communication is based on CAN (Controller Area Network), a multi-master broadcast serial bus standard which is used in the automotive industry and other areas where there is demand for high reliability. The communication of the telescope units with the control computer is done via Ethernet. The electronics layout is depicted in the lower part of the figure.

Mirror facets will be attached at three points, two equipped with actuators and one universal joint. The facet mounting scheme should allow the installation of the facets from the front, without requiring access from the space-frame side of the dish. This can be achieved by supporting mirrors at the outer circumference, where attachment points are easily accessible, or using screws or attachment bolts going through the mirror. Current

Fig. 42 Upper part: Prototype mirror control actuators, motor, and micro-controller board for the solution based on relative position encoding. Lower part: Electronics layout of the setup

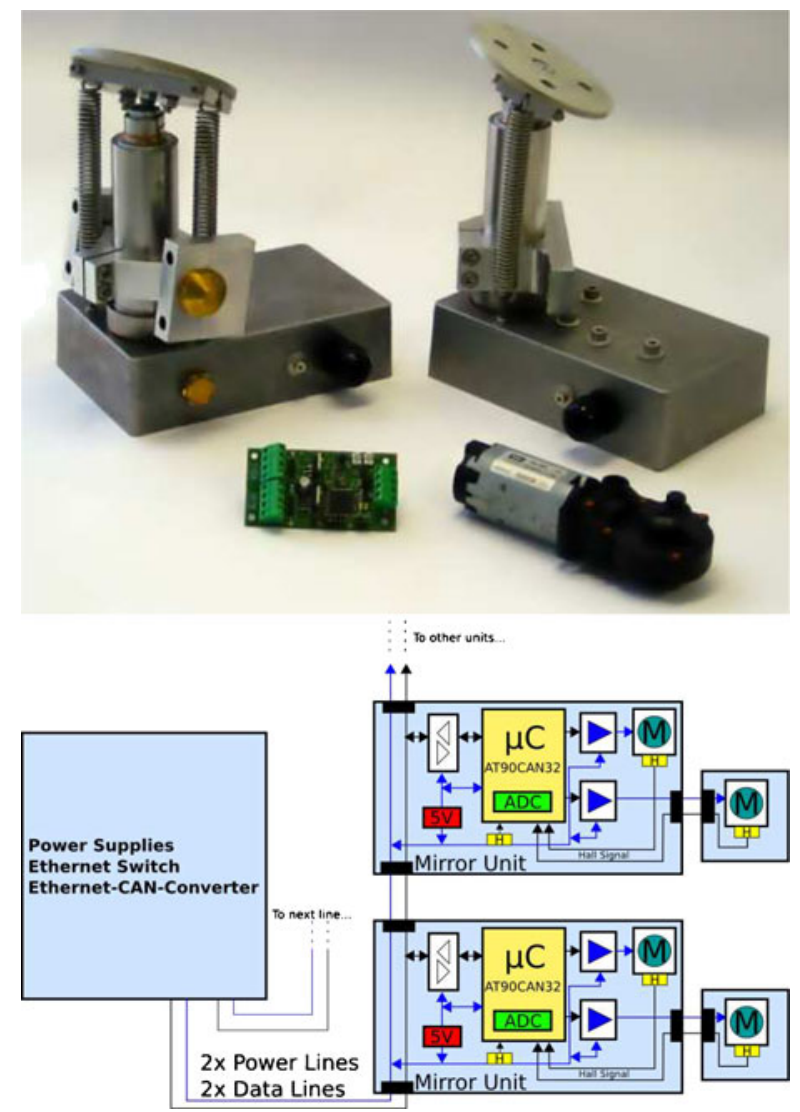


Table 3 Baseline specifications for mirror alignment actuators

Precision

Initial alignment

Re-alignment (actuator based alignment)

Re-alignment (active alignment)

Lifetime
$<0.1 \mathrm{mrad}$

$<$ few days

$<$ few hours

$<$ slewing time to new position

$>20$ years

baseline specifications for the mirror alignment system are summarised in Table 3.

\subsection{Photon detection, electronics, triggering and camera integration}

The cameras developed for gamma-ray detections with current atmospheric Cherenkov telescopes have reached the sensitivity required to perform detailed investigations of many astrophysical sources. Further advancing Cherenkov telescope performance requires, in particular, that the energy range covered be extended, i.e. that the gamma-ray energy threshold be reduced and detection capabilities be extended at high energies, enhancing the flux sensitivity, and improving angular resolution and particle identification. Lowering the threshold energy and increasing the sensitivity of an IACT requires that more Cherenkov photons be collected and/or that these are detected more efficiently. The efficiency of the collection of Cherenkov photons and their conversion to photoelectrons in the photo-sensor must therefore be improved; the non-sensitive regions (dead areas) in the camera must be minimised, for example by using light guides, the effective photon conversion efficiency increased by exploiting novel technical developments. Enlarging the energy range requires appropriate electronics with a sufficiently large dynamic range.

Achieving the required performance necessitates the development and the production of electronics components dedicated to CTA. Sophisticated application-specific integrated circuits (ASICs) for equipping the front-end part of the readout chain are under study. These have the advantage that they minimise signal distortion, decrease the power consumption and ultimately reduce the cost of the experiment considerably. Integrated readout systems take advantage of the recent development of analogue memories for data buffering. An alternative solution is a fully digital readout scheme. The amplified signal from the photon sensors is directly digitised by an analogueto-digital converter (ADC) and buffered in a deep memory. Readout and triggering benefit from continuous data storage to avoid deadtime.

The integration of detectors and the associated electronics reduces the size of the apparatus and embedded cameras have operational advantages, particularly at an isolated site and given the number of about one hundred cameras that will be required for CTA. The usage of complete spare cameras, rather than spare components for these cameras, can significantly simplify the maintenance of the system. The camera typically consists of a cylindrical 
structure built completely from low mass components. It holds a matrix of photon sensor cells, carefully optimised to make maximal use of the incoming light, and is fixed to the arms of the telescope in the focal plane, above the dish of the telescope. For embedded cameras, the light sensor, readout and trigger system, data acquisition system and power supply are integrated in a modular mechanical structure. The only connections that enter the camera are the input power, the communication network, and any central trigger cables. Disadvantages are a heavy camera requiring considerable cooling power and a heavy camera support structure.

\subsubsection{Photon detection}

The photon sensors most commonly used in IACTs are photomultipliers with alkali photo cathodes and electron multipliers based on a chain of dynodes. The technology is well-established, but is subject to continuous development and improvement. PMTs have established themselves as the best available low light level sensors for ultra-fast processes. The relatively high peak quantum efficiency (QE) currently available (up to 30\%), together with high gains of up to $10^{6}$ and low noise, allow the reliable measurement even of single photoelectrons. A dynamic range of about 5,000 photoelectrons is obtainable with PMTs. The PMTs convert impinging photons into a charge pulse of size measured in number of photoelectrons. IACTs usually use PMTs with bialkali type photo-cathodes, as these provide the highest QE. They are sensitive in the wavelength range of 300-600 nm (200-600 nm if a PMT with a quartz window is used). The bialkali PMT sensitivity curve is well-matched to the spectrum of Cherenkov light arriving at ground level from air showers. As a rule, one needs to amplify this pulse in order to match the sensitivity of the data acquisition (DAQ) electronics. However, new photon detectors are under study and the CTA cameras must be designed to allow their integration if their performance and cost provide significant advantages over PMTs.

\section{Criteria for photo-detectors}

Spectral sensitivity The spectrum of Cherenkov light is cut off below $300 \mathrm{~nm}$, due to atmospheric transmission effects, and falls off as $1 / \lambda^{2}$ towards longer wavelengths. ${ }^{5}$ Candidate photo-detectors should be matched to the peak in this spectrum at around $350 \mathrm{~nm}$. At large wavelengths, beyond about $550 \mathrm{~nm}$, the signal-to-noise ratio becomes increasingly unfavourable due of the increasing intensity of the night sky background in this region. Above $\sim 650 \mathrm{~nm}$ strong emission lines are present in the Cherenkov spectrum, originating from the rotational levels of $(\mathrm{OH})$ groups. It is therefore desirable but not essential to measure up to wavelengths of about

\footnotetext{
${ }^{5}$ Due to Rayleigh and Mie scattering the actual spectrum at the camera is considerably flatter (see Fig. 43). At large zenith angles the UV part of the spectrum might no longer be detectable.
} 
$600-650 \mathrm{~nm}$. (The more accurately the absolute charge in an image is measured, the better the absolute calibration.)

Sensor area Currently favoured pixel sizes are around $0.1^{\circ}$ for the LST, $0.18^{\circ}$ for the MST, and $0.25^{\circ}$ for the SST. For conventional telescope designs (single mirror optics, with Davies-Cotton or parabolic reflectors), these angular sizes translate to linear dimensions of 40,50 and $35 \mathrm{~mm}$, respectively. If a secondary-optics design is used for the SST, a size of $0.2^{\circ}$ represents around $6 \mathrm{~mm}$. For the secondary-optics design for the MST, a smaller angular pixel size of $0.07^{\circ}$ equates to the same physical size of $6 \mathrm{~mm}$. Light-collecting Winston cones in front of any sensor reduce the required sensor size by a factor of 3-4 compared to the pixel size and can decrease the amount of dead space between pixels.

Sensor uniformity Sensor non-uniformities below $\sim 10 \%$ are tolerable. Larger non-uniformities should be avoided as they introduce an additional variable component in the light collection and thus increase the variance of the output signal.

Dynamic range and linearity Sensors should be able to detect single photons and provide a dynamic range of up to 5,000 photo-electrons, with linearity deviations below a few per cent. Non-linearities can be tolerated if they can be accurately corrected for in the calibration procedure.

Temporal response The time dispersion of Cherenkov photons across a camera image depends on the energy of the primary gamma ray. At low energies, the dispersion is only few nanoseconds. Matched short signal integration windows are used to minimise the noise. The photo-sensor must not significantly lengthen the time structure of a Cherenkov light pulse. It is desirable to determine the pulse arrival times with sub-nanosecond precision for sufficiently large light pulses.

Lifetime Sensors will detect photons from the night-sky background at a typical rate of about $100-200 \mathrm{MHz}$ for the telescopes with large collection areas (MST and LST). If operation is attempted when the moon is up, this rate can increase by an order of magnitude. Sensors should have a lifetime of 10 years for an annual exposure of up to $\sim 2,000 \mathrm{~h}$. This can be achieved using PMTs with only 6-8 dynodes, operated at a gain of 30,000-50,000, followed by a fast AC-coupled preamplifier.

Rate of spurious signals Spurious signals from photo-detectors can result in an increase of trigger rates and a degradation of trigger thresholds. This is a particular issue for photomultiplier sensors where residual gas atoms in the tubes are ionised by impinging electrons. The resulting afterpulses, produced by positively charged heavy ions bombarding the photo-cathode, may have large amplitude and long delays relative to the primary electron. Photomultipliers should be selected with an afterpulse probability below $\left(\sim 10^{-4}-10^{-5}\right)$.

Operational characteristics To ensure efficient and reliable operation of the systems, sensors should show good short- and medium-term stability, and only gradual ageing, if any. Sensors should be able to survive high illumination levels. 
Cross-talk Although cross-talk for photomultipliers is very low, it may be an issue for alternative sensor solutions such as silicon photomultipliers or multi-anode photomultipliers. Crosstalk between adjacent pixels must be kept $\leq 1 \%$.

Cost and manufacturing considerations In total, the CTA consortium is intending to use $\sim 10^{5}$ sensor channels. Thus, the photo-detectors comprise a major fraction of the total capital cost of the project and any innovations which allow their cost to be reduced should be carefully considered. One important criterion is that the manufacturer/supplier must be able to provide the necessary number of sensors to the required specification with an acceptable and reliably known lead time.

Candidate photo-detectors The baseline photo-detector for CTA is the PMT. However, there may be alternative solutions that reach maturity on approximately the right timescale for CTA construction. Modular cameras for the LST, MST and SST are therefore desirable to allow the exchange of photodetectors without major alterations to the trigger and readout electronics chain. In the case of a secondary optics design of the MST or SST, conventional PMTs are not available in the appropriate physical size, and therefore the choice of a secondary optics telescope design would depend heavily on the availability of alternative photo-detectors, such as those presented here.

Baseline solution-photomultipliers The spectral sensitivity of conventional PMTs, see Fig. 43, with their falling sensitivity at large wavelengths, provides a reasonably good match to the spectrum of Cherenkov light on the ground. The baseline solution for CTA is to use PMTs with enhanced quantum efficiency compared to those currently used in H.E.S.S., for example. Such tubes are becoming commercially available and offer $\sim 50 \%$ advantage in photon detection efficiency over conventional PMTs.

Fig. 43 Spectral response of several types of super bi-alkali PMTs from Hamamatsu (green, red and black) and Electron Tubes Enterprises (yellow and blue), compared to the spectrum of Cherenkov light produced by vertical $100 \mathrm{GeV}$ gamma rays on the ground (grey, dashed), convoluted with the standard atmospheric transmission for the observation height of 2,200 $\mathrm{m}$ a.s.1.. The numbers in the inset give the convolution of the QE curve of a given PMT with the dashed line

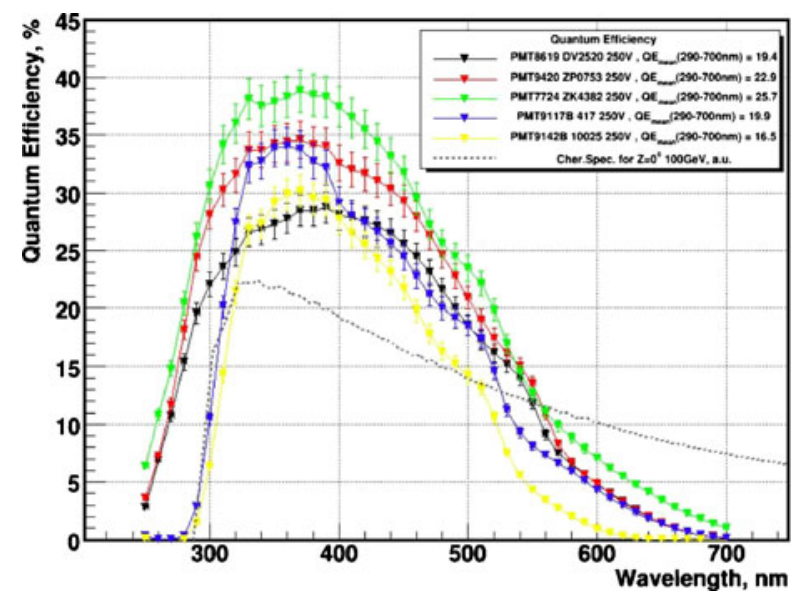


Silicon photomultipliers (SiPMs) (known also as MPPCs, GAPDs and Microchannel APDs) are novel light sensors that are rapidly reaching maturity. The more recent SiPMs consist of single pixels which contain several hundred to thousands cells, coupled to a single output, Each cell is operated in Geiger mode. An arriving photon can trigger the cell, after which that cells suffers significant deadtime, but leaves the surrounding cells ready to collect other arriving photons. The photon-counting dynamic range is comparable to the number of cells. Silicon photo-sensors could provide higher photon detection efficiencies than the latest PMTs at lower cost and without the requirement for high-voltage. However, silicon sensors typically require cooling to reduce the dark count to a manageable level and also suffer from optical cross-talk and are not as well matched to the Cherenkov light spectrum as PMTs. They therefore require further improvement and commercialisation. However, depending on the time scale and cost of such a development, SiPMs could be considered as a candidate sensor for replacing the PMTs or, alternatively, as an upgrade path for all telescope sizes. They are of particular interest for the SST secondary optics option, where their physical size is better suited to the plate scale of the telescope.

Multi-anode photomultipliers MAPMTs provide multiple pixels in a compact package, with properties similar to monolithic PMTs. Such devices offer individual pixel sizes of the order of $6 \mathrm{~mm}$, suitable for secondary optics schemes. Enhanced quantum efficiency versions with up to 64 channels are now available. The suitability of MAPMTs must be assessed, and properties such as the uniformity, cross-talk, dynamic range and detection efficiency are currently under investigation.

\section{Associated systems}

Light-collecting Winston cones Winston cones placed in front of any sensor could reduce the required sensor size by a factor of 3-4 (see Fig. 44). However, compression is limited by Liouville's theorem, which states that the phasespace volume of an ensemble of photons is conserved. Lightcones can minimise the dead space between pixels and reduce the amount of stray light from the night sky impinging on the sensors at large incidence angles. Figure 44 illustrates the typical angular response of a light funnel. Current lightcones have a net transmission of about $80 \%$. Improved cones may allow increased performance at modest cost.

Plexiglas input window To avoid the deposition of dust on the photodetectors and lightcones (if used), a Plexiglas window could be utilised to seal the camera. The transmission losses of $\sim 8 \%$ for a $3 \mathrm{~mm}$ thick GS 2458 window may be considered as well-justified because of the absence of deterioration of the light throughput on long time scales. The use of a sealed camera and plexiglas window must be investigated for each telescope size. For the LSTs and MSTs, sealing the camera does not significantly increase its total cost. For 

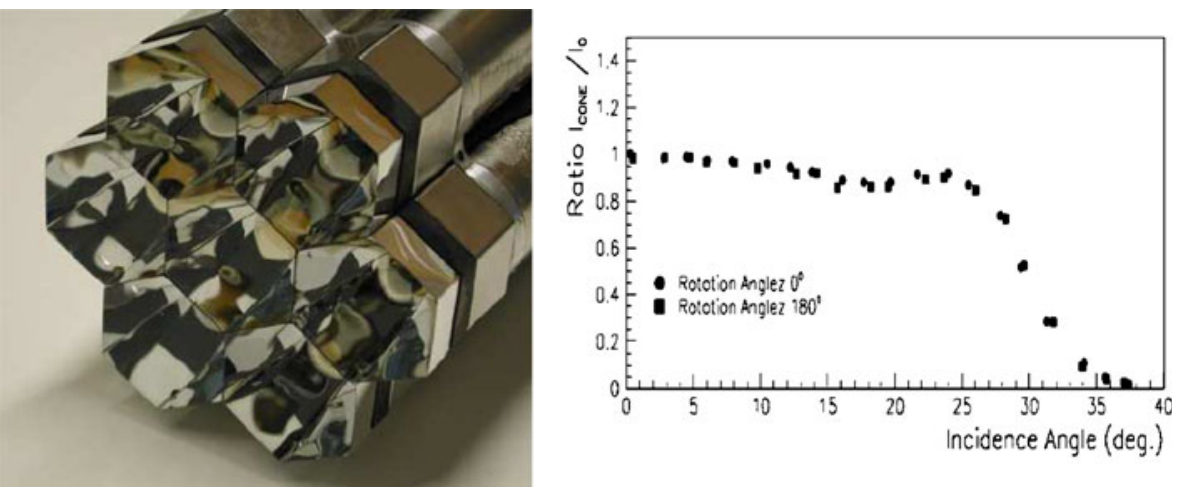

Fig. 44 Left PMT pixel cluster with light funnels. Right Angular response of a typical light funnel, normalised to the on-axis response

the SST, a sealed system may represent a significant proportion of the cost for the camera, but give advantages in maintenance and long-term performance.

$H V$ supply PMTs and MAPMTs need to be provided with a stable and adjustable high voltage supply. The first dynodes are often supplied through a passive divider chain, the last using an active divider to provide more power, improving the dynamic range and allowing stabilisation. The HV system also needs to provide a current-limiter or over-current trip circuit for protection in case of excessive illumination of the PMTs, due to bright stars, moon shine or, even worse, daylight. Several options are under study for CTA: (a) CockcroftWalton type, (b) transistor-based active divider type and (c) one central power supply providing individually attenuated voltages to different channels.

\subsubsection{Electronics}

Signal recording electronics Air-shower induced photo-sensor signals have a pulse width of a few ns, superimposed on a random night sky background with typical rates of some $10 \mathrm{MHz}$ to more than $100 \mathrm{MHz}$, depending on mirror size and pixel size (which is therefore different for the LST, MST and SST). Optimum capture of air-shower signals implies high bandwidth and short integration times. Ideally, the dynamic range and noise should be such that single photoelectron signals are resolved, and signals of a few thousand photoelectrons are captured without truncation. The recording electronics must delay or store the signals whilst a trigger is generated, indicating that the event is to be captured and read out. The generation of a trigger signal could take from 0.1 to a few $\mu$ s within a single telescope, depending on the complexity of the trigger scheme, and $\geq 10 \mu$ s if trigger signals between several telescopes are combined.

Advances in signal recording and processing provide the possibility of recording a range of signal parameters, from the integrated charge, to the full 
pulse shape over a fixed time window. Whilst it is not yet clear that the full pulse shape is needed, it is desirable to record at least a few parameters of the pulse shape rather than just the integrated charge. In this way, absolute timing information would be available, allowing improved background rejection and adaptive integration windows. Increasing the bandwidth of the signal recording system will allow improved timing and shorter integration gates, resulting in reduced levels of night sky background under the signal. However, as the bandwidth of the system is increased, so is the cost. Whilst such an approach may be justified for the LST, where night sky background is high, the Cherenkov pulses are very fast and the number of telescopes is low, this is not necessarily the case for the SST, where the night sky background is low, the Cherenkov pulses are not as fast and any cost savings could be used to build more telescopes. The bandwidth of the electronics chain for a given telescope size should be motivated by examining its consequences for the array sensitivity and energy threshold through Monte Carlo simulations. Currently, there is no clear answer as to the optimum choice for any telescope size, and the signal sampling frequencies under discussion range from a few $100 \mathrm{MSample} / \mathrm{s}$ to $\sim 2$ GSample/s.

Two techniques for signal recording and processing are in use in existing IACT arrays, These are based around Flash Analogue-to-Digital Converters (FADCs) and analogue sampling memories, and form the basis for the CTA development:

Flash analogue-to-digital converters FADCs digitise the photon-sensor signals at rates of a few $100 \mathrm{MSample} / \mathrm{s}$ to a few GSample/s, writing the output into a digital ring buffer, often realised as a very large scale integration gate array which also provides control logic and digital readout. The modest cost of digital buffers allows large trigger latency; delays of tens of microseconds can be realised. However, the dynamic range of FADCs is limited and typically no more than $8-10$ bits are available, requiring either parallel conversion with different gains or dynamic gain switching, as used in the $500 \mathrm{MSample/s,} \mathrm{8-bit}$ VERITAS FADC system [106]. The rather high cost of the fastest FADCs has led to the development of systems in which several channels are timemultiplexed onto one ADC, as used in the MAGIC 2 GSample/s, 10-bit FADC system. In principle, FADC based recording systems allow the use of a purely digital trigger, acting on the digitised data in the ring buffer, to select air shower events. Such a system is sketched in Fig. 45. None of the systems implemented so far uses this approach. Instead, parallel analogue trigger circuitry is used, adding not insignificant complexity to the electronics layout. The steadily increasing power of VLSI gate arrays may soon make digital trigger processors an attractive and feasible option.

As well as being expensive, FADCs suitable for IACTs are traditionally also bulky and power hungry, negating the possibility of integrating the readout electronics into the camera and requiring the transmission of analogue signals over many tens of meters to a counting house. However, the recent development of low-power, low-cost FADCs in recent years imply this situation 
Fig. 45 FADC based recording systems with purely digital trigger acting on the digitised data

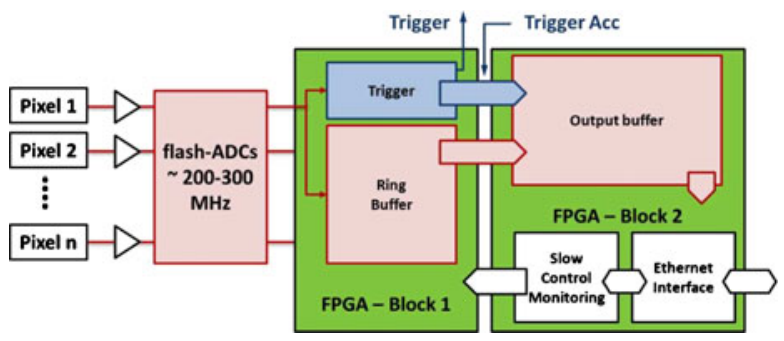

may be changing, at least for modest-speed FADCs. In response to this, a $250 \mathrm{MSample/s}$ system, named FlashCam, is under development for CTA. Monte Carlo simulations have shown that, at least for the MST and SST, $250 \mathrm{MSample} / \mathrm{s}$ is a sufficiently fast sampling rate to allow correct pulse shape reconstruction. Hardware prototyping is under way to confirm this simulated result. The sensitivity of the complete array with such a readout system must still be assessed.

Analogue sampling memories Analogue sampling memories consist of banks of switched capacitors which are used in turn to record the signal shape. The maximum recording depth is given by the sampling time multiplied by the number of storage capacitors, which ranges from 128 to a few 1,000, implying at most a few microseconds of trigger latency. Trigger signals are derived using additional analogue trigger circuits.

Current ASIC implementations stop the recording of signals after a camera trigger and initiate the digitisation of the charge stored on a selected range of capacitors, thereby introducing front-end deadtime of few microseconds. The signal is then converted to a digital format using an ADC and can be stored in a local Field-Programmable Gate Array (FPGA) before transfer (see Fig. 46). The ADC is typically used to digitise the pulse integrated over a time window, and therefore can have a sampling frequency an order of magnitude lower than those considered in the FADC readout scheme. Additional information, such as the pulse width and arrival time, can also be stored, which is highly desirable. A First-in, First-out (FIFO) memory between the digital conversion

Fig. 46 Analogue memory based recording systems. The analogue trigger is formed in parallel to the data shaping and buffering

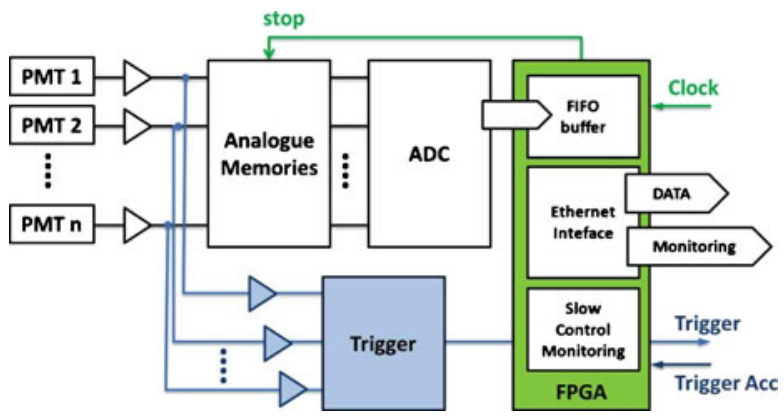


Table 4 Characteristics of switched-capacitor signal-recording ASICs

\begin{tabular}{llllll}
\hline & ARS & SAM & NECTAR & DRS4 & TARGET \\
\hline GSamples/s & 1 & $1-3.2$ & $1-3.2$ & $0.2-6$ & 1 \\
Channels/chip & 5 & 2 & TBD & 8 & 16 \\
Samples/channel & 128 & 256 & $>1,024$ & 1,024 & 16,000 \\
Analogue bandwidth $(\mathrm{MHz})$ & 80 & $>400$ & 300 & 950 & 380 \\
Dynamic range (bits) & $9-10$ & 12 & $\geq 12$ & $11-12$ & $9-10$ \\
Integrated trigger disc. & No & No & No & No & Yes \\
Integrated ADC & No & No & Yes & No & Yes \\
Integrated digital control logic & (PLL) & Yes & Yes & (PLL) & Yes \\
Typ. readout latency $(\mu \mathrm{s})$ & 60 & $<2$ & $<2$ & $<3$ & $<3$ \\
Power cons. $(\mathrm{mW})$ & 500 & 300 & $150-300$ & 65 & 10 \\
Status/use & HESS I & HESS II & Design & MAGIC II & Design \\
\hline
\end{tabular}

and the FPGA can be used to smooth the distribution of arrival times of events to reduce fluctuations in the data acquisition rate.

The dynamic range of analogue samplers is up to 12 bits. As with FADCbased systems, parallel channels with different gains or non-linear input stages can be employed to record a larger dynamic range of Cherenkov signals. Examples of such systems include the HESS I readout system, which is based on the ARS ASIC [107], the HESS II readout, based on the further developed Swift Analogue Memory (SAM) ASIC with significantly reduced readout deadtime [108], and the MAGIC II readout system, using the Domino Ring Sampler (DRS) ASIC [109]. Several analogue sampling based schemes are under development for CTA, including a project based on the next-generation version of the SAM chip, termed NECTAr, a DRS4 based project called Dragon and a project based on the Target ASIC originally intended for AGIS. The main parameters of some of these ASICs are summarised in Table 4.

While FADC systems may ultimately offer somewhat superior performance, analogue samplers could allow lower cost, in particular if much of the auxiliary circuitry surrounding and supporting the sampler ASIC, such as pixel trigger circuits, ADCs, digital buffer and readout controllers can be integrated into a single multi-channel ASIC (Fig. 47). This is analogous to the readout for silicon strip sensors, where single readout ASICs typically accommodate 128 channels and where the cost per channel is at the level of a few $€$.

Fig. 47 High-level integrated analogue sampling ASIC. The single ASIC amplifies, stores, and digitises the analogue signal, and buffers the digital data before sending them to the central camera recording system

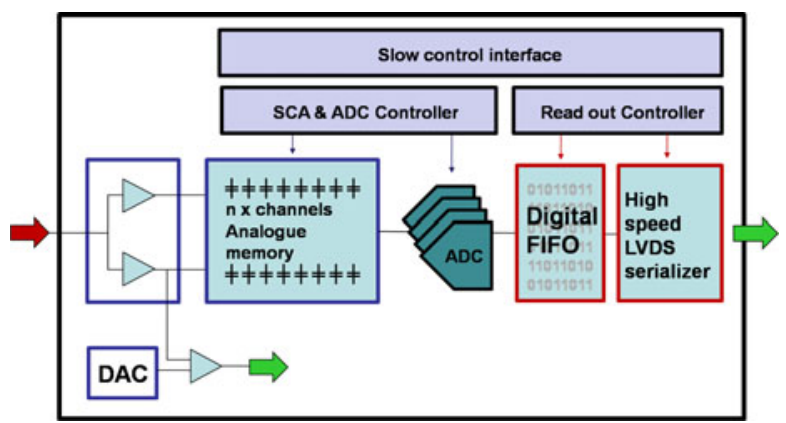


At the current stage of CTA electronics design, analogue samplers and FADCs will be pursued in parallel. Existing ASICs such as the SAM or DSR4 are probably adequate for use in CTA. MC simulations should help to decide if dual-gain channels are needed, which would imply significantly increasing electronics cost. A specific development effort is also aimed at producing nonlinear input stages providing signal compression.

Readout electronics Readout of digitised data has so far either relied on custom-built bus systems to collect data from electronics units covering the camera focal plane (such as the "drawers" of the H.E.S.S. telescopes), or has located the digitisation electronics in commercial VME or PCI crate systems. As a flexible and cost-effective alternative, the use of commercial Ethernet systems has recently been explored [110], using normal switches to buffer data sent via a low-level Ethernet protocol (Fig. 48). A low-cost front-end gate array emulates the Ethernet interface. Data transfer is asynchronous, with buffering in the front-end gate array, eliminating a source of deadtime. To enable synchronisation, events are tagged at the front end with an event marker. In tests with 20 sender nodes, transmitting via a switch to a receiver PC, loss-free transmission of more than $10^{10}$ packets with a data rate of more than $80 \mathrm{MByte} / \mathrm{s}$ was achieved. Current up-to-date servers can operate with $2 \times 4$ GBit interfaces and cope with the resulting data flow. It is therefore expected that loss-free transmission of the front-end data, even of a 2,000 pixel camera operating at data rates of $600 \mathrm{MByte} / \mathrm{s}$, should not be a problem. Nevertheless, various forms of zero suppression could be implemented in the front end, reducing data rates by up to an order of magnitude. Since the Ethernet system operates in full-duplex mode, it can also be used for the control and parameterisation of the front-end components, such as HV supplies, and to set parameters for triggering, digitisation etc. It would not be necessary to design a separate command bus, as employed in most current cameras.

Fig. 48 Possible scheme for an Ethernet-based front-end to back-end readout. A group of pixels with their ADCs is controlled by a dedicated FPGA. The same FPGA can be used to buffer the data and to transmit them through a dedicated Ethernet network to a camera computer (PC Server), which buffers the data in its RAM and preprocesses events before sending them to an event building farm

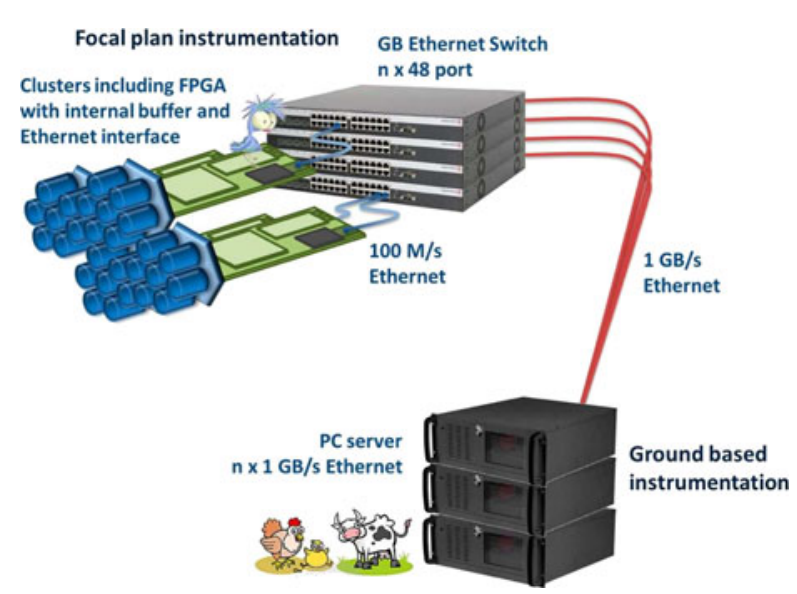




\subsubsection{Triggering}

Triggering the telescopes Arrays of Cherenkov telescopes typically employ multi-level trigger schemes to keep the rate of random triggers from the night sky background low. At the first level, signals from individual pixels are discriminated above a threshold. These pixel-level signals are input to a second level, topological trigger. The topological trigger is used to identify concentrations of Cherenkov signals in local regions of the camera, via patten recognition or a sum of first-level triggers, to form a telescope-level trigger. A third, array-level trigger, is formed by combining trigger information from several telescopes.

The trigger chain within a telescope may follow a digital, or analogue path. In H.E.S.S., Magic and VERITAS, analogue schemes are used, but for CTA several approaches for both options are under investigation. A digital scheme would require the continuous digitisation (with one or more bits) of the signal coming from the PMTs. Components that look for coincidences from digitised signals with a predefined timing are commercially available. In a digital scheme, the trigger is very flexible and almost any algorithm can be implemented, even a posteriori. Trigger algorithms and parameter settings for each camera can easily be adapted for each telescope type, array configuration and for the physics programme (e.g. energy range). Both sector and topological trigger concepts can be implemented in a digital trigger system. The information provided by a digital trigger is essentially "screenshots" of the camera every given time slice. Even if only one or a few bits are used to encode the trigger information, it may be worthwhile to add this information to the data stream. In the extreme case of a digital trigger with sufficient resolution, only the digital stream could be used, as is proposed for the FlashCam development. On the other hand, the digitisation frequencies available at reasonable cost may yield worse rejection of random triggers from the night sky background compared to an analogue approach.

The telescope trigger is traditionally formed by looking for a number of pixels above threshold, or a number of neighbouring pixels above threshold, within the camera. This is typically implemented by dividing the camera up into sectors, which must overlap to provide a uniform trigger efficiency across the camera. By requiring several pixels to trigger at once, random fluctuations due to the night sky background and PMT afterpulses are greatly reduced. Alternative schemes are also under investigation. These include a sum trigger, which can lead to a significant reduction of the trigger threshold [111]. In the sum trigger, the analogue or digital sum of all pixels in a cluster is formed and a threshold is set to initiate a trigger. It is necessary to clip pixel signals before summing to prevent large afterpulses triggering a cluster (see Fig. 49). All these approaches can be implemented in both an analogue or a digital path.

The size of camera sectors and their overlaps have implications for the threshold and detection efficiency. Given the different goals, they may differ for the LST, MST and SST. Schemes for all telescope sizes are under investigation using Monte Carlo simulations. The shape and dimensions of 
Fig. 49 Trigger rate (in $\mathrm{Hz}$ ) against the discriminator threshold (in photo electrons). NSB dominates at low thresholds. Without clipping afterpulses largely dominate the rate (blue), while they are effectively eliminated by clipping (red). The black points beyond 25 photo-electrons are due to cosmic showers

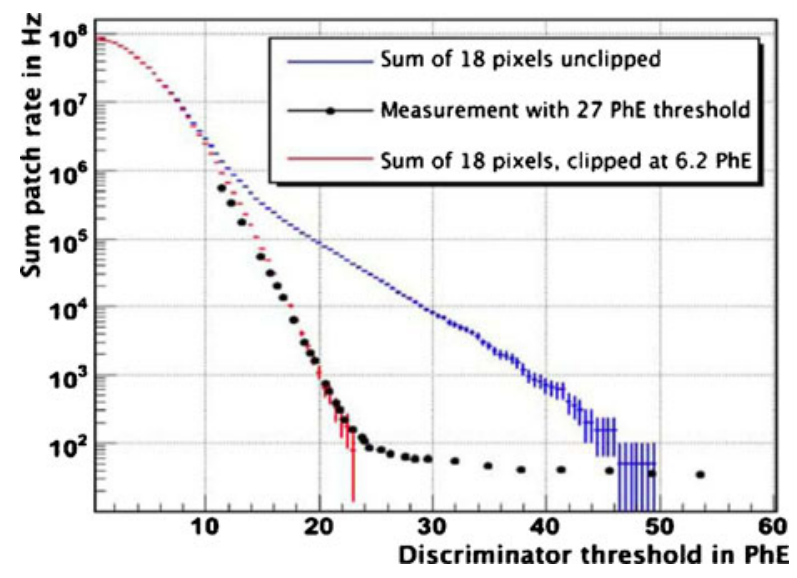

the mechanical clusters put limitations on possible camera sectors and their overlap, but these are only of second order. The default reaction to a trigger is the read out of the entire camera. Also an autonomous-cluster trigger is under study, however. This allows sections of the camera to form trigger decisions independently and these are read out autonomously. In the highenergy range, observations at low elevation produce images that propagate in time through the camera. The propagation time can be much higher than the usual integration window of the Cherenkov signal acquisition. The autonomous readout would allow the recording of the time slot when the signal is in each section of the camera, following the propagation of the image through the camera. For low energy showers, the shower image covers a small region of the camera and it can be useful to read out only a part of the focal plane to save bandwidth on the network and to lower the deadtime of the system.

The earlier a trigger system enters a purely digital level, the more easily and reliably it can be simulated. Schemes which rely on the addition of very fast analogue or digital signals are potentially more powerful, but could be sensitive to details of the pulse shapes and to the transition-time dispersion between different PMTs, requiring a pre-selection of PMTs with similar transition times or the implementation of matched delays to compensate for intrinsic differences.

Note that no signal recording scheme rules out the use of a given triggering scheme, but the use of FADCs to record the signal would allow implementation of a digital trigger based on the already digitised signals, hence reducing the cost and complexity of the system.

Triggering the array Current array trigger schemes for systems of Cherenkov telescopes [72] provide asynchronous trigger decisions, delaying telescope trigger signals by an appropriate amount to compensate for the time differences when the Cherenkov light reaches the telescopes, and scanning trigger signals 
for pre-programmed patterns of telescope coincidences. The time to reach a trigger decision and to propagate it back to the telescopes is about $1 \mu \mathrm{s}$ or more. While FADC based readout systems can buffer signals for this period, analogue-sampling ASICs will usually not provide sufficient memory depth, and require the halting of waveform sampling after a telescope trigger, while awaiting a third-level telescope coincidence trigger. The resulting deadtime of a few $\mu$ s limits telescope (second-level) trigger rates to some $10 \mathrm{kHz}$, which does not represent a serious limitation. The latest analogue-sampling ASICs allow digitisation of stored signals on time scales of 2-3 $\mu$ s (see Table 4), comparable to the array trigger latency. In this case, a new option for the triggering of the array becomes possible: pixel signals are readout and digitised after each telescope trigger, and are stored in digital memory, tagged with an event number. Given that data are buffered and that buffers can easily be made large, restrictions on array trigger latency are greatly relaxed (with GByte memory, about $1 \mathrm{~s}$ of data can be buffered) and one can implement a softwarebased asynchronous trigger. With each local trigger, an absolute timestamp is captured for the event with an accuracy of the order of 1 ns and transmitted to the camera CPU. This computer collects the time stamps and possibly additional trigger information for each event, e.g. pixel trigger patterns, and transmits them every 10-100 ms via standard Ethernet using TCP/IP to a dedicated central trigger computer. The central computer receives all time stamps from all telescopes and uses this information to test for time coincidences of the events and to derive the telescope system trigger. In addition, the time and trigger information can be used to obtain a first estimate of the core position and shower direction. Following the central trigger decision, the central trigger CPU sends the information to the corresponding telescopes about which of the buffered events are to be rejected and which fulfil the system trigger condition and should be pre-processed in the camera CPU and transmitted for further stereoscopic processing. Assuming a local trigger rate of $10 \mathrm{kHz}$ and that about 100 Byte of trigger information are generated from each telescope, the central trigger computer needs to handle up to $100 \mathrm{MByte} / \mathrm{s}$ in a 100 telescope system, which can be readily be done with today's technology. In such a trigger scheme, the central trigger decision is software-based, but the "hard" timing from the camera trigger decision is used. It is therefore scalable, fully flexible and all types of sub-systems can be served in parallel. At the same time it uses the shortest possible coincidence gates and provides an optimum suppression of accidental coincidences.

\subsubsection{Camera integration}

Signal transmission from the photo-sensors to the recording electronics represents a critical design issue if the electronics is located far from the photo-sensors. Conventional cables limit bandwidth, are bulky and difficult to route across telescope bearings, and are costly. MAGIC uses optical signal transmission, circumventing the first two problems, at considerable expense. H.E.S.S. avoids signal transmission altogether by combining 16 photo-sensors 
and their associated electronics in "drawers", requiring only power and Ethernet connection to the camera [112], but limiting flexibility as regards upgrades of individual components.

At least for the SST and MST, which are produced in significant quantity and where costs of the electronics is a decisive factor, the most effective solution seems to be to combine photo-sensors and electronics in the camera body. The design should allow easy swapping of the camera for a spare unit, allowing convenient maintenance and repair of faulty cameras at a central facility. However, over the expected lifetime of CTA, upgrades at least of the photo-sensors are likely. The same may be true for the trigger and data recording systems, where novel networking components may allow transmission of significantly larger amounts of digital data than is currently possible. A viable option could therefore be, rather than combining photosensors and electronics in a single mechanical unit, to build a photo-sensor plane with short connections to electronics units, which in turn feed a trigger system via a flexible interface (Fig. 51). For ease of mechanical assembly, both photo-sensors and electronics will be packaged into multi-channel units.

Dual-mirror solutions, such as the Schwarzschild-Couder telescopes, require much smaller cameras and can therefore utilise cheap multi-anode photo-sensors. Figure 50 shows a possible solution considered for AGIS, using 64-pixel multi-anode PMTs [113].

Mechanical packaging of the entire camera and sealing against the environment is crucial for stable performance (Fig. 51). In its daytime configuration with closed camera lid, the camera body should be reasonably waterproof. Dust penetrating the camera and deposited on connectors and on optical components is a serious issue. To protect the photo-sensors and the lightcollecting funnels and allow for easy cleaning, an optical entrance window
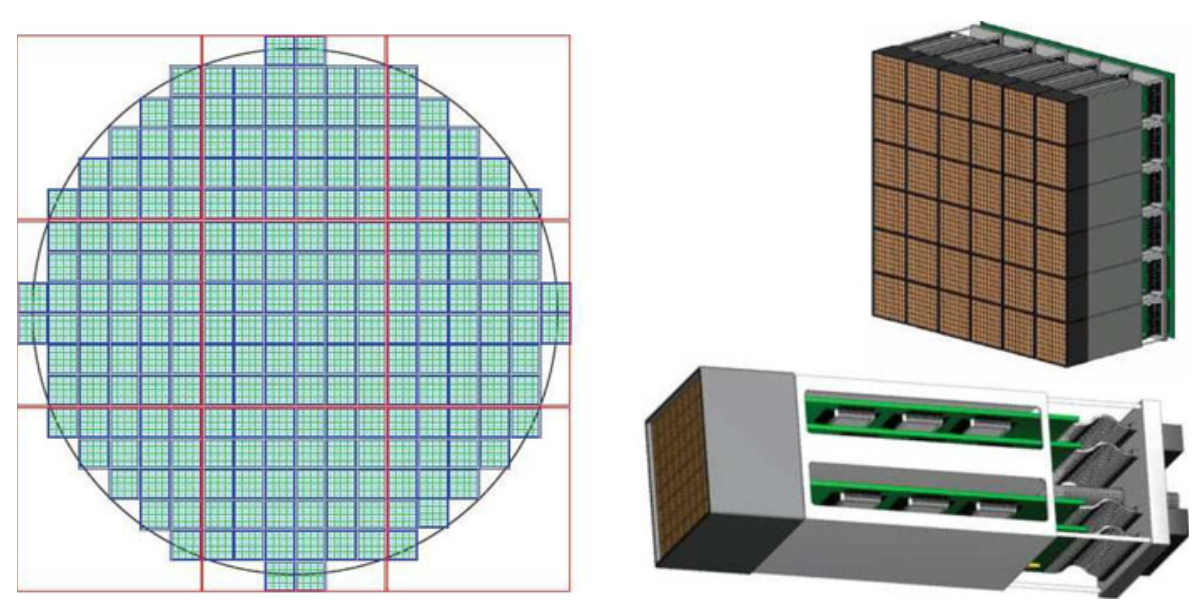

Fig. 50 Instrumentation of a $50 \mathrm{~cm}$ diameter camera for a dual mirror telescope from 64-pixel multi-anode PMTs. One pixel is about $6 \times 6 \mathrm{~mm}^{2}$ 
Fig. 51 Concept for packaging of the electronic contained in the camera



made of near-UV transparent material is desirable, even if this induces a modest light loss due to reflection. While larger-scale integration should reduce power consumption compared to current systems, a camera will nevertheless consume kilowatts of power and must be cooled. Air cooling requires high-quality filtering of the airflow into the camera. Closed-circuit cooling systems, involving internal circulation of a cooling medium and appropriate heat exchangers improve long-term reliability, but add cost and weight.

\subsection{Calibration and atmospheric monitoring}

The higher sensitivity of CTA means good gamma-ray statistics for many sources. Therefore, the instrument's systematic uncertainties may limit the accuracy of the measurements. The atmosphere is an integral part of an IACT and so monitoring and correcting for atmospheric inhomogeneity must be addressed in addition to the detailed calibration and monitoring of the response and characteristics of the telescopes. Work is ongoing to address both issues, as well as their interplay, with the goal of characterising the systematic uncertainties to an unprecedented level.

Already teams of world-experts have gathered to develop state-of-the-art instrumentation for atmospheric monitoring and the associated science for CTA. These teams are actively participating in the corresponding CTA work package (ATAC).

\subsubsection{Telescope calibration}

The calibration of the CTA telescopes has two distinct aspects. Firstly, the absolute gain of the system must be determined. Secondly, the pointing accuracy of the telescope must be measured. The necessity of the precise measurement of the gain of each electronic channel for CTA requires the development of a single and reliable calibration device which can measure the flatfielding coefficients and the ratio between a single photoelectron and the number of digital counts recorded. This development will add to existing experience in building calibration devices, for example the H.E.S.S. II flatfielding system as 
Fig. 52 Layout of the H.E.S.S. II flatfielding and single photoelectron device. For a large array of telescopes, it is likely that the laser will be replaced by LEDs and that the mechanical filter wheel will be replaced by an electronic system

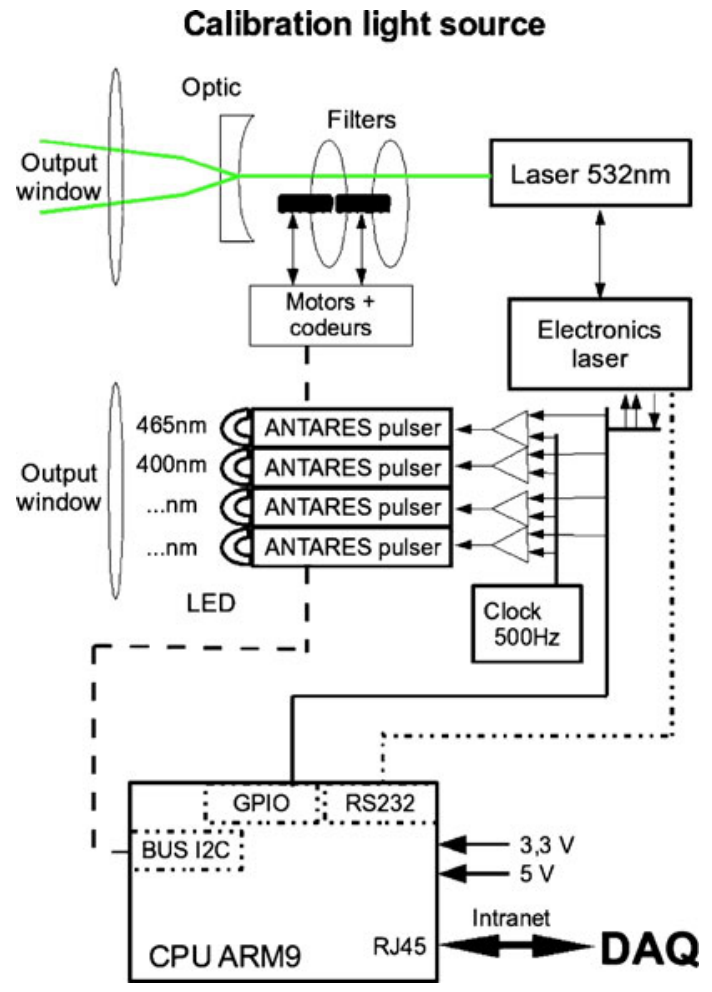

shown in Fig. 52. Overall, absolute calibration is achieved by reconstructing the rings generated by local muons. A special pre-scaled single-telescope trigger could be implemented to enhance the rate at which these are recorded.

In the development of the calibration apparatus, many challenges must be addressed. The first is the difficulty of uniformly illuminating large, wide fieldof-view cameras. This problem is twofold: firstly, diffusers must be able to present a uniform signal to the edge of the field of view; secondly, the pixels across the field of view must uniformly accept the diffused signal on their photo-cathodes. This second aspect can be difficult to achieve when reflective lightcones on the camera edge have a different acceptance to those in the centre to a close-by, centrally diffused light source. The use of different colour light sources would allow the quantification of any differences and/or changes in the quantum efficiency of pixels. An additional challenge concerns the measurement of the single photo-electron response. Current telescope systems measure this either in-situ with a low light background level, or indirectly using photon statistics [114]. A comparison of these two methods allows the study of their associated systematic errors and the choice for the best system for CTA.

The requirements of the telescope pointing measurement are somewhat simpler, but vitally important. Here, a system of two CCD cameras mounted on each telescope is envisaged. The first measures the position of the night- 
sky relative to the telescope dish and the second the position of the telescope camera relative to the dish. In combination, the system allows the astronomical pointing of the telescope to be assessed accurately.

\subsubsection{Atmospheric monitoring}

The calibration of the CTA telescopes is one critical calibration and monitoring task, a second is the monitoring of the atmosphere which forms part of the detector. This is where the particle shower is initiated by the incident gamma-ray and the medium through which the Cherenkov photons must travel. The estimation of the energy of an individual gamma-ray is based on the calorimetric energy deposited in the atmosphere, which in turn is measured via Cherenkov photon emission. Therefore, any change in atmospheric quality can affect the signal detected. To investigate this effect, a set of benchmark simulations of a 97-telescope array design were initiated to test the performance of an array of imaging Cherenkov telescopes under the presence of varying atmospheric conditions. Simulations were produced for a clear atmosphere and an atmosphere with a significant layer of low-level dust, as derived from measurements taken with a $355 \mathrm{~nm}$ single-scattering Lidar deployed on the Namibian Highlands. These show that, if unaccounted for, the changing atmospheric quality produces a significant shift in the reconstructed gammaray spectrum. This can be seen in Fig. 53.

Many current Cherenkov telescope arrays have in-situ single-scattering Lidars. This type of Lidar possesses a strong and variable systematic error in the derived transmission, up to approximately 50-60\% [115]. After discussions with members of the Pierre Auger Observatory (PAO) and other atmospheric scientists, CTA has decided to adopt the Raman Lidar technique as the tool of choice for accurately probing atmospheric quality. Below and around shower maximum, it is believed that this technique will reduce the systematic error in derived transmission to approximately 5\% [115]. Therefore, Raman Lidars are currently under development, which will be installed at the sites of some existing Cherenkov telescopes in order to test their efficacy in ground-based gamma-ray analysis. If successful, these atmospheric monitoring systems will allow CTA to significantly reduce the systematic error in energy measurements and derived source fluxes.

\subsection{Quality assurance}

Since the design study phase, the CTA project has included a work package named "Quality Assurance and Risk assessment". The objective of this WP is to implement a uniform approach to risk analysis in the design, commissioning and operation of the telescopes and of the facility, and for quality assurance of the telescope components and of the assembly procedures.

"Risks" are any features that can be a threat to the success of the project. They can have negative effects on the cost, schedule and technical performance of CTA. The aim of project risk management is to identify, assess, reduce, 


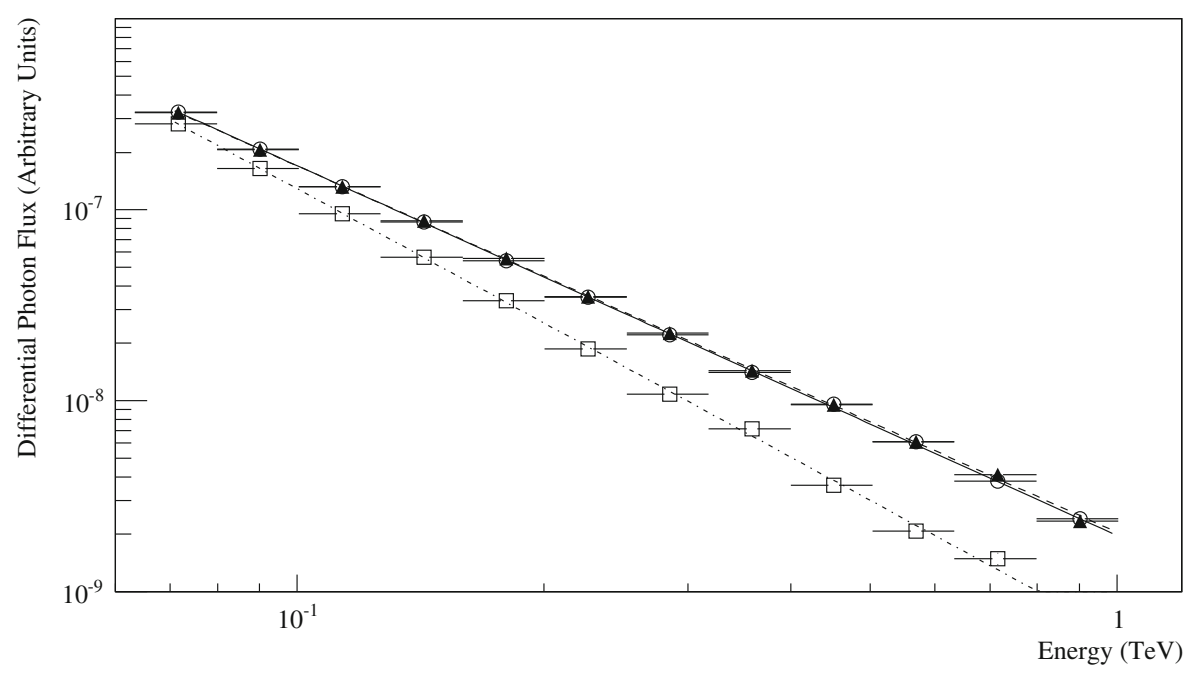

Fig. 53 Recovering spectral information for non-ideal observing conditions. From a full simulation database a randomly sampled spectrum of $10^{5}$ events with spectral slope of $\mathrm{E}^{-2.3}$ is drawn. These events are then reconstructed using simulation-based look-up tables which give the reconstructed energy as a function of the camera image brightness and the reconstructed distance to the shower. For different atmospheric conditions (described in Table 5), a reconstructed spectrum is derived. The open circles show the reconstructed differential spectrum for case 1 , the open squares for case 2 and the closed triangles for case 3. By incorporating Lidar data into the reconstruction (case 3 ) a corrected spectrum can be recovered with approximately the same normalisation and slope as for a clear night sky (case 1)

accept (where necessary), and control project risks in a systematic and costeffective manner, taking into account technical and programming constraints.

Quality Assurance ensures a satisfactory level of quality for all steps of the design study. This level of quality is guaranteed by correct implementation of the pre-defined quality criteria and the participation of all the project actors.

Including quality assurance and risk assessment from the very start of the design study phase will have a positive effect on the building schedule and cost of CTA.

The objective of the design study is to develop telescopes which will be produced in series during the building phase, so the study will be done in partnership with industry. Quality assurance and risk assessment will ensure that the project will have good traceability and a good control of risks from the outset.

Table 5 The combination of look-up tables (as derived from simulations) and simulated spectra produced to derive the effect of the atmosphere on reconstructed spectra illustrated in Fig. 53

\begin{tabular}{lll}
\hline Case & Simulation derived from & Lookup derived from \\
\hline 1 & Clear database & Clear database \\
2 & Dusty database & Clear database \\
3 & Dusty database & Dusty database \\
\hline
\end{tabular}


This WP is managed by a coordinator who defines standards and quality methods for the project. To ensure the implementation of quality in the project laboratories, "Local Quality Correspondents" (LQCs) will be identified and trained. These people will dedicate part of their time to quality issues, proportional to their laboratory participation in the overall project.

The main tasks of the WP participants are:

- To define the quality insurance organisation (the roles of the participants)

- To ensure that quality control and risk analysis procedures are defined and applied uniformly across the project to ensure high quality and reliability of hardware and software

- To ensure that the risk analysis, including dependability (reliability, availability, maintenance and safety) is defined based on the technical configuration proposed

- To ensure support and expertise to implement the quality system and associated tools across the project

- To verify the coherence of the procedures and protocols in order to approve them for subsequent release and use

- To verify the application of the quality procedures across the project

- To identify and reduce technical and management risks

Quality assurance and risk assessment concern the whole project. Thus, the members of "Quality assurance and risk assessment" will have active links to all work packages, to the project management and to all laboratories involved in building parts of CTA.

\section{CTA site selection}

Selection of sites for CTA is obviously crucial for achieving optimum performance and science output. Criteria for site selection include, among others, geographical conditions, observational and environmental conditions and questions of logistics, accessibility, availability, stability of the host region, and local support:

Geographical conditions For best sky coverage, the latitude of the sites should be around $30^{\circ}$ north and south, respectively. The sites have to provide a reasonably flat area of about $1 \mathrm{~km}^{2}$ (north) and at least $10 \mathrm{~km}^{2}$ (south). Optimum overall performance is obtained for site altitudes between about 1,500 and $4,000 \mathrm{~m}$. Even higher altitudes allow further reduction of the energy threshold [93] at the expense of performance at medium and high energies and might be considered for the northern array. Desirable is also a low component of geomagnetic field parallel to the surface, since such fields deflect air shower particles.

Observational conditions Obviously, the fraction of clear nights should be high. For good sites, this fraction is well above $60 \%$, reaching up to $80 \%$ for the very best sites. Artificial light pollution must be well below the 
natural level of night sky background, which excludes sites within some tens of $\mathrm{km}$ of major population centres. Atmospheric transparency should be good, implying dry locations with low amounts of aerosols and dust in the atmosphere.

Environmental conditions Environment and climate influences both the operational efficiency and the survival conditions of the instrument. Wind speeds above $10 \mathrm{~m} / \mathrm{s}$ may impact observations; peak wind speeds, which may range from below $100 \mathrm{~km} / \mathrm{h}$ to beyond $200 \mathrm{~km} / \mathrm{h}$ depending on the site, have a major impact on telescope structure and cost. Sand storms and hail represent a major danger for unprotected mirror surfaces. Snow and ice prevent observations and will influence instrument costs, e.g. by making heating systems necessary and requiring increased structural stability. Seismic activity will similarly increase requirements on telescope structures and buildings.

Infrastructure and logistics A well-developed infrastructure, e.g. as a result of already existing observatories, is an advantage. Connection to the power grid and high speed internet access are mandatory. There should be good access to the site, i.e. nearby airports for air travel to/from Europe and elsewhere, and local access roads. A major population centre with technical and commercial infrastructure within convenient travel distance is desirable.

Other criteria These include availability of the site for construction, guarantees for long-term operation and access, political stability of the host region, safety of personnel, both during travel and stay, and availability of local administrative, technical and funding support as well as possibilities for scientific cooperation with local groups.

For both the observational and the environmental conditions, a long-term (multi-year) data record is required to allow dependable decisions o be made. While archival remote-sensing data can provide some information, wellexplored sites with existing installations and good records are favoured. It is unlikely that any site is optimal in all respects, so the different criteria will have to be balanced against each other. Reliable and efficient operation of the observatory should be a key criterion.

Site evaluation includes a number of different approaches, at different stages of progress for a candidate site:

- Use of remote-sensing archival data and local archival data to evaluate observing conditions and environmental conditions.

- Site visits and information gathering by local collaborating groups on logistics aspects.

- Dedicated CTA measurements; since long-term measurements are excluded, this approach is useful only for those quantities where short campaigns can provide meaningful results, such as the determination of natural and artificial night-sky brightness. 


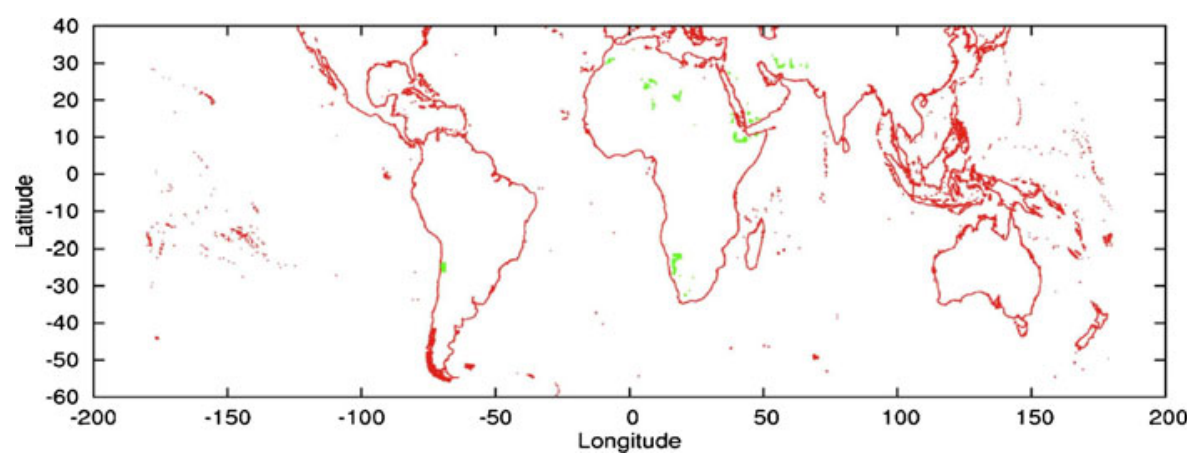

Fig. 54 Green areas indicate sites above 1,500 $\mathrm{m}$ a.s.1., which offer sufficiently flat areas, minimal artificial background light and an average cloud cover of $<40 \%$, selected on the basis of topological and satellite data

A first preselection can look for sufficiently large and flat areas above $1,500 \mathrm{~m}$ a.s.l. (based on a topological model of the Earth [116]), with the requirement that the artificial background light is minimal (as determined from satellite images [117]), and that average cloud coverage is less than $40 \%$ (as provided by the International Satellite Cloud Climatology Project, IS$\mathrm{CCP}$, based on the analysis of satellite data (http://isccp.giss.nasa.gov/products/ dataview.html)). The resulting map (Fig. 54) shows very few locations matching these basic criteria, among them the well-known sites in Chile and Namibia. However, while the ISCCP data has the advantage of covering the whole planet, the resolution is relatively coarse and sites with very local conditions (such as mountain tops) may deviate significantly from the "pixel" average. Also, daytime and nighttime cloud cover will usually be different. Only the latter is relevant for Cherenkov astronomy. For identification of potential observatory sites, special algorithms and high-resolution data have been provided by Erasmus [118] for the identification of potential observatory sites, but only for selected areas, such as the Chilean sites, the Indian site at Hanle or the Yanbajing site in Tibet. Similar searches are conducted using MODIS and ISCCP maps as well as the recently released ESO application FriOwl that provides access to an extensive database of information from the last 40 years (http://archive.eso.org/friowl-45/).

Based on these preliminary evaluations, potentially interesting sites have been selected at which detailed studies will be conducted in the coming months.

Northern site candidates are:

Canary Islands La Palma and Tenerife These are well-known and wellexplored observatory sites at about $26^{\circ} \mathrm{N}$, about 2,400 $\mathrm{m}$ a.s.l., with the Observatorio del Roque de los Muchachos on La Palma and the Observatorio del Teide on Tenerife. 
Hanle in India, in the Western Himalayas This high-altitude site $\left(33^{\circ} \mathrm{N}, 4,500 \mathrm{~m}\right.$ a.s.l.) hosts a small observatory and an array of Cherenkov instruments which is deployed by Indian groups.

San Pedro Martir, Baja California Well-established astronomical site that hosts already two observatories run by UNAM (Universidad Autonoma de Mexico). It is situated at about $31^{\circ} \mathrm{N}$, at 2,800 $\mathrm{m}$ a.s.1.

Southern site candidates are:

Khomas Highland of Namibia This is a well-known astronomical site, at 1,800 $\mathrm{m}$ a.s.l. and $23^{\circ} \mathrm{S}$, and is the home of the H.E.S.S. instrument. The region offers a range of suitable, large and flat areas.

Chilean sites Chile is home to some of the World's premier optical observatories. However, availability of sufficiently large cites near these locations is limited. A possible site is north of La Silla at $29^{\circ} \mathrm{S}$ and 2,400 m a.s.l. Another potential site is near Cerro Paranal, with even better observing conditions, but no sufficiently flat area in this region has been identified so far.

El Leoncito Reserve in Argentina This site is at $32^{\circ} \mathrm{S}$ and 2,600 $\mathrm{m}$ a.s.1. and hosts the El Leoncito Astronomical Observatory.

Puna Highland in Argentina The region offers some large sites at 3,700 m a.s.l. with sky quality equivalent to the best Chilean ones. These sites have good access to a railway line.

The final decision among otherwise identical sites may rely on considerations such as financial or in-kind contributions by the host regions. It is likely that an inter-governmental agreement will be required to assure long-term availability of the site, as well as guaranteed access and free transfer of data. At the same level, issues such as import taxes, value added tax and fees etc. should be addressed. Such agreements exist, for H.E.S.S., Auger and other observatories operated by international collaborations.

\section{Outlook}

The Cherenkov Telescope Array was conceived back in 2005, and was then promoted by members of the HESS and MAGIC collaborations. It was soon apparent that a gamma-ray observatory could be designed with existing technologies that was much more powerful than any of the existing facilities. An improvement of a factor of 10 in sensitivity around a $\mathrm{TeV}$ and an extension of the energy range from a few tens of GeV to $>100 \mathrm{TeV}$, well beyond the currently accessible range, was achievable with an array of a large number $(\approx 100)$ of differently sized telescopes.

With the results from current Cherenkov telescopes pouring in, it became obvious that with such an instrument a vast number of sources of very different types could be discovered and studied with unprecedented precision. Answers to long-standing questions in a number of science areas seemed possible. The 
extent and the diversity of the science case was, and is, stunning (see Section 3). CTA would truly be the first large open observatory for astronomy of the extreme universe beyond the $\mathrm{GeV}$ range.

Not surprisingly, many scientists were attracted to CTA and its science grew rapidly, as did the number of supporters who now form a large international collaboration which is investigating how best to realise the project. CTA has received consistently excellent reviews and high rankings in Science Roadmaps in Europe and across the world. CTA is an acknowledged ESFRI project, features high on the roadmaps of future projects of ApPEC, ASPERA and ASTRONET and has been well received by national funding agencies. The potential of CTA is well recognised outside Europe, with the USA, Japan, India, Brazil and Argentina, and other countries contributing significantly. The US Decadal Survey endorsed a strong US participation in CTA as one of the four most important ground based initiatives in the next ten years.

Since 2006, and specifically in a 4-year design study, it has been shown that CTA, with observatories in the northern and southern hemisphere, can be built to achieve its goal performance, at an investment cost in the range of $150 \mathrm{M} €$, which is a modest price for an installation of such scientific potential.

CTA has recently received substantial funding from the European Community, for preparing for construction and operation, and from national funding agencies, for development and prototyping. There is much excitement amongst all participants, and the wider science community, about the prospects that CTA will soon from design to reality.

In this report, an account of the main design work performed so far is presented, which constitutes a solid basis for the prototyping and construction phases that lie ahead. The Preparatory Phase (3 years) and the subsequent construction phase (2013-2018) will pose many challenges. But CTA is a wellorganised international collaboration of 25 countries and $>600$ scientists with extensive expertise in all relevant areas. Its members are eager and ready to tackle the problems that lie ahead.

This effort is well worth it, as CTA will provide a huge science return in astrophysics, particle physics, cosmology and fundamental physics, and lead to a bright future for ground-based gamma ray astronomy.

Acknowledgements We gratefully acknowledge financial support from the following agencies and organisations: Ministerio de Ciencia, Tecnología e Innovación Productiva (MinCyT), Comisión Nacional de Energía Atómica (CNEA) and Consejo Nacional de Investigaciones Científicas y Técnicas (CONICET) Argentina; State Committee of Science of Armenia; Ministry for Research, CNRS-INSU, CNRS-IN2P3 and CEA, France; Max Planck Society, BMBF, DESY, Helmholtz Association, Germany; MIUR, Italy; Netherlands Research School for Astronomy (NOVA), Netherlands Organization for Scientific Research (NWO); Ministry of Science and Higher Education and the National Centre for Research and Development, Poland; MICINN support through the National $\mathrm{R}+\mathrm{D}+\mathrm{I}$, CDTI funding plans and the CPAN and MultiDark Consolider-Ingenio 2010 programme, Spain. Swedish Research Council, Royal Swedish Academy of Sciences financed, Sweden; Swiss National Science Foundation (SNSF), Switzerland; Leverhulme Trust, Royal Society, Science and Technologies Facilities Council, Durham University, UK; 
National Science Foundation, Department of Energy, Argonne National Laboratory, University of California, University of Chicago, Iowa State University, Institute for Nuclear and Particle Astrophysics (INPAC-MRPI program), Washington University McDonnell Center for the Space Sciences, USA.

Open Access This article is distributed under the terms of the Creative Commons Attribution Noncommercial License which permits any noncommercial use, distribution, and reproduction in any medium, provided the original author(s) and source are credited.

\section{References}

1. See, e.g., Aharonian, F.A.: The H.E.S.S. survey of the inner galaxy in very high energy gamma rays. Astrophys. J. 636, 777 (2006) (and subsequent presentations in conferences)

2. Barrau, A., et al.: The CAT imaging telescope for very-high-energy gamma-ray astronomy. NIMPA 416, 278 (1998)

3. See, e.g., Aharonian, F.A.: The energy spectrum of TEV gamma rays from the Crab Nebula as measured by the HEGRA system of imaging air cerenkov telescopes. Astrophys. J. 539, $317(2000)$

4. Aharonian, F.A., Völk, H.J., Horns, D. (eds.): Proc. 2nd Int. Meeting on High Energy Gamma-Ray Astronomy Heidelberg (2004). AIP Conference Proceedings, vol. 745 (2004)

5. Aharonian, F.A., Hofmann, W., Rieger, F. (eds.): Proc. 4th Int. Meeting on High Energy Gamma-Ray Astronomy, Heidelberg (2008). AIP Conference Proceedings, vol. 1085 (2008)

6. Hinton, J.A., Hofmann, W.: Teraelectronvolt astronomy. Annu. Rev. Astron. Astrophys. 47, $523(2009)$

7. Aharonian, F.A., et al.: High energy astrophysics with ground-based gamma ray detectors. Rep. Prog. Phys. 71, 096901 (2008)

8. Buckley, J., et al.: The Status and future of ground-based TeV gamma-ray astronomy. A White Paper prepared for the division of Astrophysics of the American physical society, AGIS White Paper. arXiv:0810.0444v1

9. 2nd Fermi Symposium, Washington, Capitol Hill, eConf Proceedings C091122. http://www.slac.stanford.edu/econf/C0911022/ (2009)

10. Reynolds, S.P.: Supernova remnants at high energy. Annu. Rev. Astron. Astrophys. 46, 89 (2008)

11. Malkov, M.A., Drury, L.O'C.: Nonlinear theory of diffusive acceleration of particles by shock waves. Rep. Prog. Phys. 64, 429 (2001)

12. Aharonian, F.A., Atoyan, A.M.: On the emissivity of $\pi^{0}$-decay gamma radiation in the vicinity of accelerators of galactic cosmic rays. Astron. Astrophys. 309, 917 (1996)

13. Gabici, S., Aharonian, F.A., Blasi, P.: Gamma ray signatures of ultra high energy cosmic ray accelerators: electromagnetic cascade versus synchrotron radiation of secondary electrons. Astrophys. Space Sci. 309, 365 (2007)

14. 31st ICRC, Łódź, Poland. http://icrc2009.uni.lodz.pl/ (2009)

15. Aharonian, F.A., et al.: Energy dependent $\gamma$-ray morphology in the pulsar wind nebula HESS J1825-137. Astron. Astrophys. 460, 365 (2006)

16. Dalton, M., et al.: Presentation at TeV Particle Astrophysics 2010, Paris (2010)

17. Aharonian, F.A., et al.: First detection of a VHE gamma-ray spectral maximum from a cosmic source: HESS discovery of the Vela X nebula. Astron. Astrophys. 448, L43 (2006)

18. Gaensler, B.M., Slane, P.O.: The evolution and structure of pulsar wind nebulae. Annu. Rev. Astron. Astrophys. 44, 17 (2006)

19. de Jager, O.C., Djannati-Atai, A.: In: Becker, W. (ed.) Springer Lecture Notes on "Neutron Stars and Pulsars: 40 Years After Their Discovery”. arXiv:0803.0116v1 (2008)

20. Aharonian, F.A., et al.: Very high energy gamma rays from the direction of Sagittarius A*. Astron. Astrophys. 425, L13 (2004)

21. Albert, J., et al.: Observation of gamma rays from the galactic center with the MAGIC telescope. Astrophys. J. 638 (2006) L101 638, L101 (2006) 
22. Zhang, J.L., Bi, X.J., Hu, H.B.: Very high energy $\gamma$ ray absorption by the galactic interstellar radiation field. Astron. Astrophys. 449, 641 (2006)

23. Abdo, A., et al.: Fermi LAT Observations of LS I +61'303: First Detection of an Orbital Modulation in GeV Gamma Rays. Astrophys. J. 701, L123 (2009)

24. Fender, R.: Jets from X-ray binaries. In: Lewin, W.H.G., van der Klis, M. (eds.) Compact Stellar X-Ray Sources. Cambridge University Press, Cambridge (2003)

25. Levinson, A.: High-energy aspects of astrophysical jets. Int. J. Mod. Phys. A21, 6015 (2006)

26. Butt, Y.: Beyond the myth of the supernova-remnant origin of cosmic rays. Nature 460, 701 (2009)

27. Marti, J.: Proc. High Energy Phenomena in Massive Stars. University of Jaen. ASP Conference Series, vol. 422. ISBN: 978-1-58381-724-7 (2010)

28. Aliu, E., et al.: Science 322, 1222 (2009)

29. Rea, N., Torres, D.F. (eds.): Proc. 1st Session of the Sant Cugat Forum of Astrophysics: ICREA International Workshop on The High-Energy Emission from Pulsars and Their Systems. Springer. ISSN:1570-6591 (2010)

30. Jones, T.W., O'Dell, S.L., Stein, W.A.: Physics of compact nonthermal sources. Theory of radiation rocesses. Astrophys. J. 188, 353 (1974)

31. Pian, E., et al.: BeppoSAX observations of unprecedented synchrotron activity in the BL lacertae object markarian 501. Astrophys. J. 492, L17 (1998)

32. Kino, M., Takahara, F., Kusunose, M.: Energetics of TeV blazars and physical constraints on their emission regions. Astrophys. J. 564, 97 (2002)

33. Dermer, C.D., Schlickeiser, R.: Model for the high-energy emission from blazars. Astrophys. J. 416, 458 (1993)

34. Sikora, M., Begelman, M.C., Rees, M.J.: Comptonization of diffuse ambient radiation by a relativistic jet: The source of gamma rays from blazars? Astrophys. J. 421, 153 (1994)

35. Mannheim, K., Biermann, P.L., Kruells, W.M.: A novel mechanism for nonthermal X-ray emission. Astron. Astrophys. 251, 723 (1991)

36. Aharonian, F.A.: TeV gamma rays from BL Lac objects due to synchrotron radiation of extremely high energy protons. New Astron. 5, 377 (2000)

37. See, e.g., Aharonian, F.A., et al.: Fast variability of tera-electron volt $\gamma$ rays from the radio galaxy M87. Science 314, 1424 (2006)

38. See, e.g., Miralda Escude, J.: The dark age of the universe. Science 300, 1904 (2000)

39. Romero, G.E., Aharonian, F.A., Paredes, J.M. (eds.): Proc. High-Energy Phenomena in Relativistic Outflows II, Buenos Aires, Argentina (2009); Int. J. Mod. Phys. D19, 635-1048 (2010); http://hepro2.iar-conicet.gov.ar/talks.html

40. Krolik, J.H., Pier, E.A.: Relativistic motion in gamma-ray bursts. Astrophys. J. 373, 277 (1991)

41. Fenimore, E.E., Epstein, R.I., Ho, C.: The escape of $100 \mathrm{MeV}$ photons from cosmological gamma-ray bursts. Astron. Astrophys. 97, 59 (1993)

42. Woods, E., Loeb, A.: Empirical constraints on source properties and host galaxies of cosmological gamma-ray bursts. Astrophys. J. 453, 583 (1995)

43. See, e.g., Berger, E., et al.: The afterglow and elliptical host galaxy of the short $\gamma$-ray burst GRB 050724. Nature 438, 988 (2005)

44. See, e.g., MacFadyen, A.I., Woosley, S.E.: Collapsars: Gamma-ray bursts and explosions in "failed supernovae", Astrophys. J. 524, 262 (1999)

45. See, e.g., Blasi, P., Amato, E., Caprioli, D.: The maximum momentum of particles accelerated at cosmic ray modified shocks. Mon. Not. R. Astron. Soc. 375, 1471 (2007)

46. Bertone, G. (eds.): Particle Dark Matter. Cambridge University Press, Cambridge. ISBN 13:9780521763684 (2010)

47. Adriani, O., et al.: An anomalous positron abundance in cosmic rays with energies 1.5$100 \mathrm{GeV}$. Nature 458, 607 (2009)

48. Abdo, A., et al.: Measurement of the Cosmic Ray $\mathrm{e}^{+}+\mathrm{e}^{-}$Spectrum from $20 \mathrm{GeV}$ to $1 \mathrm{TeV}$ with the Fermi Large Area Telescope. Phys. Rev. Lett. 102, 181101 (2009)

49. Abdo, A., et al.: A limit on the variation of the speed of light arising from quantum gravity effects. Nature 462, 331 (2009)

50. Martinez, M.: Fundamental physics with cosmic gamma rays. J. Phys. Conf. Ser. 171, 012013 (2009)

51. Hanbury Brown, R.: The intensity interferometer. Taylor \& Francis, London (1974) 
52. Le Bohec, S., Holder, J.: Optical intensity interferometry with atmospheric cerenkov telescope arrays. Astrophys. J. 649, 399 (2006)

53. Herbst, W., Shevchenko, K.S.: A photometric catalog of herbig AE/BE Stars and discussion of the nature and cause of the variations of UX orionis stars. Astron. J. 118, 1043 (1999)

54. Kieda, D.B., Swordy, S.P., Wakely, S.P.: A high resolution method for measuring cosmic ray composition beyond $10 \mathrm{TeV}$, Astropart. Astropart. Phys. 15, 287 (2001)

55. Aharonian, F.A., et al.: First ground-based measurement of atmospheric Cherenkov light from cosmic rays. Phys. Rev. D 75, 042004 (2007)

56. Hinton, J.: Ground-based gamma-ray astronomy with Cherenkov telescopes. New J. Phys. 11, 055005 (2009)

57. Hinton, J., et al. (H.E.S.S. Collaboration): Background modelling in ground-based Cherenkov astronomy. In: Proc. Towards a Network of Atmospheric Cherenkov Detectors VII, Palaiseau, France, pp. 183-190 (2005)

58. Albert, J., et al. (MAGIC Collaboration): Very High Energy Gamma-Ray Observations During Moonlight and Twilight with the MAGIC Telescope [astro-ph/0702475]

59. Hofmann, W.: Performance limits for Cherenkov instruments. In: Proc. Towards a Network of Atmospheric Cherenkov Detectors VII, Palaiseau, France [astro-ph/0603076] (2005)

60. Maier, G., Knapp, J.: Cosmic-ray events as background in imaging atmospheric Cherenkov telescopes. Astropart. Phys. 28, 72 [astro-ph/0704.3567] (2007)

61. Amsler, C., et al. (Particle Data Group): Phys. Lett. B 667, 1 (2008) (Section 27.5. Electromagnetic cascades, p. 276)

62. Aharonian, F.A., et al. (H.E.S.S. Collaboration): First ground-based measurement of atmospheric Cherenkov light from cosmic rays. Phys. Rev. D 75, 042004 (2007)

63. Sahakian, V., Aharonian, F., Akhperjanian, A.: Cherenkov light in electron-induced air showers. Astropart. Phys. 25, 233 (2006)

64. Aliu, E., et al.: Improving the performance of the single-dish Cherenkov telescope MAGIC through the use of signal timing. Astropart. Phys. 30, 293 (2009)

65. Schliesser, A., Mirzoyan, R.: Wide-field prime-focus imaging atmospheric Cherenkov telescopes: A systematic study. Astropart. Phys. 24, 382 (2005)

66. Vassiliev, V.V., Fegan, S.J., Brousseau, P.F.: Wide field aplanatic two-mirror telescopes for ground-based $\gamma$-ray astronomy. Astropart. Phys. 28, 10 [astro-ph/0612718] (2007)

67. Aharonian, F., et al.: On the optimization of multichannel cameras for imaging atmospheric Cherenkov telescopes. J. Phys. G 21, 985 (1995)

68. de Naurois, M., Rolland, L.: A high performance likelihood reconstruction of $\gamma$-rays for imaging atmospheric Cherenkov telescopes. Astropart. Phys. 32, 231 (2009)

69. Albert, J., et al.: FADC signal reconstruction for the MAGIC telescope. Nucl. Instrum. Methods A594, 407 (2008)

70. Mirzoyan, R., et al.: Tagging single muons and other long-flying relativistic charged particles by ultra-fast timing in air Cherenkov telescopes. Astropart. Phys. 25, 342 (2006)

71. Albert, J., et al. (MAGIC Collaboration): FADC signal reconstruction for the MAGIC telescope. Nucl. Instrum. Methods A594, 407-419 [astro-ph/0612385] (2008)

72. Funk, S., et al.: The trigger system of the H.E.S.S. telescope array. Astropart. Phys. 22, 285 [astro-ph0408375] (2004)

73. Heck, D., et al.: CORSIKA: a Monte Carlo code to simulate extensive air showers. Technical Report FZKA 6019, Forschungszentrum Karlsruhe. http://www-ik.fzk.de/corsika/ (1998)

74. Kertzman, M.P., Sembroski, G.H.: Computer simulation methods for investigating the detection characteristics of TeV air Cherenkov telescopes. Nucl. Instrum. Methods A343, 629 (1994)

75. Bernlöhr, K.: Simulation of imaging atmospheric Cherenkov telescopes with CORSIKA and sim_telarray. Astropart. Phys. 30, 149 (2008)

76. Guy, J.: Premiers résultats de l'expérience HESS et étude du potentiel de détection de matière noire supersymétrique. Thèse de doctorat, Université Paris V (2003)

77. Majumdar, P., et al.: Monte Carlo simulations for the MAGIC telescope. In: Proc. 29th ICRC, Pune, vol. 5, p. 203 (2005)

78. Hillas, M.: Cerenkov light images of EAS produced by primary gamma. Proc. 19th ICRC, La Jolla, vol. 3, p. 445 (1985) 
79. Bock, R.K., Chilingarian, A., Gaug, M., et al.: Methods for multidimensional event classification: a case study using images from a Cherenkov gamma-ray telescope. Nucl. Instrum. Methods A516, 511 (2004)

80. Ohm, S., van Eldik, C., Egberts, K.: $\gamma /$ hadron separation in very-high-energy $\gamma$-ray astronomy using a multivariate analysis method. Astropart. Phys. 31, 383 (2009)

81. Fiasson, A., Dubois, F., Masbou, J., Lamanna, G., Rosier-Lees, S.: Optimization of multivariate analysis for IACT stereoscopic systems. Astropart. Phys. 34, 25 (2010)

82. Lemoine-Goumard, M., Degrange, B., Tluczykont, M.: Selection and 3D-reconstruction of gamma-ray-induced air showers with a stereoscopic system of atmospheric Cherenkov telescopes. Astropart. Phys. 25, 195 (2006)

83. Cornils, R., et al.: The optical system of the H.E.S.S. imaging atmospheric Cherenkov telescopes. Part II: mirror alignment and point spread function. Astropart. Phys. 20, 129 (2003)

84. Aharonian, F.A., et al.: An exceptional very high energy gamma-ray flare of PKS 2155-304. Astrophys. J. 664, L71 (2007)

85. Battistoni, G., et al.: The FLUKA code: description and benchmarking. Proc. Hadronic Shower Simulation Workshop 2006, Fermilab 2006. In: Albrow, M., Raja, R. (eds.) AIP Conf. Proc., vol. 896, p. 31 (2007); Fasso, A., et al.: CERN-2005-10 (2005), INFN/TC_05/11, SLAC-R-773

86. Bass, S.A., et al.: Microscopic models for ultrarelativistic heavy ion collisions. Prog. Part. Nucl. Phys. 41, 225 (1998); Bleicher, M., et al.: Relativistic hadron-hadron collisions in the ultra-relativistic quantum molecular dynamics model. J. Phys. G: Nucl. Part. Phys. 25, 1859 (1999)

87. Kalmykov, N.N., et al.: Quark-gluon string model and EAS simulation problems at ultra-high energies. Nucl. Phys. B (Proc. Suppl.) 52B, 17 (1997)

88. Ostapchenko, S.S.: QGSJET-II: towards reliable description of very high energy hadronic interactions \& QGSJET-II: results for extensive air showers. Nucl. Phys. B (Proc. Suppl.) 151 143 and 147 (2006)

89. Ostapchenko, S.S.: Nonlinear screening effects in high energy hadronic interactions. Phys. Rev. D 74, 014026 (2006)

90. Engel, R., et al.: Air shower calculations with the new version of SIBYLL. Proc. 26th ICRC (Salt Lake City), vol. 1, p. 415 (1999)

91. Aharonian, F.A., et al.: Energy Spectrum of Cosmic-Ray Electrons at TeV Energies. Phys. Rev. Lett. 101, 261104 (2008)

92. Bernlöhr, K., et al.: MC Simulation and Layout Studies for a future Cherenkov Telescope Array. Proc. 30th ICRC, Meŕida, vol. 3, p. 1469 (2007)

93. Aharonian, F.A., et al.: 5@5 - a $5 \mathrm{GeV}$ energy threshold array of imaging atmospheric Cherenkov telescopes at $5 \mathrm{~km}$ altitude. Astropart. Phys. 15, 335 (2001)

94. Konopelko, A.: Altitude effect in Čerenkov light flashes of low energy gamma-ray-induced atmospheric showers. J. Phys. G: Nucl. Part. Phys. 30, 1835 (2004)

95. Funk, S., Hinton, J.A.: Monte-Carlo studies of the angular resolution of a future Cherenkov gamma-ray telescope. Proc. 4th Heidelberg Int. Symposium on High Energy Gamma-Ray Astronomy 2008

96. Maier, G., et al. (AGIS Collaboration): The Advanced Gamma-ray Imaging System (AGIS): Simulation Studies. Proc. 32nd ICRC, Łódź, Poland. arXiv:0907.5118v1 (2009)

97. Funk, S.: A new population of very high-energy gamma-ray sources detected with H.E.S.S. in the inner part of the Milky Way. PhD Thesis, University of Heidelberg (2005)

98. Schwarzschild, K.: Untersuchungen zur geometrischen Optik II: Theorie der Spiegeltelescope (1905); Abhandlungen der Gesellschaft der Wissenschaften in Göttingen, Bd 4, 2 (1905) 1

99. Ritchey, G.W.: The Modern Photographic Telescope and the New Astronomical Photography. Comptes Rendus 185, 1024 (1927)

100. Davies, J.M., Cotton, E.S.: Design of the quartermaster solar furnace. J. Sol. Energy Sci. Eng. 1, $16(1957)$

101. Bernlöhr, K., et al.: The optical system of the H.E.S.S. imaging atmospheric Cherenkov telescopes. Part I: layout and components of the system. Astropart. Phys. 20, 111 (2003)

102. Biland, A., et al. (MAGIC Collaboration): The active mirror control of the MAGIC Telescopes. Proc. 30th ICRC Merida, vol. 3, p. 1353 (2007) 
103. Doro, M., et al.: The reflective surface of the MAGIC telescope. Nucl. Instrum. Methods A595, 200 (2008)

104. Pareschi, G., et al.: Glass mirrors by cold slumping to cover $100 \mathrm{~m}^{2}$ of the MAGIC II Cherenkov telescope reflecting surface. Proc. SPIE 7018, 70180W (2008)

105. Holder, J., et al.: The first VERITAS telescope. Astropart. Phys. 25, 391 (2006)

106. Cogan, P., for the VERITAS Collaboration: Analysis of flash ADC data with VERITAS. arXiv:0709.4208v2

107. Punch, M., et al.: GigaHertz analogue memories in ground-based gamma-ray astronomy. AIP Conf. Proc. 515, 373 (2000)

108. Delagnes, E., et al.: A new GHz sampling ASIC for the H.E.S.S.-II front-end electronics. Nucl. Instrum. Methods A567, 21 (2006)

109. Tescaro, D., et al.: The readout system of the MAGIC-II Cherenkov telescope. arXiv:0907.0466

110. Hermann, G., et al.: A trigger and readout scheme for future Cherenkov Telescope Arrays. Proc. 4th Heidelberg Int. Symposium on High Energy Gamma-Ray Astronomy 2008. arXiv:0812.0762

111. Aliu, E., et al. (MAGIC Collaboration): Observation of pulsed $\gamma$-Rays above $25 \mathrm{GeV}$ from the Crab Pulsar with MAGIC. Science 322, 1221 (2008)

112. Aharonian, F., et al. (H.E.S.S. Collaboration): Calibration of cameras of the H.E.S.S. detector. Astrop. Phys. 22, 109 (2004)

113. Digel, S., et al. for the AGIS Collaboration: Talk at 2009 SLAC Users Organisation Meeting. http://www-group.slac.stanford.edu/sluo/2009AnnualMeeting/Talks/Tajima.pdf

114. Hanna, D., et al.: An LED-based flasher system for VERITAS. Nucl. Instrum. Methods A612, 278 (2009)

115. Papayannis, A., Fokitis, E.: Pierre Auger Observatory. Internal Note (1998) GAP 1998-018

116. U.S. Geological Survey, GTOPO30, http://eros.usgs.gov/products/elevation/gtopo30.html

117. Cinzano, P., Falchi, F., Elvidge, C.D.: The first World Atlas of the artificial night sky brightness. MNRAS 328, 689 (2001)

118. Erasmus, D.A.: An analysis of cloud cover and water vapor for the ALMA project. www.eso.org/gen-fac/pubs/astclim/espas/radioseeing/ (2002); An analysis and comparison of satellite-observed cloud cover and water vapor at Hanle, India and Yanbajing, Tibet. http://www.saao.ac.za/ erasmus/Projects/MPIHimalaya/MPIHimalaya_overview.html (2004) 


\section{The CTA Consortium}

M. Actis ${ }^{3}$, G. Agnetta ${ }^{49}$, F. Aharonian ${ }^{48}$, A. Akhperjanian ${ }^{8}$, J. Aleksić107, E. Aliu ${ }^{151}$, D. Allan ${ }^{123}$, I. Allekotte ${ }^{1}$, F. Antico ${ }^{3}$, L.A. Antonelli ${ }^{55}$, P. Antoranz ${ }^{111}$, A. Aravantinos ${ }^{47}$, T. Arlen ${ }^{141}$, H. Arnaldi ${ }^{1}$, S. Artmann ${ }^{33}$, K. Asano $^{84}$, H. Asorey ${ }^{1}$, J. Bähr ${ }^{35}$, A. Bais ${ }^{45}$, C. Baixeras ${ }^{108}$, S. Bajtlik ${ }^{95}$, D. Balis ${ }^{45}$, A. Bamba ${ }^{63}$, C. Barbier ${ }^{25}$, M. Barceló ${ }^{107}$, A. Barnacka ${ }^{95}$, J. Barnstedt ${ }^{43}$, U. Barres de Almeida ${ }^{42}$, J.A. Barrio ${ }^{112}$, S. Basso ${ }^{52}$, D. Bastieri ${ }^{58}$, C. Bauer ${ }^{41}$, J. Becerra ${ }^{102,103}$, Y. Becherini' ${ }^{26}$, K. Bechtol' ${ }^{138}$, J. Becker ${ }^{33}$, V. Beckmann ${ }^{20}$, W. Bednarek ${ }^{92}$, B. Behera ${ }^{35}$, M. Beilicke ${ }^{145}$, M. Belluso ${ }^{53}$, M. Benallou ${ }^{20}$, W. Benbow ${ }^{139}$, J. Berdugo ${ }^{104}$, K. Berger ${ }^{102,103}$, T. Bernardino ${ }^{111}$, K. Bernlöhr ${ }^{41}$, A. Biland ${ }^{120}$, S. Billotta ${ }^{53}$, T. Bird ${ }^{133}$, E. Birsin ${ }^{35,36}$, E. Bissaldi ${ }^{9}$, S. Blake ${ }^{125}$, O. Blanch ${ }^{107}$, A.A. Bobkov ${ }^{141}$, L. Bogacz ${ }^{33}$, M. Bogdan ${ }^{143}$, C. Boisson ${ }^{28}$, J. Boix ${ }^{107}$, J. Bolmont ${ }^{31}$, G. Bonanno ${ }^{53}$, A. Bonardit ${ }^{43}$, T. Bonev ${ }^{16}$, J. Borkowski ${ }^{95}$, O. Botner ${ }^{118}$, A. Bottani ${ }^{3}$, M. Bourgeat ${ }^{27}$, C. Boutonnet ${ }^{20}$, A. Bouvier ${ }^{142}$, S. Brau-Nogué ${ }^{22,23}$, I. Braun ${ }^{120}$, T. Bretz ${ }^{119}$, M.S. Briggs ${ }^{140}$, P. Brun ${ }^{21}$, L. Brunetti ${ }^{25}$, J.H. Buckley ${ }^{145}$, V. Bugaev ${ }^{145}$, R. Bühler ${ }^{138}$, T. Bulik ${ }^{94}$, G. Busetto ${ }^{58}$, S. Buson ${ }^{58}$, K. Byrum ${ }^{135}$, M. Cailles ${ }^{25}$, R. Cameron ${ }^{138}$, R. Canestrari52, S. Cantu ${ }^{52}$, E. Carmona ${ }^{104}$, A. Carosi ${ }^{55}$, J. Carr ${ }^{32}$, P.H. Carton ${ }^{21}$, M. Casiraghi ${ }^{52}$, H. Castarede ${ }^{30}$, O. Catalano ${ }^{49}$, S. Cavazzani ${ }^{51}$, S. Cazaux $^{21}$, B. Cerruti ${ }^{24}$, M. Cerruti ${ }^{28}$, P.M. Chadwick ${ }^{123}$, J. Chiang ${ }^{138}$, M. Chikawa ${ }^{69}$, M. Cieślar ${ }^{94}$, M. Ciesielska ${ }^{98}$, A. Cillis $^{2}$, C. Clerc ${ }^{26}$, P. Colin ${ }^{42}$, J. Colomé ${ }^{105}$, M. Compin ${ }^{27}$, P. Conconi ${ }^{52}$, V. Connaughton ${ }^{140}$, J. Conrad ${ }^{115}$, J.L. Contreras ${ }^{112}$, P. Coppi ${ }^{153}$, M. Corlier ${ }^{20}$, P. Corona ${ }^{31}$, O. Corpace ${ }^{21}$, D. Corti ${ }^{58}$, J. Cortina ${ }^{107}$, H. Costantini ${ }^{32}$, G. Cotter ${ }^{134}$, B. Courty ${ }^{20}$, S. Couturier ${ }^{26}$, S. Covino ${ }^{52}$, J. Croston ${ }^{133}$, G. Cusumano ${ }^{49}$, M.K. Daniel ${ }^{123}$, F. Dazzi ${ }^{61}$, A. de Angelis ${ }^{60}$, E. de Cea del Pozo ${ }^{105}$, E.M. de Gouveia Dal Pino ${ }^{12}$, O. de Jager ${ }^{101, \dagger}$, I. de la Calle Pérez ${ }^{113}$, G. De La Vega ${ }^{4,5}$, B. De Lotto $^{60}$, M. de Naurois ${ }^{26}$, E. de Oña Wilhelmini1, V. de Souza ${ }^{13}$, B. Decerprit ${ }^{35}$, C. Deil ${ }^{41}$, E. Delagnes ${ }^{21}$, G. Deleglise ${ }^{25}$, C. Delgado ${ }^{104}$, T. Dettlaff ${ }^{42}$, A. Di Paolo5, F. Di Pierro ${ }^{50}$, C. Díaz ${ }^{104}$, J. Dick ${ }^{43}$, H. Dickinson ${ }^{115}$, S.W. Digel ${ }^{138}$, D. Dimitrov ${ }^{16}$, G. Disset ${ }^{21}$, A. Djannati-Atai ${ }^{20}$, M. Doert ${ }^{37}$, W. Domainko ${ }^{41}$, D. Dorner ${ }^{121}$, M. Doro ${ }^{58,108}$, J.-L. Dournaux ${ }^{29}$, D. Dravins ${ }^{114}$, L. Drury ${ }^{48}$, F. Dubois ${ }^{112}$, R. Dubois ${ }^{138}$, G. Dubus ${ }^{24}$, C. Dufour ${ }^{20}$, D. Durand ${ }^{21}$, J. Dyks ${ }^{95}$, M. Dyrda ${ }^{96}$, E. Edy ${ }^{26}$, K. Egberts ${ }^{9}$, C. Eleftheriadis ${ }^{45}$, S. Elles ${ }^{25}$, D. Emmanoulopoulos ${ }^{133}$, R. Enomoto ${ }^{85}$, J.-P. Ernenwein ${ }^{32}$, M. Errando ${ }^{151}$, A. Etchegoyen ${ }^{4,5}$, A.D. Falcone ${ }^{147}$, K. Farakos ${ }^{47}$, C. Farnier ${ }^{121}$, S. Federici ${ }^{34,35}$, F. Feinstein ${ }^{27}$, D. Ferenc ${ }^{149}$, E. Fillin-Martino ${ }^{26}$, D. Fink ${ }^{42}$, C. Finley ${ }^{115}$, J.P. Finley ${ }^{148}$, R. Firpo ${ }^{107}$, D. Florin ${ }^{122}$, C. Föhr ${ }^{41}$, E. Fokitis ${ }^{47}$, Ll. Font ${ }^{108}$, G. Fontaine ${ }^{26}$, A. Fontana ${ }^{55}$, A. Förster ${ }^{41}$, L. Fortson ${ }^{150}$, N. Fouque ${ }^{25}$, C. Fransson ${ }^{116}$, G.W. Fraser ${ }^{126}$, L. Fresnillo ${ }^{111}$, C. Fruck ${ }^{42}$, Y. Fujita ${ }^{79}$, Y. Fukazawa ${ }^{64}$, S. Funk ${ }^{138}$, W. Gäbele ${ }^{43}$, S. Gabici ${ }^{20}$, A. Gadola ${ }^{122}$, N. Galante ${ }^{139}$, Y. Gallant ${ }^{27}$, B. García ${ }^{4,5}$, R.J. García López ${ }^{102,103}$,

${ }^{\dagger}$ deceased. 
D. Garrido ${ }^{108}$, L. Garrido ${ }^{110}$, D. Gascón ${ }^{110}$, C. Gasq ${ }^{25}$, M. Gaug ${ }^{108}$, J. Gaweda ${ }^{107}$, N. Geffroy ${ }^{25}$, C. Ghag ${ }^{129}$, A. Ghedina ${ }^{57}$, M. Ghigo ${ }^{52}$, E. Gianakaki ${ }^{45}$, S. Giarrusso ${ }^{49}$, G. Giavitto ${ }^{107}$, B. Giebels ${ }^{26}$, E. Giro ${ }^{54}$, P. Giubilato ${ }^{58}$, T. Glanzman ${ }^{138}$, J.-F. Glicenstein ${ }^{21}$, M. Gochna ${ }^{94}$, V. Golev ${ }^{15}$, M. Gómez Berisso ${ }^{1}$, A. González ${ }^{107}$, F. González ${ }^{6}$, F. Grañena ${ }^{107}$, R. Graciani ${ }^{110}$, J. Granot ${ }^{131}$, R. Gredig ${ }^{122}$, A. Green ${ }^{128}$, T. Greenshaw ${ }^{127}$, O. Grimm ${ }^{120}$, J. Grube ${ }^{146}$, M. Grudzińska ${ }^{94}$, J. Grygorczuk ${ }^{98}$, V. Guarino ${ }^{135}$, L. Guglielmi ${ }^{20}$, F. Guilloux ${ }^{21}$, S. Gunji ${ }^{87}$, G. Gyuk ${ }^{146}$, D. Hadasch ${ }^{105}$, D. Haefner ${ }^{42}$, R. Hagiwara ${ }^{87}$, J. Hahn ${ }^{41}$, A. Hallgren ${ }^{118}$, S. Hara ${ }^{88}$, M.J. Hardcastle ${ }^{131}$, T. Hassan ${ }^{112}$, T. Haubold ${ }^{42}$, M. Hauser ${ }^{40}$, M. Hayashida ${ }^{72}$, R. Heller ${ }^{35}$, G. Henri ${ }^{24}$, G. Hermann ${ }^{41}$, A. Herrero ${ }^{102,103}$, J.A. Hinton ${ }^{126}$, D. Hoffmann ${ }^{32}$, W. Hofmann ${ }^{41}$, P. Hofverberg ${ }^{41}$, D. Horns ${ }^{39}$, D. Hrupec ${ }^{17}$, H. Huan ${ }^{143}$, B. Huber ${ }^{122}$, J.-M. Huet ${ }^{29}$, G. Hughes ${ }^{35}$, K. Hultquist ${ }^{115}$, T.B. Humensky ${ }^{143}$, J.-F. Huppert ${ }^{31}$, A. Ibarra ${ }^{113}$, J.M. Illa ${ }^{107}$, J. Ingjald ${ }^{114}$, S. Inoue ${ }^{85}$, Y. Inoue ${ }^{72}, \mathrm{~K}$. Ioka ${ }^{68}$, C. Jablonski ${ }^{42}$, A. Jacholkowska ${ }^{31}$, M. Janiak ${ }^{95}$, P. Jean ${ }^{22,23}$, H. Jensen ${ }^{114}$, T. Jogler ${ }^{42}$, I. Jung ${ }^{38}$, P. Kaaret ${ }^{152}$, S. Kabuki ${ }^{82}$, J. Kakuwa ${ }^{64}$, C. Kalkuhl ${ }^{43}$, R. Kankanyan ${ }^{41}$, M. Kapala ${ }^{94}$, A. Karastergiou ${ }^{134}$, M. Karczewski ${ }^{98}$, S. Karkar ${ }^{32}$, N. Karlsson ${ }^{150}$, J. Kasperek ${ }^{99}$, H. Katagiri' ${ }^{66}$, K. Katarzyńskii ${ }^{97}$, N. Kawanaka ${ }^{68}$, B. Kędziora ${ }^{98}$, E. Kendziorra ${ }^{43}$, B. Khélifi' ${ }^{26}$, D. Kieda ${ }^{144}$, T. Kifune ${ }^{85}$, T. Kihm ${ }^{41}$, S. Klepser ${ }^{107}$, W. Kluźniak ${ }^{95}$, J. Knapp ${ }^{125}$, A.R. Knappy ${ }^{123}$, T. Kneiske ${ }^{39}$, J. Knödlseder ${ }^{22,23}$, F. Köck ${ }^{41}$, K. Kodani ${ }^{81}$, K. Kohri' ${ }^{68}$, K. Kokkotas ${ }^{45}$, N. Komin' ${ }^{25}$, A. Konopelko ${ }^{137}$, K. Kosack ${ }^{21}$, R. Kossakowski ${ }^{25}$, P. Kostka ${ }^{35}$, J. Kotuła ${ }^{96}$, G. Kowal ${ }^{12}$, J. Kozioł ${ }^{93}$, T. Krähenbühl ${ }^{120}$, J. Krause ${ }^{42}$, H. Krawczynski ${ }^{145}$, F. Krennrich ${ }^{136}$, A. Kretzschmann ${ }^{35}$, H. Kubo ${ }^{73}$, V.A. Kudryavtsev ${ }^{130}$, J. Kushida ${ }^{81}$, N. La Barbera ${ }^{49}$, V. La Parola ${ }^{49}$, G. La Rosa ${ }^{49}$, A. López ${ }^{107}$, G. Lamanna ${ }^{25}$, P. Laporte ${ }^{29}$, C. Lavalley ${ }^{27}$, T. Le Flour ${ }^{25}$, A. Le Padellec ${ }^{22,23}$, J.-P. Lenain ${ }^{121}$, L. Lessio ${ }^{54}$, B. Lieunard ${ }^{25}$, E. Lindfors ${ }^{19}$, A. Liolios ${ }^{45}$,

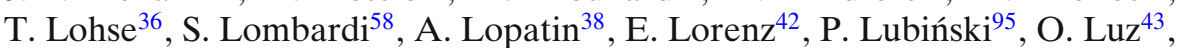
E. Lyard ${ }^{121}$, M.C. Maccarone ${ }^{49}$, T. Maccarone ${ }^{133}$, G. Maier ${ }^{35}$, P. Majumdar ${ }^{141}$, S. Maltezos ${ }^{47}$, P. Małkiewicz ${ }^{93}$, C. Mañá ${ }^{104}$, A. Manalaysay ${ }^{122}$, G. Maneva ${ }^{14}$, A. Mangano ${ }^{49}$, P. Manigot ${ }^{26}$, J. Marín ${ }^{104}$, M. Mariotti ${ }^{58}$, S. Markoff ${ }^{91}$, G. Martínez ${ }^{104}$, M. Martínez ${ }^{107}$, A. Mastichiadis ${ }^{46}$, H. Matsumoto ${ }^{77}$, S. Mattiazzo $^{58}$, D. Mazin ${ }^{107}$, T.J.L. McComb ${ }^{123}$, N. McCubbin ${ }^{132}$, I. McHardy ${ }^{133}$, C. Medina ${ }^{21}$, D. Melkumyan ${ }^{35}$, A. Mendes ${ }^{32}$, P. Mertsch ${ }^{134}$, M. Meucci ${ }^{59}$, J. Michałowski $^{96}$, P. Micolon ${ }^{21}$, T. Mineo ${ }^{49}$, N. Mirabal ${ }^{112}$,F. Mirabel ${ }^{21}$, J.M. Miranda ${ }^{111}$, R. Mirzoyan ${ }^{42}$, T. Mizuno ${ }^{64}$, B. Moal $^{26}$, R. Moderski ${ }^{95}$, E. Molinari ${ }^{57}$, I. Monteiro ${ }^{25}$, A. Moralejo ${ }^{107}$, C. Morello ${ }^{50}$, K. Mori ${ }^{75}$, G. Motta ${ }^{52}$, F. Mottez ${ }^{28}$, E. Moulin ${ }^{21}$, R. Mukherjee ${ }^{151}$, P. Munar ${ }^{109}$, H. Muraishi ${ }^{70}$, K. Murase ${ }^{85}$, A.StJ. Murphy ${ }^{129}$, S. Nagataki ${ }^{74}$, T. Naito ${ }^{88}$, T. Nakamori ${ }^{86}$, K. Nakayama ${ }^{68}$, C. Naumann ${ }^{31}$, D. Naumann ${ }^{35}$, P. Nayman ${ }^{31}$, D. Nedbal ${ }^{18}$, A. Niedźwiecki ${ }^{92}$, J. Niemiec ${ }^{96}$, A. Nikolaidis ${ }^{45}$, K. Nishijima ${ }^{81}$, S.J. Nolan ${ }^{123}$, N. Nowak ${ }^{42}$, P.T. O'Brien ${ }^{126}$, I. Ochoa ${ }^{3}$, Y. Ohira ${ }^{68}$, M. Ohishi ${ }^{85}$, H. Ohka ${ }^{85}$, A. Okumura ${ }^{67}$, C. Olivetto $^{20}$, R.A. Ong $^{141}$, R. Orito ${ }^{83}$, M. Orr ${ }^{136}$, J.P. Osborne ${ }^{126}$, M. Ostrowski ${ }^{93}$, L. Otero ${ }^{6}$, A.N. Otte ${ }^{142}$, E. Ovcharov ${ }^{15}$, I. Oya ${ }^{36}$, A. Oziębło ${ }^{100}$, S. Paiano ${ }^{58}$, J. Pallota ${ }^{6}$, J.L. Panazol ${ }^{25}$, D. Paneque ${ }^{42}$, 
M. Panter ${ }^{41}$, R. Paoletti ${ }^{59}$, G. Papyan $^{8}$, J.M. Paredes ${ }^{109}$, G. Pareschi ${ }^{52}$, R.D. Parsons ${ }^{125}$, M. Paz Arribas ${ }^{35}$, G. Pedaletti ${ }^{105}$, A. Pepato ${ }^{58}$, M. Persic ${ }^{62}$, P.O. Petrucci ${ }^{24}$, B. Peyaud ${ }^{21}$, W. Piechocki ${ }^{93}$, S. Pita ${ }^{20}$, G. Pivato ${ }^{58}$, Ł. Płatos $^{98}$, R. Platzer ${ }^{35}$, L. Pogosyan ${ }^{8}$, M. Pohl ${ }^{34,35}$, G. Pojmański ${ }^{94}$, J.D. Ponz ${ }^{113}$, W. Potter ${ }^{134}$, E. Prandini ${ }^{58}$, R. Preece ${ }^{132}$, H. Prokoph ${ }^{35}$, G. Pühlhofer ${ }^{43}$, M. Punch ${ }^{20}$, E. Quel ${ }^{6}$, A. Quirrenbach ${ }^{40}$, P. Rajda ${ }^{99}$, R. Rando $^{58}$, M. Rataj ${ }^{98}$, M. Raue ${ }^{39}$, C. Reimann ${ }^{40}$, O. Reimann ${ }^{42}$, A. Reimer ${ }^{9}$, O. Reimer ${ }^{9}$, M. Renaud ${ }^{27}$, S. Renner ${ }^{43}$, J.-M. Reymond ${ }^{21}$, W. Rhode ${ }^{37}$, M. Ribó ${ }^{109}$, M. Ribordy ${ }^{119}$, J. Rico ${ }^{106,107}$, F. Rieger ${ }^{41}$, P. Ringegni ${ }^{3}$, J. Ripken ${ }^{115}$, P. Ristori ${ }^{6}$, S. Rivoire ${ }^{27}$, L. Rob ${ }^{18}$, S. Rodriguez ${ }^{42}$, U. Roeser ${ }^{120}$, P. Romano ${ }^{49}$, G.E. Romero ${ }^{7}$, S. Rosier-Lees ${ }^{25}$, A.C. Rovero ${ }^{2}$, F. Roy ${ }^{28}$, S. Royer ${ }^{27}$, B. Rudak $^{95}$, C.B. Rulten ${ }^{123}$, J. Ruppel ${ }^{34,35}$, F. Russo ${ }^{49}$, F. Ryde ${ }^{117}$, B. Sacco $^{49}$, A. Saggion ${ }^{58}$, V. Sahakian ${ }^{8}$, K. Saito ${ }^{42}$, T. Saito ${ }^{42}$, N. Sakaki ${ }^{63}$, E. Salazar ${ }^{112}$, A. Salini ${ }^{52}$, F. Sánchez ${ }^{4,5}$, M.Á. Sánchez Conde ${ }^{102,103}$, A. Santangelo ${ }^{43}$, E.M. Santos ${ }^{11}$, A. Sanuy ${ }^{110}$, L. Sapozhnikov ${ }^{138}$, S. Sarkar ${ }^{134}$, V. Scalzotto ${ }^{58}$, V. Scapin ${ }^{60}$, M. Scarcioffolo ${ }^{58}$, T. Schanz ${ }^{43}$, S. Schlenstedt ${ }^{35}$, R. Schlickeiser ${ }^{33}$, T. Schmidt ${ }^{35}$, J. Schmoll ${ }^{124}$, M. Schroedter ${ }^{139}$, C. Schultz ${ }^{58}$, J. Schultze ${ }^{35}$, A. Schulz ${ }^{38}$, U. Schwanke ${ }^{36}$, S. Schwarzburg ${ }^{43}$, T. Schweizer ${ }^{42}$, J. Seiradakis ${ }^{45}$, S. Selmane ${ }^{20}$, K. Seweryn ${ }^{98}$, M. Shayduk ${ }^{35}$, R.C. Shellard ${ }^{10}$, T. Shibata ${ }^{63}$, M. Sikora ${ }^{95}$, J. Silk ${ }^{134}$, A. Sillanpää ${ }^{19}$, J. Sitarek ${ }^{92}$, C. Skole ${ }^{35}$, N. Smith ${ }^{137}$, D. Sobczyńska ${ }^{92}$, M. Sofo Haro ${ }^{1}$, H. Sol ${ }^{28}$, F. Spanier ${ }^{44}$, D. Spiga $^{52}$, S. Spyrou $^{26}$, V. Stamatescu ${ }^{107}$, A. Stamerra ${ }^{59}$, R.L.C. Starling ${ }^{126}$, Ł. Stawarz ${ }^{93}$, R. Steenkamp ${ }^{89}$, C. Stegmann ${ }^{38}$, S. Steiner ${ }^{122}$, N. Stergioulas ${ }^{45}$, R. Sternberger ${ }^{35}$, F. Stinzing ${ }^{38}$, M. Stodulski ${ }^{96}$, U. Straumann ${ }^{122}$, A. Suárez ${ }^{7}$, M. Suchenek ${ }^{94}$, R. Sugawara ${ }^{83}$, K.H. Sulanke ${ }^{35}$, S. Sun ${ }^{42}$, A.D. Supanitsky ${ }^{2}$, P. Sutcliffe ${ }^{127}$, M. Szanecki ${ }^{92}$, T. Szepieniec ${ }^{100}$, A. Szostek ${ }^{93}$, A. Szymkowiak ${ }^{153}$, G. Tagliaferri ${ }^{52}$, H. Tajima ${ }^{78}$, H. Takahashi ${ }^{65}$, K. Takahashi ${ }^{76}$, L. Takalo ${ }^{19}$, H. Takami ${ }^{42}$, R.G. Talbot ${ }^{124}$, P.H. Tam ${ }^{40}$, M. Tanaka ${ }^{68}$, T. Tanimori ${ }^{73}$, M. Tavani ${ }^{56}$, J.-P. Tavernet ${ }^{31}$, C. Tchernin $^{40}$, L.A. Tejedor ${ }^{112}$, I. Telezhinsky ${ }^{34,35}$, P. Temnikov ${ }^{14}$, C. Tenzer ${ }^{43}$, Y. Terada ${ }^{80}$, R. Terrier ${ }^{20}$, M. Teshima ${ }^{42,85}$, V. Testa ${ }^{55}$, L. Tibaldo ${ }^{58}$, O. Tibolla ${ }^{44}$, M. Tluczykont ${ }^{39}$, C.J. Todero Peixoto ${ }^{13}$,

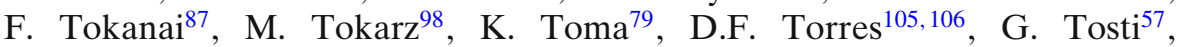
T. Totani ${ }^{72}$, F. Toussenel ${ }^{31}$, P. Vallania ${ }^{50}$, G. Vallejo ${ }^{3}$, J. van der Walt $^{101}$, C. van Eldik $^{41}$, J. Vandenbroucke ${ }^{138}$, H. Vankov ${ }^{14}$, G. Vasileiadis ${ }^{27}$, V.V. Vassiliev ${ }^{141}$, I. Vegas ${ }^{111}$, L. Venter ${ }^{28}$, S. Vercellone ${ }^{49}$, C. Veyssiere ${ }^{21}$, J.P. Vialle ${ }^{25}$, M. Videla ${ }^{4,5}$, P. Vincent ${ }^{31}$, J. Vink ${ }^{90}$, N. Vlahakis ${ }^{46}$, L. Vlahos ${ }^{45}$, P. Vogler ${ }^{120}$, A. Vollhardt ${ }^{122}$, F. Volpe ${ }^{41}$, H.P. von Gunten ${ }^{120}$, S. Vorobiov ${ }^{27}$, S. Wagner ${ }^{40}$, R.M. Wagner ${ }^{42}$, B. Wagner ${ }^{135}$, S.P. Wakely ${ }^{143}$, P. Walter ${ }^{40}$, R. Walter ${ }^{121}$, R. Warwick ${ }^{126}$, P. Wawer ${ }^{98}$, R. Wawrzaszek ${ }^{98}$, N. Webb ${ }^{22,23}$, P. Wegner ${ }^{35}$, A. Weinstein ${ }^{136}$, Q. Weitzel ${ }^{120}$, R. Welsing ${ }^{35}$, H. Wetteskind ${ }^{42}$, R. White ${ }^{126}$, A. Wierzcholska ${ }^{93}$, M.I. Wilkinson ${ }^{126}$, D.A. Williams ${ }^{142}$, M. Winde ${ }^{35}$, R. Wischnewski ${ }^{35}$, Ł. Wiśniewski ${ }^{98}$, A. Wolczko ${ }^{99}$, M. Wood $^{138}$, Q. Xiong ${ }^{137}$, T. Yamamoto ${ }^{71}$, K. Yamaoka ${ }^{63}$, R. Yamazaki ${ }^{63}$, S. Yanagita ${ }^{66}$, B. Yoffo ${ }^{20}$, M. Yonetani ${ }^{64}$, A. Yoshida ${ }^{63}$, T. Yoshida ${ }^{66}$, T. Yoshikoshi ${ }^{85}$, V. Zabalza ${ }^{109}$, A. Zagdański ${ }^{93}$, A. Zajczyk ${ }^{95}$, A. Zdziarski ${ }^{95}$, A. Zech ${ }^{28}$, K. Ziętara ${ }^{93}$, P. Ziółkowski ${ }^{96}$, V. Zitelli ${ }^{51}$, P. Zychowski ${ }^{96}$ 
${ }^{1}$ Centro Atómico Bariloche (CNEA-CONICET-IB/UNCuyo), Av. E. Bustillo 9500, (8400) San Carlos de Bariloche, Rio Negro, Argentina

${ }^{2}$ Instituto de Astronomía y Física del Espacio (CONICET-UBA), CC 67, Suc. 28, (C1428ZAA), Ciudad de Buenos Aires, Argentina

${ }^{3}$ UID GEMA - Departamento de Aeronáutica (Facultad de Ingeniera, UNLP), Calle 116 entre 47 y 48, La Plata (1900), Buenos Aires, Argentina

${ }^{4}$ Instituto de Tecnologías en Detección y Astropartículas (CNEACONICET-UNSAM), Av. Gral. Paz 1499, (1650) Buenos Aires, Argentina

${ }^{5}$ Observatorio Metereológico, Parque General San Martín (M5500ABT) Mendoza, Argentina

${ }^{6}$ CEILAP (CITEDEF-CONICET), Juan B. de La Salle 4397, (B1603ALO) Villa Martelli, Argentina.

${ }^{7}$ Instituto Argentino de Radioastronomía (CCT La Plata, CONICET), C.C.5, 1894 Villa Elisa and Facultad de Ciencias Astronómicas y Geofísicas, Universidad Nacional de La Plata, Paseo del Bosque, 1900 La Plata, Argentina

${ }^{8}$ Alikhanyan National Science Laboratory, 2 Alikhanyan Brothers St., 0036, Yerevan, Armenia

${ }^{9}$ Institut für Astro- und Teilchenphysik, Leopold-Franzens-Universität Innsbruck, 6020 Innsbruck, Austria

${ }^{10}$ Centro Brasileiro de Pesquisas Físicas, Rua Xavier Sigaud 150, CEP 22290-180, Rio de Janeiro, RJ, Brazil

${ }^{11}$ Instituto de Física, Universidade Federal do Rio de Janeiro, Av. Athos da Silveira Ramos, 149 - CT-A, CEP 21941-972, Rio de Janeiro, RJ, Brazil

${ }^{12}$ Instituto de Astronomia, Geofísico, e Ciências Atmosféricas, Universidade de São Paulo, Cidade Universitária, R. do Matão, 1226, CEP 05508-090, São Paulo, SP, Brazil

${ }^{13}$ Instituto de Física de São Carlos, Universidade de São Paulo, Av. Trabalhador são-carlense, 400 - Pq. Arnold Schimidt, CEP: 13566-590, São Carlos, SP, Brazil

${ }^{14}$ Institute for Nuclear Research and Nuclear Energy, BAS, 72 Tsarigradsko chaussee, BG-1784 Sofia, Bulgaria

${ }^{15}$ Astronomy Department, Sofia University, 5 James Bourchier Str., BG-1164 Sofia, Bulgaria

${ }^{16}$ Institute of Astronomy and NAO, BAS, 72 Tsarigradsko chaussee, BG1784 Sofia, Bulgaria

${ }^{17}$ Rudjer Boskovic Institute, Bijenicka 54, HR-10 000 Zagreb, Croatia

${ }^{18}$ Faculty of Mathematics and Physics, Institute of Particle and Nuclear Physics, Charles University, V Holešovičkách 2, 18000 Prague 8, Czech Republic

${ }^{19}$ Tuorla Observatory, University of Turku, FI-21500 Piikkiö, Finland

${ }^{20}$ APC, Bâtiment Condorcet, 10, Rue Alice Domon et Léonie Duquet, F-75205 Paris Cedex 13, France, UMR 7164 (CNRS, Université Paris 7 Denis Diderot, CEA, Observatoire de Paris)

${ }^{21}$ CEA/DSM/IRFU, CEA-Saclay, F-91191 Gif-sur-Yvette, France

${ }^{22}$ Université de Toulouse, UPS-OMP, IRAP, Toulouse, France 
${ }^{23}$ CNRS, IRAP, 9 Av. colonel Roche, BP 44346, F-31028 Toulouse cedex 4, France

${ }^{24}$ UJF-Grenoble 1 / CNRS-INSU, Institut de Planétologie et d'Astrophysique de Grenoble (IPAG) UMR 5274, Grenoble, F-38041, France

${ }^{25}$ Laboratoire d'Annecy-le-Vieux de Physique des Particules, Université de Savoie, CNRS/IN2P3, F-74941 Annecy-le-Vieux, France

${ }^{26}$ Laboratoire Leprince-Ringuet (LLR), École Polytechnique, F-91128 Palaiseau, France, UMR 7638 (CNRS, École Polytechnique)

${ }^{27}$ Laboratoire Univers et Particules de Montpellier, Université Montpellier 2, CNRS/IN2P3, CC 72, Place Eugène Bataillon, F-34095 Montpellier Cedex 5, France

${ }^{28}$ Observatoire de Paris, LUTH, CNRS, Université Paris-Diderot, 5 place Jules Janssen, 92195, Meudon, France

${ }^{29}$ Observatoire de Paris, GEPI, CNRS, Université Paris-Diderot, 5 place Jules Janssen, 92195, Meudon, France

${ }^{30}$ Observatoire de Paris, UMS, CNRS, Université Paris-Diderot, 5 place Jules Janssen, 92195, Meudon, France

${ }^{31}$ LPNHE, University of Pierre et Marie Curie, Paris 6 / University of Denis Diderot, Paris 7, CNRS/IN2P3, 4 Place Jussieu, F-75252, Paris Cedex 5, France

${ }^{32}$ CPPM, Aix-Marseille Université, CNRS/IN2P3, France

${ }^{33}$ Institut für Theoretische Physik, Lehrstuhl IV: Weltraum- und Astrophysik, Ruhr-Universität Bochum, D-44780 Bochum, Germany

${ }^{34}$ Universität Potsdam, Institut für Physik \& Astronomie, Karl-LiebknechtStrasse 24/25, D 14476 Potsdam, Germany

${ }^{35}$ DESY, Platanenallee 6, D 15738 Zeuthen, Germany

${ }^{36}$ Institut für Physik, Humboldt-Universität zu Berlin, Newtonstr. 15, D 12489 Berlin, Germany

${ }^{37}$ Department of Physics, TU Dortmund University, Otto-Hahn-Str. 4, D 44227 Dortmund

${ }^{38}$ Universität Erlangen-Nürnberg, Physikalisches Institut, Erwin-RommelStr. 1, D 91058 Erlangen, Germany

${ }^{39}$ Universität Hamburg, Institut für Experimentalphysik, Luruper Chaussee 149, D 22761 Hamburg, Germany

${ }^{40}$ Landessternwarte, Universität Heidelberg, Königstuhl, D 69117 Heidelberg, Germany

${ }^{41}$ Max-Planck-Institut für Kernphysik, P.O. Box 103980, D 69029 Heidelberg, Germany

${ }^{42}$ Max-Planck-Institut für Physik, Föhringer Ring 6, D 80805 München, Germany

${ }^{43}$ Institut für Astronomie und Astrophysik, Kepler Center for Astro and Particle Physics, Eberhard-Karls-Universität, Sand 1, D 72076 Tübingen, Germany

${ }^{44}$ Institute for Theoretical Physics and Astrophysics, Universität Würzburg, Campus Hubland Nord, Emil-Fischer-Str. 31, D-97074 Würzburg, Germany 
${ }^{45}$ Physics Department, Aristotle University of Thessaloniki, GR-54124 Thessaloniki, Greece

${ }^{46}$ Department of Astrophysics, Astronomy and Mechanics, Faculty of Physics, University of Athens, Panepistimiopolis, GR 15784 Zografos Athens, Greece

${ }^{47}$ Department of Physics, School of Applied Mathematical and Physical Sciences, National Technical University of Athens, Zografou Campus, GR-157 80 Zografou Attikis, Greece

${ }^{48}$ Dublin Institute for Advanced Studies, 31 Fitzwilliam Place, Dublin 2, Ireland

${ }^{49}$ INAF - Istituto di Astrofisica Spaziale e Fisica Cosmica di Palermo, Via U. La Malfa 153, I-90146 Palermo, Italy

${ }^{50}$ INAF - Istituto di Fisica dello Spazio Interplanetario di Torino, Corso Fiume 4, I-10133 Torino, Italy

${ }^{51}$ INAF - Osservatorio Astronomico di Bologna, via Ranzani 1, I-40127 Bologna, Italy

${ }^{52}$ INAF - Osservatorio Astronomico di Brera, Via Brera 28, 20121 Milano, Italy

${ }^{53}$ INAF - Osservatorio Astrofisico di Catania, Via S. Sofia, 78, I-95123 Catania, Italy

${ }^{54}$ INAF - Osservatorio Astronomico di Padova, Vicolo dell'Osservatorio 5, 35122 Padova, Italy

${ }^{55}$ INAF - Osservatorio Astronomico di Roma, Via Frascati 33, IĐ00040, Monteporzio Catone, Italy

${ }^{56}$ INAF - Istituto di Astrofisica Spaziale e Fisica Cosmica di Roma, Via del Fosso del Cavaliere 100, I-00133 Roma, Italy

${ }^{57}$ INAF - Telescopio Nazionale Galileo, Roque de Los Muchachos Astronomical Observatory, 38787 Garafia, TF, Spain

${ }^{58}$ Università di Padova and INFN, I-35131 Padova, Italy

${ }^{59}$ Università di Siena, and INFN Pisa, I-53100 Siena, Italy

${ }^{60}$ University of Udine and INFN Sezione di Trieste, Via delle Scienze 208 I-33100 Udine, Italy

${ }^{61}$ University of Udine and INFN Sezione di Padova, Via delle Scienze 208 I-33100 Udine, Italy

${ }^{62}$ Osservatorio Astronomico di Trieste and INFN Sezione di Trieste, Via delle Scienze 208 I-33100 Udine, Italy

${ }^{63}$ Department of Physics and Mathematics, Aoyama Gakuin University, Sagamihara, Kanagawa, 252-5258, Japan

${ }^{64}$ Department of Physical Science, Hiroshima University, Higashi-Hiroshima, Hiroshima 739-8526, Japan

${ }^{65}$ Hiroshima Astrophysical Science Center, Hiroshima University, HigashiHiroshima, 739-8526 Japan

${ }^{66}$ College of Science, Ibaraki University, Mito, Ibaraki, 310-8512, Japan

${ }^{67}$ Institute of Space and Astronautical Science, JAXA, Sagamihara, Kanagawa 252-5210, Japan 
${ }^{68}$ Institute of Particle and Nuclear Studies, KEK (High Energy Accelerator Research Organization), Tsukuba, Ibaraki 305-0801, Japan

${ }^{69}$ Dept. of Physics, Kinki University, Kowakae, Higashi-Osaka 577-8502, Japan

${ }^{70}$ School of Allied Health Sciences, Kitasato University, Kanagawa 228-8555, Japan

${ }^{71}$ Department of Physics, Konan University, Kobe, Hyogo, 658-8501 Japan

${ }^{72}$ Department of Astronomy, Graduate School of Science, Kyoto University, Sakyo-ku, Kyoto 606-8502, Japan

${ }^{73}$ Department of Physics, Graduate School of Science, Kyoto University, Sakyo-ku, Kyoto, 606-8502, Japan

${ }^{74}$ Yukawa Institute for Theoretical Physics, Kyoto University, Sakyo-ku, Kyoto 606-8502, Japan

${ }^{75}$ Department of Applied Physics, Faculty of Engineering, University of Miyazaki, Miyazaki, 889-2192, Japan

${ }^{76}$ Department of Physics and Astrophysics, Nagoya University, Chikusa-ku, Nagoya, 464-8602, Japan

${ }^{77}$ Kobayashi-Maskawa Institute (KMI) for the Origin of Particles and the Universe, Nagoya University, Chikusa-ku, Nagoya, 464-8602, Japan

${ }^{78}$ Solar-Terrestrial Environment Laboratory, Nagoya University, Chikusa-ku, Nagoya 464-8601, Japan

${ }^{79}$ Department of Earth and Space Science, Graduate School of Science, Osaka University, Toyonaka 560-0043 Japan

${ }^{80}$ Graduate School of Science and Engineering, Saitama University, Sakura-ku, Saitama City, Saitama 338-8570, Japan

${ }^{81}$ Department of Physics, Tokai University, Hiratsuka, Kanagawa 259-1292, Japan

${ }^{82}$ Tokai University Hospital, Isehara-shi, Kanagawa, 259-1193 Japan

${ }^{83}$ Institute of Socio-Arts and Sciences, The University of Tokushima, Tokushima 770-8502, Japan

${ }^{84}$ Interactive Research Center of Science, Graduate School of Science, Tokyo Institute of Technology, Meguro-ku, Tokyo 152-8550, Japan

${ }^{85}$ Institute for Cosmic Ray Research, The University of Tokyo, Kashiwa, Chiba 277-8582, Japan

${ }^{86}$ Faculty of Science and Engineering, Waseda University, Shinjuku, Tokyo 169-8555, Japan

${ }^{87}$ Yamagata University, Yamagata, Yamagata 990-8560, Japan

${ }^{88}$ Faculty of Management Information, Yamanashi Gakuin University, Kofu, Yamanashi 400-8575, Japan

${ }^{89}$ Department of Physics, University of Namibia, Private Bag 13301, Windhoek, Namibia

${ }^{90}$ Astronomical Institute, Utrecht University, P.O. Box 80000, 3508 TA, Utrecht, The Netherlands

${ }^{91}$ Astronomical Institute "Anton Pannekoek", University of Amsterdam, P.O. Box 94249, 1090 GE, Amsterdam, The Netherlands 
${ }^{92}$ Faculty of Physics and Applied Computer Science, University of Łódź, ul. Pomorska 149/153, 90-236 Łódź, Poland

${ }^{93}$ Jagiellonian University, ul. Orla 171, 30-244 Cracow, Poland

${ }^{94}$ Astronomical Observatory, University of Warsaw, Al. Ujazdowskie 4, 00-478 Warsaw, Poland

${ }^{95}$ Nicolaus Copernicus Astronomical Center, Polish Academy of Sciences, ul. Bartycka 18, 00-716 Warsaw, Poland

${ }^{96}$ Institute of Nuclear Physics, Polish Academy of Sciences, ul. Radzikowskiego 152, 31-342 Cracow, Poland

${ }^{97}$ Toruń Centre for Astronomy, Nicolaus Copernicus University, ul. Gagarina 11, 87-100 Toruń, Poland

${ }^{98}$ Space Research Centre, Polish Academy of Sciences, ul. Bartycka 18A, 00-716 Warsaw, Poland

${ }^{99}$ Faculty of Electrical Engineering, Automatics, Computer Science and Electronics, AGH University of Science and Technology, Al. Mickiewicza 30, 30-059 Cracow, Poland

${ }^{100}$ Academic Computer Centre CYFRONET AGH, ul. Nawojki 11, 30-950 Cracow, Poland

${ }^{101}$ Centre for Space Research, North-West University, Private Bag X6001, Potchefstroom, 2520 South Africa

${ }^{102}$ Instituto de Astrofísica de Canarias, E-38205 La Laguna, Tenerife, Spain

${ }^{103}$ Departamento de Astrofśica, Universidad de La Laguna, E-38206 La Laguna, Tenerife, Spain

${ }^{104}$ CIEMAT, Avda. Complutense 22, Madrid, E28040 Spain

${ }^{105}$ Institut de Ciències de l'Espai (IEEC-CSIC), Campus UAB, Torre C5, 2a planta, 08193 Barcelona, Spain

${ }^{106}$ Institució Catalana de Recerca i Estudis Avançats (ICREA)

${ }^{107}$ IFAE, Edifici Cn, Campus UAB, E-08193 Bellaterra, Spain

${ }^{108}$ Departament de Física. Universitat Autònoma de Barcelona, E-08193 Bellaterra, Spain

${ }^{109}$ Departament d'Astronomia i Meteorologia, Institut de Ciències del Cosmos (ICC), Universitat de Barcelona (IEEC-UB), Martí i Franquès, 1, 08028, Barcelona, Spain

${ }^{110}$ Departament d'Estructura i Constituents de la Matèria, Institut de Ciències del Cosmos (ICC), Universitat de Barcelona (IEEC-UB), Martí i Franquès, 1, 08028, Barcelona, Spain

${ }^{111}$ Dept. Electronics, University Complutense of Madrid, Av Complutense s/n 28040 Madrid, Spain

${ }^{112}$ Dept. FAMN. Universidad Complutense de Madrid, Grupo de Altas Energías, Av Complutense s/n 28040 Madrid, Spain

${ }^{113}$ INSA, European Space Astronomy Centre of ESA, P.O. Box 78, E-28691 Villanueva de la Cañada, Madrid, Spain

${ }^{114}$ Lund Observatory, Box 43, SE-22100 Lund, Sweden

${ }^{115}$ Oskar Klein Centre, Physics Department, Stockholm University, AlbaNova, S-106 91 Stockholm, Sweden 
${ }^{116}$ Oskar Klein Centre, Astronomy Department, Stockholm University, AlbaNova, S-106 91 Stockholm, Sweden

${ }^{117}$ Oskar Klein Centre, Department of Physics, Royal Institute of Technology (KTH), AlbaNova, S-106 91 Stockholm, Sweden

${ }^{118}$ Dept. of Physics and Astronomy, Uppsala University, Box 516, S-75120 Uppsala, Sweden

${ }^{119}$ Laboratory for High Energy Physics, École Polytechnique Fédérale, CH-1015 Lausanne, Switzerland

${ }^{120}$ ETH Zurich, Inst. for Particle Physics, Schafmattstr. 20, CH-8093 Zurich, Switzerland

${ }^{121}$ ISDC Data Centre for Astrophysics, Observatory of Geneva, University of Geneva, Chemin d'Ecogia 19, CH-1290 Versoix, Switzerland

${ }^{122}$ Physik-Institut, Universität Zürich, Winterthurerstrasse 190, 8057 Zürich, Switzerland

${ }^{123}$ Dept. of Physics, Durham University, Science Laboratories, South Road, Durham DH1 3LE, UK

${ }^{124}$ Centre for Advanced Instrumentation, Dept. of Physics, Durham University, Science Laboratories, South Road, Durham DH1 3LE, UK

${ }^{125}$ School of Physics \& Astronomy, University of Leeds, Leeds LS2 9JT, UK

${ }^{126}$ Dept. of Physics and Astronomy, University of Leicester, Leicester, LE1 7RH, UK

${ }^{127}$ University of Liverpool, Oliver Lodge Laboratory, P.O. Box 147, Oxford Street, Liverpool L69 3BX, UK

${ }^{128}$ School of Physics and Astronomy, University of Nottingham, University Park, Nottingham, NG7 2RD, UK

${ }^{129}$ School of Physics \& Astronomy, University of Edinburgh, UK

${ }^{130}$ Department of Physics and Astronomy, University of Sheffield, Hounsfield Road, Sheffield S3 7RH, UK

${ }^{131}$ Centre for Astrophysics Research, Science \& Technology Research Institute, University of Hertfordshire, Hatfield AL10 9AB, UK

${ }^{132}$ STFC Rutherford Appleton Laboratory, Harwell Oxford, Didcot OX11 OQX, UK

${ }^{133}$ School of Physics \& Astronomy, University of Southampton, Southampton SO17 1BJ, UK

${ }^{134}$ Department of Physics, University of Oxford, Keble Road, Oxford OX1 3RH, UK

${ }^{135}$ Argonne National Laboratory, 9700 S. Cass Avenue, Argonne, IL 60439, USA

${ }^{136}$ Department of Physics and Astronomy, Iowa State University, Ames, IA 50011, USA

${ }^{137}$ Department of Physics, Pittsburg State Unversity, 1701 S Broadway Str Pittsburg, KS 66762, USA

${ }^{138}$ Kavli Institute for Particle Astrophysics and Cosmology, Department of Physics and SLAC National Accelerator Laboratory, Stanford University, Stanford, CA 94305, USA 
${ }^{139}$ Harvard-Smithsonian Center for Astrophysics, 60 Garden St, MS-20, Cambridge, MA 02138, USA

${ }^{140}$ University of Alabama in Huntsville - Center for Space Physics and Aeronomic Research, 320 Sparkman Dr, Huntsville AL 35805, USA

${ }^{141}$ Department of Physics and Astronomy, University of California, Los Angeles, CA 90095, USA

${ }^{142}$ Santa Cruz Institute for Particle Physics and Department of Physics, University of California, Santa Cruz, California 95064 USA

${ }^{143}$ Enrico Fermi Institute, University of Chicago, Chicago, IL 60637, USA

${ }^{144}$ Department of Physics and Astronomy, University of Utah, Salt Lake City, UT 84112, USA

${ }^{145}$ Department of Physics, Washington University, St. Louis, MO 63130, USA

${ }^{146}$ Astronomy Department, Adler Planetarium and Astronomy Museum, Chicago, IL 60605, USA

${ }^{147}$ Dept. of Astronomy \& Astrophysics, Pennsylvania State University, University Park, PA 16802, USA

${ }^{148}$ Department of Physics, Purdue University, West Lafayette, IN 47907, USA ${ }^{149}$ University of California, Davis, One Shields Ave., Davis, CA 95616, USA

${ }^{150}$ School of Physics and Astronomy, University of Minnesota, 116 Church Street S.E., Minneapolis, Mn 55455, USA

${ }^{151}$ Barnard College, Columbia University, Dept. of Physics \& Astronomy, 3009 Broadway, New York, NY 10027

${ }^{152}$ Department of Physics and Astronomy, University of Iowa, Iowa City, IA 52242, USA

${ }^{153}$ Department of Astronomy, Yale University, P.O. Box 208101, New Haven, CT 06520-8101, USA 